\title{
THE WIENER-HOPF METHOD FOR SYSTEMS OF PSEUDODIFFERENTIAL EQUATIONS WITH AN APPLICATION TO CRACK PROBLEMS
}

\author{
R.DUDUCHAVA AND W.L.WENDLAND \\ In memoriam, dedicated to Professor Dr. V.D. Kupradze on the occasion of his \\ 90th birthday
}

\section{Contents}

INTRODUCTION 2

1. CONVOLUTIONS AND POISSON OPERATORS 6

1.1. SPACES 6

1.2. CONVOLUTION OPERATORS 9

1.3. ANISOTROPIC SPACES 10

1.4. PSEUDODIFFERENTIAL OPERATORS 11

1.5. POISSON OPERATORS 14

2. SYMBOL FACTORIZATION AND SOLVABILITY OF PsDE 19

2.1. FACTORIZATION 19

2.2. MODIFICATION OF SYMBOLS 22

2.3. SOLVABILITY RESULTS 24

3. CRACK PROBLEMS: SOLVABILITY AND REGULARITY 27

3.1. FORMULATION OF PROBLEMS 27

3.2. THE DIRICHLET PROBLEM 31

3.3. THE NEUMANN PROBLEM 35

4. CRACK PROBLEMS: ASYMPTOTICS OF SOLUTIONS 38

4.1. FORMULATION OF THEOREMS 38

4.2. PROOF OF THEOREM $4.1 \quad 40$

4.3. PROOF OF THEOREM 4.2

4.4. PROOF OF THEOREM 4.3

This work was carried out during the first author's visit in Stuttgart in 1992 and supported by the DFG priority research programme "Boundary Element Methods" within the guest-programme We-659/19-2. 
Abstract. The aim of this paper is to develop the Wiener-Hopf method for systems of pseudo-differential equations with "nonconstant coefficients" and to apply it to the describtion of the asymptotic behaviour of solutions to boundary integral equations for crack problems when a crack occurs in a linear anisotropic elastic medium. The method was suggested in [14] for scalar pseudodifferential equations with "constant coefficients" and applied in [7] to the crack problems in the anisotropic case. The existence and a-priori smoothness of such solutions has been proved in [11, 12], while the isotropic case has been treated earlier in [7, 23, 39, 47]. Our results improve even those for the isotropic case obtained in $[7,47]$. Asymptotic estimates for the behaviour of solutions in the anisotropic case have been obtained in [26] by a different method.

\section{INTRODUCTION}

The celebrated Wiener-Hopf method plays an essential rôle in investigations of pseudo-differential equations (PsDE) on the half-line $\mathbb{R}^{+}$and finds ample applications in boundary value problems (BVP) of mathematical physics for two-dimensional domains (see e.g. [10, $21,22,35,48])$. In [14] this method was adopted to the investigation of scalar multidimensional PsDEs on the half-space $\mathbb{R}_{+}^{n}$. Such a generalization gives rise to a difficult problem: the method requires the Wiener-Hopf factorization of a symbol, however, the factors of such a factorization are not in proper classes (e.g. not in Hörmander's class $S_{\gamma, \delta}^{r}\left(\mathbb{R}^{n}\right)$ ), even for $C^{\infty}$-smooth symbols, except the cases when they satisfy the celebrated transmission property (see $[4,17,18,19]$ ). The symbols which appear in problems of mathematical physics often do not have the transmission property (see e.g. Section 3 below). The arising difficulties were overcomen in [14] for $p=2$ and in $[9,41,44]$ for $1<p<\infty$. There criterions have been found for granting the Fredholm property for a PsDE on a manifold $\mathcal{M}$ with boundary $\partial \mathcal{M} \neq \emptyset$, in Bessel potential and Besov spaces, i.e. in $\mathbb{H}_{1}^{\sim}(\mathcal{M})$ and $\mathbb{B}_{1,11}^{\sim}(\mathcal{M})$, respectively. These results find many applications to BVPs of mathematical physics (see e.g. [7, 11, 12, 13, 21, 23, 39, 40, 47, 48, 51]).

The information on the existence, uniqueness and a-priori estimates of solutions which is available by the above-mentioned methods is incomplete if the boundary manifold is open as e.g. in crack problems or if it has singular submanifolds (such as conical points and edges). The solutions to such irregular BVPs are not anymore $C^{\infty}$-smooth in the vicinity of singular submanifolds; there the asymptotic behaviour of the solutions requires an additional investigation. 
The famous paper by V.Kondrat'ev [24] started the direct method of investigation of such BVPs in domains with non-smooth boundaries including cracks, and originated various interesting results (see e.g. $[6,8,16,25,26,31,32,33,34,36,37,47])$. The papers $[6,33]$ deal, for example, with the full asymptotic expansion of the solution to the Dirichlet BVP for a second order elliptic partial differential equation $(\mathrm{PDE})$ in a domain with an edge having variable opening angle $0<$ $\alpha(\omega)<2 \pi$.

Based on the results for two-dimensional elasticity obtained by M.Dauge in [8], T. von Petersdorff applied in [36] V.Kondrat'ev's technique and found the full asymptotic expansion for isotropic elastic bodies with polyhedral boundary admitting cracks and generalizing the results in [7]. V.Kondrat'ev's method for BVPs requires the solution of a corresponding Sturm-Liouville problem for some operator pencil. Such a problem for the Neumann BVP with a polygonal crack was treated by V.Kozlov and V.Maz'ya in [26] which can serve as a model problem. According to [31], this result allows to obtain some estimates of the stress singularities near to the boundary.

A different approach was suggested by G.Eskin in [14]. This procedure is based on the Wiener-Hopf method and deals with scalar PsDEs with "constant coefficients", i.e. their symbols are independent of the domain variable. Due to the explicit factorization of the symbols of some particular BVP, which seems to be rather tricky, the method was applied in $[7,47,51]$ to crack and Sommerfeld problems and to Stokes flows. For applications to two-dimensional crack and Zaremba problems see [21, 22, 48]. However, this method cannot be directly applied to the matrix and to the "variable coefficients" cases.

For demonstration of our improvements we formulate here first the main results on the asymptotic behaviour of solutions to crack problems as obtained previously by Costabel and Stephan in [7]: for the Dirichlet problem when the boundary data belong to $\mathbb{H}_{\not \neq}^{\not \nVdash / \nvdash+\sigma}(\mathbb{S})$ with $-1 / 2<$ $\sigma<1 / 2$, the solution has the form

$$
\varphi(s, \rho)=\varphi_{0}(s) \rho^{-\frac{1}{2}} \chi(\rho)+\varphi_{1}(s, \rho),
$$

where $\varphi_{0} \in \mathbb{H}_{2}^{1 / 2+\sigma}(\partial S)$ and $\varphi_{1} \in \mathbb{H}_{\not \models}^{\nVdash / \not+\sigma^{\prime}}(\mathbb{S})$ with any $\sigma^{\prime}<\sigma$, i.e. less than optimal regularity. Here $\rho$ denotes the distance to the crack front $\partial S$ and $s$ is the arc length parameter on $\partial S$. The function $\chi$ is a suitable cut-off function.

For the Neumann problem with given boundary data belonging to $\mathbb{H}_{\not \models}^{\nVdash / \not \nvdash+\sigma}(\mathbb{S})$ with $-1 / 2<\sigma<1 / 2$, the desired boundary traction can 
be represented by

$$
\psi(s, \rho)=\psi_{0}(s) \rho^{\frac{1}{2}} \chi(\rho)+\psi_{1}(s, \rho),
$$

where $\psi_{0} \in \mathbb{H}_{2}^{3 / 2+\sigma}(\partial S)$ and $\psi_{1} \in \mathbb{H}_{\not \neq}^{\not \nVdash / \not t \sigma}(\mathbb{S})$.

As already mentioned, the proof in $[7,47]$ is based on explicit factorizations of corresponding matrix symbols. In the anisotropic case, however, the explicit factorization seems to be very difficult since the symbols contain radicals ${ }^{1}$.

In our paper, however, we avoid the explicit factorizations, which in [14] was also decisive for the scalar case. Instead, we use a refined but implicit factorization result (see Lemma 2.3) based on a new version of the factorization Theorem 2.1. The latter improves a similar result in $[9,42]$. What we get is the same asymptotic representations $(0.1)$ and (0.2) but with the following improvements (cf. Theorems 4.1-4.3):

(a) the medium is anisotropic with 21 different elastic moduli in the most general case;

(b) if the boundary data in the Dirichlet problem belong to the Bessel potential space $\mathbb{H}_{p}^{1+1 / p+\sigma}(S)$ with $-1 / 2<\sigma<1 / 2$ then in (0.1) $\varphi_{0} \in \mathbb{H}_{p}^{1 / 2+\sigma}(\partial S)$ and $\varphi_{1} \in \widetilde{\mathbb{H}}_{p}^{1 / p+\sigma}(S)$. If, in particular, $p=\infty$ then $\varphi_{0} \in C^{1 / 2+\sigma^{\prime}}(\partial S)$ and $\varphi_{1} \in C^{\sigma^{\prime}}(S)$ for any $\sigma^{\prime}$ with $0<\sigma^{\prime}<\sigma$;

(c) if the boundary data in the Neumann problem belong to the Bessel potential space $\mathbb{H}_{p}^{1 / p+\sigma}(\partial S)$, then in $(0.2) \psi_{0} \in \mathbb{H}_{p}^{3 / 2+\sigma}(\partial S)$ (belonging to $C^{3 / 2+\sigma^{\prime}}(\partial S)$ if $p=\infty$ ) and $\psi_{1} \in \widetilde{\mathbb{H}}_{p}^{1+1 / p+\sigma}(S)$ (belonging to $C^{1+\sigma^{\prime}}(S)$ if $\left.p=\infty\right)$;

(d) for the canonical case when the crack coincides with the halfspace $\mathbb{R}_{+}^{2}$ and the symbol of the equation is modified, which corresponds to a lower order perturbation of the basic PsDE (cf. subsection 3.3), we find a full asymptotic expansion for the solution;

(e) asymptotic expansions similar to (0.1) and (0.2) can be obtained for general but uniquely solvable systems of PsDEs

$$
A(x, \partial) u=v \quad \text { for } \quad u \in \widetilde{\mathbb{H}}_{p}^{s}(\mathcal{M}) \quad \text { and given } \quad v \in \mathbb{H}_{p}^{s-r}(\partial \mathcal{M})
$$

on a $\mu$-smooth $n$-dimensional manifold $\mathcal{M}$ with smooth boundary $\partial \mathcal{M}$ provided that the symbol $a(x, \xi)$ belongs to the class $H_{T}^{r} C^{\mu, m}\left(\mathcal{M}, \mathbb{R}^{n}\right)$, introduced in subsection 2.1, if $1<p<\infty, \quad-\mu+1 \leq s, s-r \leq$ $\mu, \quad m>n / 2+2$.

This paper is organized as follows.

\footnotetext{
${ }^{1}$ Such an attempt has been made by M.Arzis in an unpublished paper where she succeeded only in some special cases of transversely isotropic materials.
} 
Sections 1,2 and 4 deal with the Wiener-Hopf method. Subsections 1.4, 1.5 and 2.1, 2.2 contain results important for the applications of this method. In subsection 1.4, for instance, we formulate some recent results by E.Shargorodsky on boundedness and orders of pseudodifferential operators. In subsection 1.5 we demonstrate the asymptotic expansion for Poisson operators (see Lemmata 1.12 and 1.13), which play an important rôle in Section 4. Two significant results on the factorization of symbols (see Theorem 2.1 and Lemma 2.3) and their modification (see Theorem 2.5) will be needed to derive the asymptotic representations in Section 4.

In Section 3 we demonstrate the equivalent reduction of the Dirichlet and

the

Neumann boundary value problems for an anisotropic medium to the boundary integral equations (see Theorems 3.1, 3.2 and cf. [7, 23, 27, $36,39,47])$. We also present short proofs for solvability and regularity results (see Theorems 3.3, 3.4 and cf. [11, 12]).

Finally, in Section 4, we present in Theorems 4.1-4.3 the asymptotic behaviour of solutions to crack problems. 


\section{CONVOLUTIONS AND POISSON OPERATORS}

1.1. SPACES. $\mathbb{S}\left(\mathbb{R}^{n}\right)$ denotes the Schwartz space of all fast decaying functions and $\mathbb{S}^{\prime}\left(\mathbb{R}^{n}\right)$ the dual space of tempered distributions. Since the Fourier transform and its inverse, given by

$$
\mathcal{F} \varphi(x)=\int_{\mathbb{R}^{n}} e^{i x \cdot \xi} \varphi(\xi) d \xi \quad \text { and } \quad \mathcal{F}^{-1} \psi(\xi)=\frac{1}{(2 \pi)^{n}} \int_{\mathbb{R}^{n}} e^{-i \xi \cdot x} \psi(x) d x,
$$

are bounded operators in both spaces $\mathbb{S}\left(\mathbb{R}^{n}\right)$ and $\mathbb{S}^{\prime}\left(\mathbb{R}^{n}\right)$, the convolution operator

$$
W_{a}^{0} \varphi=\mathcal{F}^{-1} a \mathcal{F} \varphi \quad \text { with } \quad a \in \mathbb{S}^{\prime}\left(\mathbb{R}^{n}\right) \quad \text { for } \quad \varphi \in \mathbb{S}\left(\mathbb{R}^{n}\right)
$$

is a bounded transformation from $\mathbb{S}\left(\mathbb{R}^{n}\right)$ into $\mathbb{S}^{\prime}\left(\mathbb{R}^{n}\right)$ (cf. $[9,13]$ ).

The Bessel potential space $\mathbb{H}_{p}^{s}\left(\mathbb{R}^{n}\right)$ is defined as a subset of $\mathbb{S}^{\prime}\left(\mathbb{R}^{n}\right)$ endowed with the norm $[49,50]$

$$
\left\|u\left|\mathbb{H}_{p}^{s}\left(\mathbb{R}^{n}\right)\|=\| \Lambda_{0}^{s} u\right| L_{p}(\mathbb{R})\right\|,
$$

where $\Lambda_{0}^{s}=W_{\lambda_{0}^{s}}^{0}$ and $\lambda_{0}^{s}(\xi)=\left(1+|\xi|^{2}\right)^{s / 2}$.

For any compact closed $\mu$-smooth $n$-dimensional manifold $\mathcal{M}(\mu \in$ $\mathbb{N}$ or $\infty)$, the space $\mathbb{H}_{1}^{\sim}(\mathcal{M})$ can be defined by a partition of the unity on $\mathcal{M}$ and local diffeomorphisms (cf. [49]) if we suppose for correctness of definition that $1-\mu \leq s \leq \mu$. For definitions of the Besov spaces $\mathbb{B}_{p, q}^{s}\left(\mathbb{R}^{n}\right)$ and $\mathbb{B}_{1,11}^{\sim}(\mathcal{M})(1 \leq p \leq \infty, 1 \leq q \leq \infty, s \in \mathbb{R}, 1-\mu \leq s \leq \mu)$ see [49]. In particular, the space $\mathbb{B}_{p, p}^{s}\left(\mathbb{R}^{n}\right)(1<p<\infty, s>0)$ coincides with all traces $\gamma_{\mathbb{R}^{n}} \varphi=\left.\varphi\right|_{\mathbb{R}^{n}}$ of functions $\varphi \in \mathbb{H}_{p}^{s+1 / p}\left(\mathbb{R}^{n+1}\right)$.

If $\mathcal{M}$ has the boundary $\partial \mathcal{M} \neq \varnothing$, then $\mathcal{M}$ can be extended to some closed manifold $\widetilde{\mathcal{M}} \supset \mathcal{M}$ of the same smoothness. The space $\widetilde{\mathbb{H}}_{p}^{s}(\mathcal{M})$ is defined as the subspace of $\mathbb{H}_{p}^{s}(\widetilde{\mathcal{M}})$ of those functions $u \in$ $\mathbb{H}_{p}^{s}(\widetilde{\mathcal{M}})$ for which $\operatorname{supp} u \subset \mathcal{M}$. Then $\mathbb{H}_{1}^{\sim}(\mathcal{M})$ denotes the quotient space $\left.\mathbb{H}_{1}^{\sim}(\mathcal{M})=\mathbb{H}_{1}^{\sim}(\widetilde{\mathcal{M}}) / \widetilde{\mathbb{H}_{1}^{\sim}} \sim \widetilde{\mathcal{M}} \mathbb{S} \lessdot \mathcal{M}\right)$ and can be identified with the space of distributions $u$ on $\mathcal{M}$ which admit an extension $l u \in \mathbb{H}_{p}^{s}(\widetilde{\mathcal{M}})$. Therefore if $r_{\mathcal{M}} \varphi=\left.\varphi\right|_{\mathcal{M}}$ denotes the restriction for $\varphi \in \mathcal{D}^{\prime}(\widetilde{\mathcal{M}})$, the tempered distributions, then $\mathbb{H}_{1}^{\sim}(\mathcal{M})$ can be identified with the space $r_{\mathcal{M}} \mathbb{H}_{p}^{s}(\widetilde{\mathcal{M}})$.

The spaces $\widetilde{\mathbb{B}}_{p, q}^{s}(\mathcal{M})$ and $\mathbb{B}_{1,11}^{\sim}(\mathcal{M})$ for $\partial \mathcal{M} \neq \varnothing$ are defined similarly $[49,50]$.

If $\mathcal{M}$ has no boundary or $\partial \mathcal{M}$ is either a special or a general Lipschitz domain (i.e. the boundary is represented locally by a Lipschitz 
function; for exact definitions see [46]) then there exist Bessel potential operators

$$
\begin{aligned}
& \Lambda_{\mathcal{M}}^{r}: \mathbb{H}_{1}^{\sim}(\mathcal{M}) \longrightarrow \mathbb{H}_{1}^{\sim-}(\mathcal{M}), \quad \widetilde{\Lambda}_{\mathcal{M}}^{r}: \widetilde{\mathbb{H}}_{p}^{s}(\mathcal{M}) \longrightarrow \widetilde{\mathbb{H}}^{s-r}(\mathcal{M}), \\
& : \mathbb{B}_{1,11}^{\sim}(\mathcal{M}) \longrightarrow \mathbb{B}_{1, \| 1}^{\sim-\backslash}(\mathcal{M}), \quad: \widetilde{\mathbb{B}}_{p, q}^{s}(\mathcal{M}) \longrightarrow \widetilde{\mathbb{B}}_{p, q}^{s-r}(\mathcal{M}), \\
& \text { where } \quad s, r \in \mathbb{R}, \quad 1<p<\infty, \quad 1 \leq q \leq \infty,
\end{aligned}
$$

which $^{2}$ define isomorphisms (see [14, 49] for a domain with smooth boundary and $[13,40]$ for a Lipschitz domain). In particular (see [14, 49] and (1.2)),

$$
\begin{array}{r}
\Lambda_{\mathbb{R}^{n}}^{r}=W_{\lambda_{0}^{r}}^{0}, \quad \Lambda_{\mathbb{R}_{-}^{n}}^{r}=W_{\lambda_{-}^{r}}^{0}, \quad \widetilde{\Lambda}_{\mathbb{R}_{+}^{n}}^{r}=W_{\lambda_{+}^{r}}^{0}, \\
\lambda_{0}^{r}(\xi)=\left(1+|\xi|^{2}\right)^{\frac{r}{2}}, \quad \lambda_{ \pm}^{r}(\xi)=\left[\xi_{n} \pm i\left(1+\left|\xi^{\prime}\right|^{2}\right)^{1 / 2}\right]^{r}, \\
r \in \mathbb{R}, \quad \xi=\left(\xi^{\prime}, \xi_{n}\right) \in \mathbb{R}^{n}, \quad \xi^{\prime} \in \mathbb{R}^{n-1}
\end{array}
$$

and moreover, $W_{\lambda_{0}^{r}}^{0}$ arranges the isometric isomorphism of spaces $\mathbb{H}_{p}^{s}\left(\mathbb{R}^{n}\right) \rightarrow$ $\mathbb{H}_{p}^{s-r}\left(\mathbb{R}^{n}\right)($ see $(1.2))$.

If $\mathbb{C}^{*}$ denotes the dual space to the space $\mathbb{C}$ and $\partial \mathcal{M} \neq \varnothing$, then the following relations are valid (see e.g. [49]):



where $\quad s \in \mathbb{R}, \quad 1<p<\infty, \quad 1 \leq q \leq \infty, \quad p^{\prime}=\frac{p}{p-1}, \quad q^{\prime}=\frac{q}{q(1.5)}$

and

$$
\begin{gathered}
\left.\left(\mathbb{H}_{1}^{\sim}(\mathcal{M})\right)^{*}=\widetilde{\mathbb{H}}_{l^{\prime}}^{-\sim}(\mathcal{M}), \quad\left(\mathbb{B}_{1,1,}^{\sim}(\mathcal{M})\right)^{*}=\widetilde{\mathbb{B}}_{l^{\prime}, l^{\prime}}^{\sim} \sim \mathcal{M}\right), \\
\text { where } \quad s \geq \frac{1}{p}, \quad 1<p<\infty, \quad 1 \leq q \leq \infty .
\end{gathered}
$$

Let $\Omega \subset \mathbb{R}^{n}$ be an $m$-dimensional $\omega$-smooth submanifold, $m<n$ and let $\gamma_{\Omega}=\left.\varphi\right|_{\Omega}$ denote the trace operator for $\varphi \in \mathbb{S}\left(\mathbb{R}^{n}\right)$. Then the operators

$$
\begin{aligned}
& \gamma_{\Omega}: \mathbb{H}_{p}^{s}\left(\mathbb{R}^{n}\right) \longrightarrow \mathbb{B}_{p, p}^{s-\frac{n-m}{p}}(\Omega), \\
&: \mathbb{B}_{p, q}^{s}\left(\mathbb{R}^{n}\right) \longrightarrow \mathbb{B}_{p, q}^{s-\frac{n-m}{p}}(\Omega), \\
& \text { for } \quad 1<p<\infty, \quad 1 \leq q \leq \infty, \quad \frac{n-m}{p}<s<\omega
\end{aligned}
$$

are bouned (see [49, Section 3.6]).

The next lemma follows from (1.7), as noted in [18, (3.20)] and in [43].

${ }^{2}$ Let us agree to ignore $\sim$ in $\widetilde{\mathbb{H}}_{p}^{s}(\mathcal{M})$ and $\quad \widetilde{\mathbb{B}}_{p, q}^{s}(\mathcal{M})$ if $\partial \mathcal{M}=\varnothing \quad$ since then the operators and spaces in (1.3) coincide. 
Lemma 1.1 (see $[18,43])$. Let $0 \leq \operatorname{dim} \Omega=m<n, \varphi \in \mathbb{B}_{p, p}^{s}(\Omega)$ $\left(\varphi \in \mathbb{B}_{p, q}^{s}(\Omega)\right)$ and $1<p<\infty(1 \leq q \leq \infty), 1-\omega+(n-m) / p^{\prime}<s<0$. Then $\varphi \otimes \delta_{\Omega} \in \mathbb{H}_{p}^{s-\frac{n-m}{p^{\prime}}}\left(\mathbb{R}^{n}\right)\left(\varphi \otimes \delta_{\Omega} \in \mathbb{B}_{p, q}^{s-\frac{n-m}{p^{\prime}}}\left(\mathbb{R}^{n}\right)\right)$, where

$$
<\varphi \otimes \delta_{\Omega}, \psi>=<\varphi, \gamma_{\Omega} \psi>\text { for } \psi \in \mathbb{S}\left(\mathbb{R}^{n}\right) .
$$

Let $X_{\nu}$ denote a parameter-dependent family of Banach spaces with $\nu \in \mathbb{R}$. If $(\cdot, \cdot)_{\theta}$ denotes the modified complex interpolation method with $0 \leq \theta \leq 1$, then the equality $\left(X_{\nu_{1}}, X_{\nu_{2}}\right)_{\theta}=X_{\theta\left(\nu_{1}, \nu_{2}\right)}$ implies that if $\bar{A}: X_{\nu_{j}} \rightarrow X_{\nu_{j+2}}$ is a bounded operator for $j=1$ and 2, then $A: X_{\theta\left(\nu_{1}, \nu_{2}\right)} \rightarrow X_{\theta\left(\nu_{3}, \nu_{4}\right)}$ is bounded for every $0 \leq \theta \leq 1$ and $\nu_{1}, \nu_{2}, \theta\left(\nu_{1}, \nu_{2}\right) \in \mathbb{R}$.

In the sequel, the following well-known interpolation properties will be applied (see [49, Sections 2.4.7, 2.5.6, 3.3.6]):

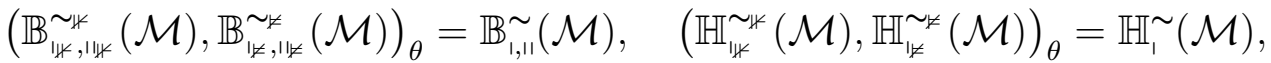

$$
\begin{aligned}
& 1<p_{j}<\infty, \quad 1<q_{j}<\infty, \quad-\frac{1}{p_{j}}<s_{j}<\infty, \quad s_{j} \in \mathbb{R}, \quad 0 \leq \theta \leq 1, \\
& s=(1-\theta) s_{1}+\theta s_{2}, \frac{1}{p}=\frac{1-\theta}{p_{1}}+\frac{\theta}{p_{2}}, \frac{1}{q}=\frac{1-\theta}{q_{1}}+\frac{\theta}{q_{2}} .
\end{aligned}
$$

We shall also use the interpolation property

$$
\left(\mathbb{H}_{\mid \nVdash}^{\sim \nVdash}(\mathcal{M}), \mathbb{H}_{\mid \nvdash}^{\sim \sharp}(\mathcal{M})\right)_{\theta, \|}=\mathbb{B}_{1, \|}^{\sim}(\mathcal{M})
$$

which can be found in $[49,2.4 .2,2.5 .6,3.3 .6]$ as well. Here the parameters have the same values as in $(1.9)$ and $(\cdot, \cdot)_{\theta, q}$ denotes the real interpolation method.

Note that (1.9) and (1.10) are also valid for the spaces $\widetilde{\mathbb{H}}_{p}^{s}(\mathcal{M})$ and $\widetilde{\mathbb{B}}_{p, q}^{s}(\mathcal{M})$ if $\partial \mathcal{M} \neq \varnothing$.

In view of (1.10), if the operator

$$
A: \mathbb{H}_{1}^{\sim}(\mathcal{M}) \rightarrow \mathbb{H}_{1}^{\sim-\backslash}(\mathcal{M})
$$

is bounded for $1<p<\infty$ and $s_{1}<s<s_{2}$, then the operator

$$
A: \mathbb{B}_{1,11}^{\sim}(\mathcal{M}) \rightarrow \mathbb{B}_{1, \| 1}^{\sim-\backslash}(\mathcal{M})
$$

will be bounded for $1<p<\infty, 1 \leq q \leq \infty$ and $s_{1}<s<s_{2}$, too.

Let now $\rho(x) \geq 0$ for $x \in \mathcal{M}$ and denote

$$
\begin{gathered}
\mathbb{H}_{p}^{s}(\mathcal{M}, \rho):=\left\{\rho^{-1} u: u \in \mathbb{H}_{1}^{\sim}(\mathcal{M})\right\}, \quad \nVdash<1<\infty, \sim \in \mathbb{R}, \\
\left\|u\left|\mathbb{H}_{p}^{s}(\mathcal{M}, \rho)\|=\| \rho u\right| \mathbb{H}_{1}^{\sim}(\mathcal{M})\right\| .
\end{gathered}
$$

If $\mathcal{M} \subset \widetilde{\mathcal{M}}$ has non-empty boundary $\partial \mathcal{M} \neq \varnothing$, then $\widetilde{\mathbb{H}}_{p}^{s}(\mathcal{M}, \rho):=$ $\left\{u \in \mathbb{H}_{p}^{s}(\widetilde{\mathcal{M}}, \rho): \operatorname{supp} u \subset \overline{\mathcal{M}} \subset \widetilde{\mathcal{M}}\right\}$. 
1.2. CONVOLUTION OPERATORS. If the convolution operator in (1.1) has the bounded extension

$$
W_{a}^{0}: L_{p}\left(\mathbb{R}^{n}\right) \rightarrow L_{p}\left(\mathbb{R}^{n}\right),
$$

we write $a \in M_{p}\left(\mathbb{R}^{n}\right)$ and $a(\xi)$ is called a (Fourier) $L_{p}$-multiplier. For $\nu \in \mathbb{R}$, let

$$
M_{p}^{(\nu)}\left(\mathbb{R}^{n}\right)=\left\{\left(1+|\xi|^{2}\right)^{\frac{\nu}{2}} a(\xi): a \in M_{p}\left(\mathbb{R}^{n}\right)\right\} .
$$

By using the isomorphisms (1.3) and (1.4) and the obvious property

$$
W_{a_{1}}^{0} W_{a_{2}}^{0}=W_{a_{1} a_{2}}^{0}, \quad a_{j} \in M_{p}^{\left(\nu_{j}\right)}\left(\mathbb{R}^{n}\right), \quad j=1,2,
$$

we get that the operator

$$
W_{a}^{0}: \mathbb{H}_{p}^{s}\left(\mathbb{R}^{n}\right) \rightarrow \mathbb{H}_{p}^{s-\nu}\left(\mathbb{R}^{n}\right)
$$

is bounded if and only if $a \in M_{p}^{(\nu)}\left(\mathbb{R}^{n}\right)$. Because of (1.10) and this inclusion, we find the boundedness of the operator

$$
W_{a}^{0}: \mathbb{B}_{p, q}^{s}\left(\mathbb{R}^{n}\right) \rightarrow \mathbb{B}_{p, q}^{s-\nu}\left(\mathbb{R}^{n}\right), \quad s \in \mathbb{R}, \quad 1<p, q<\infty .
$$

The equality $M_{2}\left(\mathbb{R}^{n}\right)=L_{\infty}\left(\mathbb{R}^{n}\right)$ is well-known. A reasonable description of the class $M_{p}^{(\nu)}\left(\mathbb{R}^{n}\right)$ for $p \neq 2$ is much more difficult and still an unsolved problem. The next theorem is known as the MikhlinHörmander-Lizorkin multiplier theorem. Proofs can be found in [41] and [20, Theorem 7.9.5].

Theorem 1.2. If

$$
\sup \left\{\left|\xi^{\beta} \partial^{\beta} a(\xi)\right|: \xi \in \mathbb{R}^{n}, \quad|\beta| \leq \frac{n+1}{2}, \quad 0 \leq \beta \leq 1\right\} \leq M<\infty,
$$

then $^{3} a \in \bigcap_{1<p<\infty} M_{p}\left(\mathbb{R}^{n}\right)$.

Let $a \in M_{p}^{(\nu)}\left(\mathbb{R}^{n}\right)$. Then the operators

Lacksquare

$$
\begin{aligned}
W_{a}:=r_{+} W_{a}^{0} & : \widetilde{\mathbb{H}}_{p}^{s}\left(\mathbb{R}_{+}^{n}\right) \rightarrow \mathbb{H}_{p}^{s-r}\left(\mathbb{R}_{+}^{n}\right), \\
& : \widetilde{\mathbb{B}}_{p, q}^{s}\left(\mathbb{R}_{+}^{n}\right) \rightarrow \mathbb{B}_{p, q}^{s-r}\left(\mathbb{R}_{+}^{n}\right),
\end{aligned}
$$

are bounded where $r_{+}:=r_{\mathbb{R}_{+}^{n}}$ is the restriction operator. This follows immediately from the above properties.

The composition rule (1.14) fails in general for half-space operators (1.16). But if there exists an analytic extension $a_{1}\left(\xi^{\prime}, \xi_{n}-i \lambda\right)$ (or

\footnotetext{
${ }^{3}$ By $0 \leq \beta \leq 1$ where $\beta=\left(\beta_{1}, \ldots, \beta_{n}\right)$ we mean $0 \leq \beta_{j} \leq 1$ for $j=1, \ldots, n$.
} 
$\left.a_{2}\left(\xi^{\prime}, \xi_{n}+i \lambda\right)\right)$ for $\xi_{n} \in \mathbb{R}$ and $\lambda \in \mathbb{R}^{+}$belonging to $\mathbb{S}^{\prime}\left(\mathbb{R}^{n-1} \times \mathbb{C}^{-}\right)$(to $\mathbb{S}^{\prime}\left(\mathbb{R}^{n-1} \times \mathbb{C}^{+}\right)$, respectively), where $\mathbb{C}^{ \pm}=\mathbb{R} \times i \mathbb{R}^{ \pm}$, then

$$
W_{a_{1}} W_{a_{2}}=W_{a_{1} a_{2}} .
$$

1.3. ANISOTROPIC SPACES. For a real constant $s \in \mathbb{R}$, we define the vectors $\bar{s}^{\prime}=(s, \ldots, s, 0)$ and $\bar{s}_{n}=(0, \ldots, 0, s)$ in $\mathbb{R}^{n}$. If $\rho(x) \geq 0$, we introduce the following anisotropic weighted Bessel potential spaces:

$$
\begin{aligned}
\mathbb{H}_{p}^{\bar{s}^{\prime}}\left(\mathbb{R}^{n}, \rho\right): & =\left\{\varphi \in \mathbb{S}^{\prime}\left(\mathbb{R}^{n}\right):\left\|\varphi \mid \mathbb{H}_{p}^{\bar{s}^{\prime}}\left(\mathbb{R}^{n}, \rho\right)\right\|\right. \\
& \left.=\left\|\rho\left(\Lambda_{\mathbb{R}^{n-1}}^{s} \otimes I\right) \varphi \mid L_{p}\left(\mathbb{R}^{n}\right)\right\|<\infty\right\}, \\
\widetilde{\mathbb{H}} \bar{s}_{p}^{\prime}\left(\mathbb{R}_{+}^{n}, \rho\right): & =\left\{\varphi \in \mathbb{H}_{p}^{\bar{s}^{\prime}}\left(\mathbb{R}^{n}, \rho\right): \operatorname{supp} \varphi \subset \overline{\mathbb{R}}_{+}^{n}\right\} .
\end{aligned}
$$

Similarly, the spaces $\mathbb{H}_{p}^{\bar{s}_{n}}\left(\mathbb{R}^{n}, \rho\right)$ and $\widetilde{\mathbb{H}}_{p}^{\bar{s}_{n}}\left(\mathbb{R}_{+}^{n}, \rho\right)$ are defined with the help of the operator $I \otimes \Lambda_{\mathbb{R}}^{s}$.

Evidently

$$
\widetilde{\mathbb{H}}_{p}^{s}\left(\mathbb{R}_{+}^{n}\right)=\widetilde{\mathbb{H}}_{p}^{s^{\prime}}\left(\mathbb{R}_{+}^{n}\right) \cap \widetilde{\mathbb{H}}_{p}^{\bar{s}_{n}}\left(\mathbb{R}_{+}^{n}\right) .
$$

Lemma 1.3. The following interpolation property is valid for weighted spaces:

$$
\begin{array}{r}
{\left[\widetilde{\mathbb{H}}_{p_{1}}^{\bar{\mu}_{n}}\left(\mathbb{R}_{+}^{n}, \rho_{1}\right), \widetilde{\mathbb{H}}_{p_{2}}^{\bar{\nu}_{n}}\left(\mathbb{R}_{+}^{n}, \rho_{2}\right)\right]_{\theta} \subset \widetilde{\mathbb{H}}_{p}^{\bar{s}_{n}}\left(\mathbb{R}_{+}^{n}, \rho\right),} \\
s=(1-\theta) \mu+\theta \nu, \quad \frac{1}{p}=\frac{1-\theta}{p_{1}}+\frac{\theta}{p_{2}}, \quad \rho=\rho_{1}^{1-\theta} \rho_{2}^{\theta}, \\
\mu, \nu \in \mathbb{R}, \quad 1<p_{1}, p_{2}<\infty, \quad 0 \leq \theta \leq 1 .
\end{array}
$$

Proof. The proof follows word by word the proof for the case of isotropic spaces with $\rho_{1}=\rho_{2}$ which is exposed in [50, Section 2.4]. For brevity we omit the details.

Lacksquare

Remark 1.4. As was proved by D.Kurtz in [28], the Mikhlin-HörmanderLizorkin theorem (see Theorem 1.2) remains valid also for weighted spaces $L_{p}\left(\mathbb{R}^{n}, \rho\right)$ if the weight function $\rho$ satisfies the Hunt-MuckenhouptWheeden condition $\rho \in A_{p}\left(\mathbb{R}^{n}\right)$.

With this result and $\rho_{1}, \rho_{2} \in A_{p}\left(\mathbb{R}^{n}\right)$ it follows that in (1.21) we also have the inverse inclusion. For a proof we refer again to [50, Section 2.4]. Then the operators $\Lambda_{\mathbb{R}^{n-1}}^{r} \otimes I$ and $I \otimes \Lambda_{+}^{r}$ represent Bessel potential operators for the anisotropic Bessel potential spaces $\mathbb{H}_{p}^{\bar{s}^{\prime}}\left(\mathbb{R}^{n}, \rho\right)$ and $\widetilde{\mathbb{H}}_{p}^{\bar{s}_{n}}\left(\mathbb{R}_{+}^{n}, \rho\right)$ respectively. 
1.4. PSEUDODIFFERENTIAL OPERATORS. If the symbol $a(x, \xi)$ depends on the external variable, then the corresponding convolution operator (see (1.1))

$$
a(x, \partial) \varphi(x)=W_{a(x, \cdot)}^{0} \varphi(x):=\left(\mathcal{F}_{\xi \rightarrow x}^{-1} a(x, \xi) \mathcal{F}_{y \rightarrow \xi} \varphi(y)\right)(x)
$$

with the symbol $a \in C\left(\mathbb{R}^{n}, \mathbb{S}^{\prime}\left(\mathbb{R}^{n}\right)\right)$ is called a general pseudo-differential operator operating on $\varphi \in \mathbb{S}\left(\mathbb{R}^{n}\right)$. Here $C(\Omega, \mathbb{C})$ denotes the set of all continuous functions $a: \Omega \rightarrow \mathbb{C}$. Let $M_{p}^{(s, s-r)}\left(\mathbb{R}^{n} \times \mathbb{R}^{n}\right)$ denote the class of symbols $a(x, \xi)$ for which the operator in (1.22) extends to the bounded mapping

$$
a(x, \partial): \mathbb{H}_{p}^{s}\left(\mathbb{R}^{n}\right) \rightarrow \mathbb{H}_{p}^{s-r}\left(\mathbb{R}^{n}\right) .
$$

The next theorem and its corollary provides us with sufficient conditions for $a \in M_{p}^{(s, s-r)}\left(\mathbb{R}^{n} \times \mathbb{R}^{n}\right)$. This theorem was proved by E.Shargorodsky in [44] with the help of a suitable parametrization. We shall use this idea in the proof of Lemma 1.7 below. Let

$$
a_{(\alpha)}(x, \xi):=\frac{\partial^{\alpha}}{\partial x^{\alpha}} a(x, \xi) \text { with } \alpha \in \mathbb{N}_{0} .
$$

Let $\Sigma^{n}$ be the class of all permutations of the single variables of $x \in$ $\mathbb{R}^{n}$

$\sigma(x)=\left(x_{(\sigma(1)}, \ldots, x_{\sigma(n)}\right)$ and

$$
a^{\sigma}(x, \xi):=a(\sigma(x), \xi) \text { for } \sigma \in \Sigma^{n} .
$$

Theorem 1.5. [44, Section 5] Let $s \in \mathbb{R}, \ell \in \mathbb{N}_{0}, 1<p<\infty$. If $a \in C\left(\mathbb{R}^{n}, M_{p}\left(\mathbb{R}^{n}\right)\right)$ and

$$
\begin{aligned}
& \max _{\sigma \in \Sigma^{n}, \alpha \in \mathbb{N}_{0}^{n},|\alpha| \leq \ell} \int_{\mathbb{R}^{m}}\left\|\frac{\partial^{m} a_{(\alpha)}^{\sigma}\left(y_{(m)}, 0, \ldots, 0, \cdot\right)}{\partial y_{(m)}} \mid M_{p}\left(\mathbb{R}^{n}\right)\right\| d y_{(m)}<\infty \\
& \left(y_{(m)}:=\left(y_{1}, \ldots, y_{m}\right)\right), \text { then } a \in M_{p}^{(s, s)}\left(\mathbb{R}^{n} \times \mathbb{R}^{n}\right) \text { for any }-\ell-1+\frac{1}{p}< \\
& s<\ell+\frac{1}{p} .
\end{aligned}
$$

Corollary 1.6. If $a \in C_{0}^{l+n}\left(\mathbb{R}^{n}, M_{p}^{(r)}\left(\mathbb{R}^{n}\right)\right.$ ) (i.e. a $(x, \xi)$ is compactly supported in $x \in \mathbb{R}^{n}$ and $\partial_{x}^{\alpha} a \in C\left(\mathbb{R}^{n}, M_{p}^{(r)}\left(\mathbb{R}^{n}\right)\right)$ for all $\left.|\alpha| \leq l+n\right)$ and $l \geq \max \{|s|,|s-r|\}$, then $a \in M_{p}^{(s, s-r)}\left(\mathbb{R}^{n} \times \mathbb{R}^{n}\right)$.

If $\left(1+|\xi|^{2}\right)^{-r / 2} \partial_{x}^{\alpha} a(x, \xi)$ satisfies condition (1.15) uniformly for all $x \in \mathbb{R}^{n}$ and $|\alpha| \leq l+n$, then $a \in \bigcap_{1<p<\infty} M_{p}^{(s, s-r)}\left(\mathbb{R}^{n} \times \mathbb{R}^{n}\right)$. 
Lemma 1.7. Let $a, b \in M_{p}^{(s, s-r)}\left(\mathbb{R}^{n} \times \mathbb{R}^{n}\right), s, r \in \mathbb{R}$. If there exist analytic extensions $a\left(x, \xi^{\prime}, \xi_{n}+i \lambda\right)$ and $b\left(x, \xi^{\prime}, \xi_{n}-i \lambda\right)$ for any $x \in \mathbb{R}^{n}$, $\xi^{\prime} \in \mathbb{R}^{n-1}, \xi_{n} \in \mathbb{R}, \lambda \in \mathbb{R}^{+}$with polynomial growth at $\infty$ (i.e. $|a|$ and $|b|$ are majorized by $\left(\left|\xi^{\prime}\right|+\left|\xi_{n}\right|+\lambda\right)^{N}$ for some $N$ and all $x \in \mathbb{R}^{n}$ uniformely), then the operators

$$
\begin{aligned}
a(x, \partial): \widetilde{\mathbb{H}}_{p}^{s}\left(\mathbb{R}_{+}^{n}\right) & \rightarrow \widetilde{\mathbb{H}}_{p}^{s-r}\left(\mathbb{R}_{+}^{n}\right) \\
r_{+} b(x, \partial) \ell: \mathbb{H}_{p}^{s}\left(\mathbb{R}_{+}^{n}\right) & \rightarrow \mathbb{H}_{p}^{s-r}\left(\mathbb{R}_{+}^{n}\right)
\end{aligned}
$$

are bounded. Here $\ell$ is any extension with $\ell \varphi \in \mathbb{H}_{p}^{s}\left(\mathbb{R}^{n}\right)$ for $\varphi \in$ $\mathbb{H}_{p}^{s}\left(\mathbb{R}_{+}^{n}\right)$. The operator in (1.25) is independent of the choice of $\ell$.

Proof. Let us apply the parametrization argument from [44]. Consider $\varphi \in \mathbb{S}\left(\mathbb{R}_{+}^{n}\right)$. Then $\mathcal{F} \varphi\left(\xi^{\prime}, \xi_{n}+i \lambda\right) \in \mathbb{S}\left(\mathbb{R}^{n-1} \times \mathbb{C}^{+}\right)$is analytic with respect to the variable $\xi_{n}+i \lambda \in \mathbb{C}^{+}$. The function $\psi\left(z, \xi^{\prime}, \xi_{n}+i \lambda\right)=$ $a\left(z, \xi^{\prime}, \xi_{n}+i \lambda\right) \mathcal{F} \varphi\left(\xi^{\prime}, \xi_{n}+i \lambda\right)$ is analytic with respect to the variable $\xi_{n}+i \lambda \in \mathbb{C}^{+}$and is fast decaying with respect to the variables $\left(\xi^{\prime}, \xi_{n}+\right.$ $i \lambda) \in \mathbb{R}^{n-1} \times \mathbb{C}^{+}$. The inverse Fourier transform $\mathcal{F}_{\xi \rightarrow x}^{-1} \psi(z, x)$ belongs then to $C^{\infty}\left(\mathbb{R}^{n}\right)$ for every $z \in \mathbb{R}^{n}$ and $\operatorname{supp} \mathcal{F}^{-1} \psi \subset \mathbb{R}^{n} \times \mathbb{R}_{+}^{n}$. Since $a(x, \partial) \varphi(x):=\mathcal{F}^{-1} \psi(x, x)$, we get $\operatorname{supp} a(x, \partial) \varphi \subset \mathbb{R}_{+}^{n}$. (1.24) follows from the fact that the subspace $\mathbb{S}\left(\mathbb{R}_{+}^{n}\right) \subset \widetilde{\mathbb{H}}_{p}^{s}\left(\mathbb{R}_{+}^{n}\right)$ is dense and the operator $a(x, \partial)$ in $(1.23)$ is continuous.

Similarly to (1.24) it follows that $r_{+} b(x, \partial) \varphi=0$ for any $\varphi \in \widetilde{\mathbb{H}}_{p}^{s}\left(\mathbb{R}_{-}^{n}\right)$ where $\mathbb{R}_{-}^{n}:=\overline{\mathbb{R}^{n} \backslash \mathbb{R}_{+}^{n}}$. If now $\ell_{1} u$ and $\ell_{2} u$ are two different extensions of $u \in \mathbb{H}_{p}^{s}\left(\mathbb{R}_{+}^{n}\right)$ in $\mathbb{H}_{p}^{s}\left(\mathbb{R}^{n}\right)$, then $\varphi=\ell_{1} u-\ell_{2} u \in \widetilde{\mathbb{H}}_{p}^{s}\left(\mathbb{R}_{-}^{n}\right)$ and therefore $r_{+} b(x, \partial) \ell_{1} u=r_{+} b(x, \partial) \ell_{2} u$. This implies

$$
\begin{gathered}
\left\|r_{+} b(x, \partial) \ell \varphi \mid \mathbb{H}_{p}^{s}\left(\mathbb{R}_{+}^{n}\right)\right\| \leq M \inf \left\{\left\|\ell \varphi \mid \mathbb{H}_{p}^{s}\left(\mathbb{R}^{n}\right)\right\|: \ell \varphi \in \mathbb{H}_{p}^{s}\left(\mathbb{R}^{n}\right)\right\} \\
=M\left\|\varphi \mid \mathbb{H}_{p}^{s}\left(\mathbb{R}_{+}^{n}\right)\right\| .
\end{gathered}
$$

$\mathbb{L}$ acksquare

For any $l, m=1,2, \ldots, \infty$ and $r \geq 0$, let $\mathbb{S}_{r}^{l, m}\left(\mathbb{R}^{n}, \mathbb{R}^{k} \times \mathbb{R}^{n-k}\right)$ denote the class of symbols $a(\xi)$ with the property

$$
\begin{array}{r}
\left|\partial_{x}^{\alpha} \partial_{\xi}^{\beta} a(x, \xi)\right| \leq M_{\alpha, \beta}(1+|\xi|)^{r-\left|\beta^{\prime \prime}\right|-\delta}\left(1+\left|\xi^{\prime}\right|\right)^{\delta-\left|\beta^{\prime}\right|}, \\
\beta=\left(\beta^{\prime}, \beta^{\prime \prime}\right), \quad \xi=\left(\xi^{\prime}, \xi^{\prime \prime}\right) \in \mathbb{R}^{n}, \quad \xi^{\prime} \in \mathbb{R}^{k}, \quad|\alpha| \leq l, \quad|\beta| \leq m,
\end{array}
$$

where $\delta=0$ if $\beta^{\prime}=0$ and $\delta=1$ if $\beta^{\prime} \neq 0$.

Lemma 1.8. Let $m>n / 2+1$ and $l \geq \max \left\{|s|,\left|s-r_{1}\right|, \mid s-\right.$ $\left.r_{2}|| s-,r_{1}-r_{2} \mid\right\}+n+1$ be integers. If $a_{j} \in \mathbb{S}_{r_{j}}^{l, m}\left(\mathbb{R}^{n}, \mathbb{R}^{k} \times \mathbb{R}^{n-k}\right)$ for $j=1,2$, then $(c f .(1.14))$

$$
a_{1}(x, \partial) a_{2}(x, \partial)=\left(a_{1} a_{2}\right)(x, \partial)+A_{-1},
$$


where $A_{-1}$ has the order $r_{1}+r_{2}-1$, i.e.

$$
A_{-1}: \mathbb{H}_{p}^{\sigma}\left(\mathbb{R}^{n}\right) \rightarrow \mathbb{H}_{p}^{\sigma-r_{1}-r_{2}+1}\left(\mathbb{R}^{n}\right)
$$

is bounded if $|\sigma|,\left|\sigma-r_{1}-r_{2}+1\right| \leq l-n-1$.

If either $a_{1}\left(x, \xi^{\prime}, \xi_{n}-i \lambda\right)$ or $a_{2}\left(x, \xi^{\prime}, \xi_{n}+i \lambda\right)$ has an analytic extension $\left(x \in \mathbb{R}^{n}, \xi^{\prime} \in \mathbb{R}^{n-1}, \xi_{n} \in \mathbb{R}, \lambda \in \mathbb{R}^{+}\right)$which is majorized by $\left(\left|\xi^{\prime}\right|+\right.$ $\left.\left|\xi_{n}\right|+\lambda\right)^{N}$ for some $N$ and for all $x \in \mathbb{R}^{n}$ uniformly, then

$$
r_{+} a_{1}(x, \partial) r_{+} a_{2}(x, \partial)=r_{+} a_{1} a_{2}(x, \partial)+A_{-1}^{+},
$$

where $A_{-1}^{+}$has the order $r_{1}+r_{2}-1$, and

$$
A_{-1}^{+}: \widetilde{\mathbb{H}}_{p}^{\sigma}\left(\mathbb{R}_{+}^{n}\right) \rightarrow \mathbb{H}_{p}^{\sigma-r_{1}-r_{2}+1}\left(\mathbb{R}_{+}^{n}\right)
$$

is bounded if $|\sigma|,\left|\sigma-r_{1}-r_{2}+1\right| \leq l-n-1$.

Proof. We have

$a_{1}(x, \partial) a_{2}(x, \partial)=b(x, \partial) \quad$ with $\quad b(x, \partial)=\left.e^{i\left(\partial_{y}, \partial_{\theta}\right)} a_{1}(x, \theta) a_{2}(y, \xi)\right|_{\theta=\xi, y=x}$ (see [20, Theorem 18.1.8]). Further, there the following estimate is shown,

$$
\begin{gathered}
\left|\partial_{x}^{\alpha} \partial_{\xi}^{\beta}\left[b(x, \xi)-a_{1}(x, \xi) a_{2}(y, \xi)\right]\right| \\
\leq C(|\alpha|,|\beta|+n / 2+3)(1+|\xi|)^{r_{1}+r_{2}-\left|\beta^{\prime \prime}\right|-1}\left(1+\left|\xi^{\prime}\right|\right)^{1-\left|\beta^{\prime}\right|}
\end{gathered}
$$

with $\beta^{\prime}, \beta^{\prime \prime}, r_{1}$ and $r_{2}$ as in (1.26), with $|\alpha| \leq l,|\beta| \leq m$ and some constant $C(M, N)$ (see $[20$, Theorems 7.6.5, 18.1.7, 18.1.8]). The first claim (1.27) follows if the second case of Corollary 1.6 is applied.

If $a_{2}\left(x, \xi^{\prime}, \xi_{n}+i \lambda\right)$ has an analytic extension with polyonomial growth, then due to (1.24) we obtain $a_{2}(x, \partial) \varphi(x)=r_{+} a_{2}(x, \partial) \varphi(x)=a_{2}(x, \partial) \varphi(x)$ for all $\varphi \in \widetilde{\mathbb{H}}_{p}^{s}\left(\mathbb{R}_{+}^{n}\right)$ and (1.28) takes the form

$$
r_{+} a_{1}(x, \partial) a_{2}(x, \partial)=r_{+}\left(a_{1} a_{2}\right)(x, \partial)+A_{-1}^{+}
$$

which follows from (1.27).

If $a_{1}\left(x, \xi^{\prime}, \xi_{n}-i \lambda\right)$ has an analytic extension with polynomial growth, we get $r_{+} a_{1}(x, \partial) r_{-}=0\left(r_{-}:=r_{\mathbb{R}_{-}^{n}}=I-r_{+}\right)$. The proof follows from the equality $r_{-} a_{1}(x, \partial) r_{-}=a_{1}(x, \partial) r_{-}$which is proved similarly to (1.24). Again we get (1.29).

Lacksquare

Corollary 1.9. Let $m>n / 2+1$, and $l \geq \max \{|s|,|s-r|+n+1\}$ be integers.

If $a \in C^{l-n}\left(\mathbb{R}^{n}\right), b \in \mathbb{S}_{r}^{l, m}\left(\mathbb{R}_{+}^{n}, \mathbb{R}^{k} \times \mathbb{R}^{n-k}\right)$ then the commutator

$$
\left[a I, r_{+} b(\cdot, \partial)\right]=a r_{+} b(\cdot, \partial)-r_{+} b(\cdot, \partial) a I
$$

has the order $r-1$, i.e. the operator

$$
[a I, b(\cdot, \partial)]: \widetilde{\mathbb{H}}_{p}^{\sigma}\left(\mathbb{R}_{+}^{n}\right) \rightarrow \mathbb{H}_{p}^{\sigma-r+1}\left(\mathbb{R}_{+}^{n}\right)
$$


is bounded if $|\sigma|,|\sigma-r| \leq l-n-1$.

1.5. POISSON OPERATORS. Let $\Omega \subset \mathbb{R}^{n}$ be some $\omega$-smooth $m$ dimensional manifold $\left(1 \leq m<n\right.$; cf. subsection 2.1), $a \in M_{p}^{(r)}\left(\mathbb{R}^{n}\right)$ and consider the following Poisson operator (cf. (1.1),(1.8))

$$
P_{\Omega}^{a} \varphi:=W_{a}^{0}\left(\varphi \otimes \delta_{\Omega}\right): C_{0}^{k}(\Omega) \rightarrow \mathbb{S}^{\prime}\left(\mathbb{R}^{n}\right) .
$$

Lemma 1.10. Let $1-k+(n-m) / p^{\prime}<s<0,-\omega+1 \leq s, r \in \mathbb{R}$, $1<p<\infty$ and $a \in M_{p}^{(r)}\left(\mathbb{R}^{n}\right)$. Then the operator

$$
P_{\Omega}^{a}: \mathbb{B}_{p, p}^{s}(\Omega) \rightarrow \mathbb{H}_{p}^{s-\frac{n-m}{p^{\prime}}-r}\left(\mathbb{R}^{n}\right)
$$

is bounded.

Proof (see $[18,44]) . \varphi \in \mathbb{B}_{p, p}^{s}(\Omega)$ implies $\varphi \otimes \delta_{\Omega} \in \mathbb{H}_{p}^{s-\frac{n-m}{p^{\prime}}}\left(\mathbb{R}^{n}\right)$ (see Lemma 1.1). Since the operator $W_{a}^{0}: \mathbb{H}_{p}^{\nu}\left(\mathbb{R}^{n}\right) \rightarrow \mathbb{H}_{p}^{\nu-r}\left(\mathbb{R}^{n}\right)$ is bounded for any $\nu \in \mathbb{R}$, there follows (1.31).

Lacksquare

Corollary 1.11. Let $\Omega=\mathbb{R}^{n-1}$ and, in addition to the conditions of Lemma 1.10 , let $a\left(\xi^{\prime}, \xi_{n}+i \lambda\right)$ have an analytic extension belonging to $\mathbb{S}^{\prime}\left(\mathbb{R}^{n-1} \times \mathbb{C}^{+}\right)$.

Then $\operatorname{supp} P_{\mathbb{R}^{n-1}}^{a} \varphi \subset \mathbb{R}_{+}^{n}$ if $\varphi \in \mathbb{B}_{p, p}^{s}\left(\mathbb{R}^{n-1}\right)$, i.e. the operator

$$
P_{\mathbb{R}^{n-1}}^{a}: \mathbb{B}_{p, p}^{s}\left(\mathbb{R}^{n-1}\right) \rightarrow \widetilde{\mathbb{H}}_{p}^{s-1 / p^{\prime}-r}\left(\mathbb{R}_{+}^{n}\right)
$$

is bounded.

Proof. The proof follows from ${ }^{4}(1.24)$ and (1.31), since $\varphi \in$ $\mathbb{B}_{p, p}^{s}\left(\mathbb{R}^{n-1}\right) \quad$ yields $\varphi \otimes \delta_{\Omega} \in \widetilde{\mathbb{H}}_{p}^{s-\frac{n-m}{p}}\left(\mathbb{R}_{+}^{n}\right) . \quad$ Lacksquare

For the asymptotic expansion in Section 4 we need the following particular Poisson operator:

$$
P_{+}^{-r} \varphi:=P_{\mathbb{R}^{n-1}}^{\widetilde{\lambda}_{+}^{-r}} \varphi, \quad \text { with } \quad \tilde{\lambda}_{+}^{-r}(\xi):=\left(\xi_{n}+i\left|\xi^{\prime}\right|+i\right)^{-r} \text { for } r>0 .
$$

Then $P_{+}^{-r}$ has an explicit representation (see [14, subsection 7.5]) as

$$
\begin{gathered}
P_{+}^{-r} \varphi(x)=c_{r} \theta_{+}\left(x_{n}\right) x_{n}^{r} e^{-x_{n}} \int_{\mathbb{R}^{n-1}} \frac{\varphi\left(y^{\prime}\right) d y^{\prime}}{\left[\left(x^{\prime}-y^{\prime}\right)^{2}+x_{n}^{2}\right]^{\frac{n}{2}}}, \\
c_{r}=\pi^{-\frac{n}{2}} e^{-i r \frac{\pi}{2}} \frac{\Gamma\left(\frac{n}{2}\right)}{\Gamma(r)}, \quad \theta_{+}\left(x_{n}\right)=\frac{1}{2}\left(1+\operatorname{sgn} x_{n}\right) .
\end{gathered}
$$

\footnotetext{
${ }^{4}$ It is easy to verify that (1.24) holds already if $a(x, \xi) \equiv a(\xi)$ and $a\left(\xi^{\prime}, \xi_{n}+i \lambda\right)$ is in $\mathbb{S}^{\prime}\left(\mathbb{R}^{n-1} \times \mathbb{C}^{+}\right)$.
} 
Let $1<p<\infty$ and define

$$
\mathcal{H}_{p}^{\infty}\left(\mathbb{R}^{n}\right):=\bigcap_{m \geq 0} \mathbb{H}_{p}^{m}\left(\mathbb{R}^{n}\right) .
$$

Due to the embeddings $\mathbb{H}_{p}^{s}\left(\mathbb{R}^{n}\right) \subset \mathbb{B}_{p, q}^{s-\varepsilon}\left(\mathbb{R}^{n}\right) \subset C^{s-2 \varepsilon-n / p}\left(\mathbb{R}^{n}\right)$ with any $\varepsilon>0$ and $s-2 \varepsilon \geq n / p$ (see [49]) we have

$$
\mathcal{H}_{p}^{\infty}\left(\mathbb{R}^{n}\right)=\bigcap_{s \geq 0} \mathbb{B}_{p, q}^{s}\left(\mathbb{R}^{n}\right) \subset\left\{\varphi \in C^{\infty}\left(\mathbb{R}^{n}\right): \varphi(\infty)=0\right\}
$$

and $\varphi \in \mathcal{H}_{p}^{\infty}\left(\mathbb{R}^{n}\right)$ implies $a(\partial) \varphi \in \mathcal{H}_{p}^{\infty}\left(\mathbb{R}^{n}\right)$ for every multiplier $a \in$ $M_{p}^{(\nu)}\left(\mathbb{R}^{n}\right)$ with any $\nu \in \mathbb{R}$.

Lemma 1.12. Let $1<p<\infty$ and $\varphi \in \mathcal{H}_{p}^{\infty}\left(\mathbb{R}^{n-1}\right)$. Then

$$
P_{+}^{-r} \varphi(x)=\theta_{+}\left(x_{n}\right) e^{-x_{n}} \sum_{k=0}^{m} c_{r, k} x_{n}^{r+k-1} \varphi_{k}\left(x^{\prime}\right)+x_{n}^{r+m} \varphi_{m+1}^{0}(x)
$$

with $\varphi_{k}=\left|\partial^{\prime}\right|^{k} \varphi \in \mathcal{H}_{p}^{\infty}\left(\mathbb{R}^{n-1}\right), c_{r, k}=\frac{(-1)^{k}}{k !} \frac{e^{-i r \frac{\pi}{2}}}{\Gamma(r)}, k=0,1, \ldots, m$, and where $\stackrel{\circ}{\varphi}_{m+1}$ satisfies $\left|\partial_{x}^{\alpha} \varphi_{m+1}^{0}(x)\right| \leq C_{\alpha} x_{n}^{-\alpha_{n}}$ for every $\alpha \in \mathbb{N}_{0}^{n}$.

Proof. Obviously, the operator $P_{+}^{-r}$ can be written as

$$
P_{+}^{-r} \varphi(x)=a\left(\partial^{\prime}, x_{n}\right) \varphi\left(x^{\prime}\right)
$$

where

$$
a\left(\xi^{\prime}, x_{n}\right)=\mathcal{F}_{\xi_{n} \rightarrow x_{n}}^{-1} \lambda_{+}^{-r}\left(\xi^{\prime}, x_{n}\right)=c_{r, 0} \theta_{+}\left(x_{n}\right) x_{n}^{r-1} e^{-x_{n}\left(1+\left|\xi^{\prime}\right|\right)}
$$

(see [14, Section 7]). Applying Taylor's expansion to $e^{-x_{n}\left|\xi^{\prime}\right| \theta}$ with respect to $\theta \in[0,1]$ and then setting $\theta=1$, we get

$$
\begin{gathered}
a\left(\xi^{\prime}, x_{n}\right)=\theta_{+}\left(x_{n}\right) e^{-x_{n}}\left[\sum_{k=0}^{m} c_{r, k} x_{n}^{r+k-1}\left|\xi^{\prime}\right|^{k}+x_{n}^{r+m} a_{m}\left(\xi^{\prime}, x_{n}\right)\left|\xi^{\prime}\right|^{m+1}\right] \\
a_{m}\left(\xi^{\prime}, x_{n}\right)=c_{r, m+1} \int_{0}^{1}(1-t)^{m} e^{-x_{n}\left|\xi^{\prime}\right| t} d t
\end{gathered}
$$

Moreover, $\varphi \in \mathcal{H}_{p}^{\infty}\left(\mathbb{R}^{n-1}\right)$ implies $\varphi_{k}=\left|\partial^{\prime}\right|^{k} \varphi \in \mathcal{H}_{p}^{\infty}\left(\mathbb{R}^{-1}\right)$ for $k=$ $0, \ldots, m+1$, (cf. (1.35)). Inserting (1.38) into (1.37) gives (1.36), where

$$
\begin{aligned}
& \varphi_{m+1}^{0}(x)=a_{m}\left(\partial^{\prime}, x_{n}\right) \varphi_{m+1}\left(x^{\prime}\right) \\
& \quad=c^{0} x_{n} \int_{\mathbb{R}^{n-1}} \varphi_{m+1}\left(y^{\prime}\right) \int_{0}^{1} \frac{t(1-t)^{m}}{\left[\left(x^{\prime}-y^{\prime}\right)^{2}+t^{2} x_{n}^{2}\right]^{\frac{n}{2}}} d t d y^{\prime} \text { for } x_{n}>0
\end{aligned}
$$


(cf. (1.33) and [14, Section 4]). From (1.39) we get

$$
\begin{aligned}
\left|\varphi_{m+1}^{0}(x)\right| & \leq c^{0} x_{n} \int_{\mathbb{R}^{n-1}}\left|\varphi_{m+1}\left(y^{\prime}\right)\right| \int_{0}^{1} \frac{t(1-t)^{m} d t}{\left[\left(x^{\prime}-y^{\prime}\right)^{2}+t^{2} x_{n}^{2}\right]^{\frac{n}{2}}} d y^{\prime} \\
& \leq c_{0}^{\prime} x_{n} \int_{0}^{1} t(1-t)^{m} \int_{\mathbb{R}^{n-1}} \frac{d y^{\prime}}{\left[\left(x^{\prime}-y^{\prime}\right)^{2}+t^{2} x_{n}^{2}\right]^{\frac{n}{2}}} d t \\
& =c_{0}^{\prime} \int_{0}^{1}(1-t)^{m} d t \int_{\mathbb{R}^{n-1}} \frac{d y^{\prime}}{\left.\left(\left|y^{\prime}\right|\right)^{2}+1\right)^{\frac{n}{2}}}=c_{0} .
\end{aligned}
$$

Since

$\partial_{x}^{\alpha} \varphi_{m+1}^{0}(x)=c^{0} \int_{\mathbb{R}^{n-1}} \partial_{y^{\prime}}^{\alpha^{\prime}} \varphi_{m+1}\left(y^{\prime}\right) \int_{0}^{1} t(1-t)^{m} \partial_{x_{n}}^{\alpha_{n}} \frac{x_{n}}{\left[\left(x^{\prime}-y^{\prime}\right)^{2}+x_{n}^{2}\right]^{\frac{n}{2}}} d t d y^{\prime}$

where $\alpha=\left(\alpha^{\prime}, \alpha_{n}\right) \in \mathbb{N}_{0}^{n}$, we get the remaining estimate in (1.36) for $\partial_{x}^{\alpha} \varphi_{m+1}^{0}$ in the same manner as in (1.40). $\quad$ Lacksquare

Lemma 1.13. Let ${ }^{5} s, r>0, \quad(1-\{r\})^{-1}<p<\infty, \quad 0<s-[r]<$ 1 and $\varphi \in \mathbb{B}_{p, p}^{s}\left(\mathbb{R}^{n-1}\right)$. Then $((1.33),(1.36))$

$$
P_{+}^{-r} \varphi(x)=c_{r} \theta_{+}\left(x_{n}\right) x_{n}^{r-1} e^{-x_{n}} \varphi\left(x^{\prime}\right)+\varphi_{1}^{0}(x) \text { with } \varphi_{1}^{0} \in \widetilde{\mathbb{H}}_{p}^{s-1 / p^{\prime}+r}\left(\mathbb{R}_{+}^{n}\right) .
$$

Proof. Obviously (cf. (1.33))

$$
\begin{aligned}
& \varphi_{1}^{0}=c_{r} \theta_{+} A_{r} \varphi \quad \text { with } \quad A_{r} \varphi(x):=x_{n}^{r} e^{-x_{n}} \int_{\mathbb{R}^{n-1}} \frac{\varphi\left(y^{\prime}\right)-\varphi\left(x^{\prime}\right)}{\left[\left(y^{\prime}-x^{\prime}\right)^{2}+x_{n}^{2}\right]^{n / 2}} d y^{\prime} \\
& =x_{n}^{r} e^{-x_{n}} \int_{\mathbb{R}^{n-1}} \frac{\Delta_{h} \varphi\left(x^{\prime}\right)}{\left[h^{2}+x_{n}^{2}\right]^{n / 2}} d h,
\end{aligned}
$$

where $\Delta_{h} \varphi\left(x^{\prime}\right)=\varphi\left(x^{\prime}+h\right)-\varphi\left(x^{\prime}\right)$. For $\psi \in C_{0}^{\infty}\left(\mathbb{R}^{n-1}\right)$ and some $\nu_{0}$, which will be chosen later, we proceed with Hölder's inequality as

\footnotetext{
${ }^{5}$ If $r>0$ then $[r] \in \mathbb{N}_{0}$ denotes the usual Gaussian bracket, i.e. the integer part of $r$ and $0 \leq\{r\}:=r-[r]<1$ and $\mathbb{N}_{0}:=\mathbb{N} \cup\{0\}$ where $\mathbb{N}$ denotes the natural integers.
} 
follows,

$$
\begin{aligned}
\left\|\left.A_{r} \psi\left|L_{p}\left(\mathbb{R}_{+}^{n}, x_{n}^{\nu_{0}}\right) \|^{p}=\int_{\mathbb{R}_{+}^{n}} x_{n}^{\left(r+\nu_{0}\right) p} e^{-x_{n} p}\right| \int_{\mathbb{R}^{n-1}} \frac{\Delta_{h} \psi\left(x^{\prime}\right)}{\left[h^{2}+x_{n}^{2}\right]^{n / 2}} d h\right|^{p} d x\right. \\
\leq \int_{\mathbb{R}_{+}^{n}} x_{n}^{\left(r+\nu_{0}\right) p}\left(\int_{\mathbb{R}^{n-1}} \frac{|h|^{\delta p^{\prime}} d h}{\left[h^{2}+x_{n}^{2}\right]^{n / 2}}\right)^{\frac{p}{p^{\prime}}} \int_{\mathbb{R}^{n-1}} \frac{\left|\Delta_{h} \psi\left(x^{\prime}\right)\right|^{p}}{|h|^{\delta p}\left[h^{2}+x_{n}^{2}\right]^{n / 2}} d h d x \\
=\int_{\mathbb{R}_{+}^{n}} x_{n}^{\left(r+\nu_{0}+\delta-1 / p^{\prime}\right) p}\left(\int_{\mathbb{R}^{n-1}} \frac{|t|^{\delta p^{\prime}} d t}{\left[t^{2}+1\right]^{n / 2}}\right)^{\frac{p}{p^{\prime}}} \int_{\mathbb{R}^{n-1}} \frac{\left|\Delta_{h} \psi\left(x^{\prime}\right)\right|^{p}}{|h|^{\delta p}\left(h^{2}+x_{n}^{2}\right)^{n / 2}} d h d x \\
=c_{1} \int_{\mathbb{R}^{n-1} \times \mathbb{R}^{n-1}} \frac{\left|\Delta_{h} \psi\left(x^{\prime}\right)\right|^{p} d x^{\prime}}{|h|^{\delta p}} \int_{0}^{\infty} \frac{x_{n}^{\left(r+\nu_{0}+\delta-1 / p^{\prime}\right) p}}{\left[h^{2}+x_{n}^{2}\right]^{n / 2}} d x_{n} d h \\
=c_{1} \int_{\mathbb{R}^{n-1} \times \mathbb{R}^{n-1}} \frac{\left|\Delta_{h} \psi\left(x^{\prime}\right)\right|^{p}}{h^{n-1+\left(1 / p^{\prime}-r-\nu_{0}\right) p}} d h d x^{\prime} \int_{0}^{\infty} \frac{t^{\left(r+\nu_{0}+\delta-1 / p^{\prime}\right) p}}{\left[1+t^{2}\right]^{n / 2}} d t \\
=c_{2}^{p}\left\|\psi \mid \mathbb{B}_{p, p}^{1 / p^{\prime}-r-\nu_{0}}\left(\mathbb{R}^{n-1}\right)\right\|^{p},
\end{aligned}
$$

(see $\left[49\right.$, p.190] for the norm in $\mathbb{B}_{p, p}^{\nu}\left(\mathbb{R}^{n-1}\right)=W_{p}^{\nu}\left(\mathbb{R}^{n-1}\right)$ ) provided

$$
-1<\delta p^{\prime}<1, \quad-1<\left(r+\nu_{0}-\delta-\frac{1}{p^{\prime}}\right) p<1, \quad 0<\frac{1}{p^{\prime}}-r-\nu_{0}<1 .
$$

The last inequality in (1.44) restricts the exponent of the weight function $t^{\nu_{0}}$ as

$$
-\frac{1}{p}<r+\nu_{0}<1-\frac{1}{p}
$$

while the two other inequalities should be satisfied by an appropriate choice of $\delta$. This is always possible since (1.44) can be rewritten as follows:

$$
1-p<\delta p<p-1, \quad \mu-1<\delta p<\mu+1, \quad 0<\mu<p,
$$

where $\mu=\left(1 / p^{\prime}-r-\nu_{0}\right) p$. The last inequality in (1.46) corresponds to (1.45), while the first two inequalities can always be satisfied since

$$
\max \{1-p, \mu-1\}<\min \{p-1, \mu+1\},
$$

if the last inequality in (1.48) holds. Thus, the operator

$$
A_{r}: \mathbb{B}_{p, p}^{1 / p^{\prime}-r-\nu_{0}}\left(\mathbb{R}^{n-1}\right) \rightarrow L_{p}\left(\mathbb{R}_{+}^{n}, x_{n}^{\nu_{0}}\right)
$$


is bounded if (1.45) is satisfied. Because of (1.42) we have

$$
\Lambda_{\mathbb{R}^{n-1}}^{\nu} A_{r} \psi=A_{r} \Lambda_{\mathbb{R}^{n-1}}^{\nu} \psi \quad \text { for } \quad \nu \in \mathbb{R} .
$$

Therefore, with (1.3), the operator

$$
A_{r}: \mathbb{B}_{p, p}^{1 / p^{\prime}-\{r\}-\nu}\left(\mathbb{R}^{n-1}\right) \rightarrow \widetilde{\mathbb{H}}_{p}^{(\nu, 0)}\left(\mathbb{R}_{+}^{n}\right)
$$

is bounded, provided $1 / p^{\prime}-\{r\}>0, \quad \nu \in \mathbb{R}$. From (1.42),(1.47) it follows that

$$
\begin{array}{r}
\left\|\partial_{x_{n}}^{k} A_{r} \psi\left|L_{p}\left(\mathbb{R}_{+}^{n}, x_{n}^{\nu_{0}+l}\right)\left\|\leq c_{3}\right\| e^{x_{n}} \partial_{x_{n}}^{k} A_{r} \psi\right| L_{p}\left(\mathbb{R}_{+}^{n}, x_{n}^{\nu_{0}+k}\right)\right\| \\
\leq c_{4}\left\|\psi \mid \mathbb{B}_{p p}^{1 / p^{\prime}-r-\nu_{0}}\left(\mathbb{R}^{n-1}\right)\right\|,
\end{array}
$$

provided $0 \leq k \leq l$ and $l=0,1, \ldots,[r]+1$. Hence,

$$
A_{r}: \mathbb{B}_{p, p}^{1 / p^{\prime}-r-\nu_{0}}\left(\mathbb{R}^{n-1}\right) \rightarrow \widetilde{\mathbb{H}}_{p}^{(0,[r]+1)}\left(\mathbb{R}_{+}^{n}, x_{n}^{\nu_{0}+[r]+1}\right)
$$

is bounded if (1.45) is satisfied.

Applying interpolation (1.21) to (1.47) and (1.50) yields that the operator

$$
\begin{aligned}
A_{r}: \mathbb{B}_{p, p}^{s}\left(\mathbb{R}^{n-1}\right) \subset \mathbb{B}_{p, p}^{1 / p^{\prime}-r-\nu_{0}}\left(\mathbb{R}^{n-1}\right) \rightarrow \widetilde{\mathbb{H}}_{p}^{(0, \nu)}\left(\mathbb{R}_{+}^{n}, x_{n}^{\nu_{0}+\nu}\right), \\
\quad \text { for } \quad 0<\frac{1}{p^{\prime}}-r-\nu_{0} \leq s, \quad 0<\nu<[r]+1,
\end{aligned}
$$

is bounded if (1.45) is satisfied. If now $\nu=-\nu_{0}=s-1 / p^{\prime}+\{r\}$, then (1.49), (1.51) and (1.20) imply the boundedness of the operator

$$
A_{r}: \mathbb{B}_{p, p}^{s}\left(\mathbb{R}^{n-1}\right) \rightarrow \widetilde{\mathbb{H}}_{p}^{s-1 / p^{\prime}+r}\left(\mathbb{R}_{+}^{n}\right)
$$

provided $1 / p^{\prime}-\{r\}>0$. Obviously the latter condition is equivalent to $1 /(1-\{r\})<p<\infty$; and from (1.45) with $\nu_{0}=-s+1 / p^{\prime}-\{r\}$ we find the condition $0<s-[r]<1$.

Lacksquare

Remark 1.14. From (1.48) and (1.42) it is evident that $\varphi_{1}^{0}\left(x^{\prime}, x_{n}\right)$ cannot be smoother with respect to the variable $x^{\prime}$ than $\varphi\left(x^{\prime}\right)$. Therefore, the condition $1 /(1-\{r\})<p<\infty$ is necessary for the asymptotic representation (1.41) to hold (see (1.52)). 


\section{SYMBOL FACTORIZATION AND SOLVABILITY OF PsDE}

2.1. FACTORIZATION. Let $\mu, m=0,1, \ldots, \infty, \quad r \in \mathbb{C}, \quad \Omega \subset$ $\mathbb{R}^{n}$. By $H^{r} C^{\mu, m}\left(\Omega, \mathbb{R}^{n}\right):=C^{\mu}\left(\Omega, H^{r} C^{m}\left(\mathbb{R}^{n}\right)\right)$ we denote the algebra of $N \times N$ matrix-functions $a(x, \xi)$ which are $\mu$-smooth with respect to the variable $x \in \bar{\Omega}=\Omega \cup \partial \Omega$, are $m$-smooth with respect to $\xi \in S^{n-1}=$ $\left\{\xi \in \mathbb{R}^{n}:|\xi|=1\right\}$ and positive homogeneous of order $r$ in $\xi$, i.e.

$$
a(x, \lambda \xi)=\lambda^{r} a(x, \xi) \text { for } \lambda>0, \quad x \in \Omega, \quad \xi \in \mathbb{R}^{n} .
$$

By $H_{T}^{r} C^{\mu, m}\left(\Omega, \mathbb{R}^{n}\right) \subset H^{r} C^{\mu, m}\left(\Omega, \mathbb{R}^{n}\right)$ we denote the subalgebra of functions $a(x, \xi)=a_{0}\left(x, t \omega^{\prime}, \xi_{n}\right)$ where $\omega^{\prime}=\left|\xi^{\prime}\right|^{-1} \xi^{\prime} \in S^{n-2}, t=\left|\xi^{\prime}\right| \in \mathbb{R}^{+}$, $\xi_{n} \in \mathbb{R}$, which meet the following conditions ${ }^{6}$

$$
\begin{aligned}
& \lim _{t \rightarrow 0} \partial_{t}^{k} a_{0}\left(x, t \omega^{\prime},-1\right)=(-1)^{k} \lim _{t \rightarrow 0} \partial_{t}^{k} a_{0}\left(x, t \omega^{\prime}, 1\right), \\
& \text { for all } \quad x \in \bar{\Omega}, \quad \omega^{\prime} \in S^{n-2}, \quad k=0,1, \ldots, m-1 .
\end{aligned}
$$

Theorem 2.1. Let $a \in H_{T}^{r} C^{\mu, m}\left(\Omega, \mathbb{R}^{n}\right)(\mu \geq 0, m \geq 1, r \in \mathbb{R})$ be $a$ positive definite $N \times N$ matrix-function satisfying

$$
(a(x, \xi) \eta, \eta) \geq M|\xi|^{r}|\eta|^{2} \quad \text { for all } \quad x \in \bar{\Omega}, \quad \xi \in \mathbb{R}^{n} \quad \text { and } \quad \eta \in \mathbb{C}^{N}
$$

with some constant $M>0$. Then $a(x, \xi)$ admits the factorization

$$
a(x, \xi)=a_{-}(x, \xi) a_{+}(x, \xi), \quad a_{ \pm}(x, \xi)=\left(\xi_{n} \pm i\left|\xi^{\prime}\right|\right)^{\frac{r}{2}} b_{ \pm}(x, \xi),
$$

where $b_{+}^{ \pm 1}\left(x, \xi^{\prime}, \xi_{n}+i \lambda\right)$ and $b_{-}^{ \pm 1}\left(x, \xi^{\prime}, \xi_{n}-i \lambda\right)$ have uniformly bounded analytic extensions for $\lambda>0, x \in \bar{\Omega}, \xi^{\prime} \in \mathbb{R}^{n-1}, \xi_{n} \in \mathbb{R}$. Moreover $b_{ \pm} \in H^{0} C^{\mu, m-1}\left(\Omega, \mathbb{R}^{n}\right)$ and

$$
\left|\partial_{x}^{\alpha} \partial_{\xi}^{\beta} b_{ \pm}^{ \pm 1}(x, \xi)\right| \leq M_{\alpha, \beta}|\xi|^{r-\beta_{n}-\delta}\left|\xi^{\prime}\right|^{\delta-\left|\beta^{\prime}\right|}
$$

for any choice of multiindices $|\alpha| \leq \mu$ and $|\beta| \leq m-1$. Here $\delta=0$ if $\beta^{\prime}=0$ and $\delta=1$ if $\beta^{\prime} \neq 0$.

Proof. Let $\Gamma_{0}=\{\zeta \in \mathbb{C}:|\zeta|=1\}$ denote the complex unit circle and let $C^{\mu, m-1}\left(\Omega, \mathbb{R}^{+} \times S^{n-2} \times \Gamma_{0}\right)=C^{\mu}\left(\Omega, C^{m-1}\left(\mathbb{R}^{+} \times S^{n-2} \times \Gamma_{0}\right)\right)$ be the algebra of functions $d_{0}\left(x, t, \omega^{\prime}, \zeta\right)$ which have continuous derivatives up to the orders $\mu$ and $(m-1)$ with respect to the variables $x \in \bar{\Omega}$ and $\left(t, \omega^{\prime}, \zeta\right) \in \mathbb{R}^{+} \times S^{n-2} \times \Gamma_{0}$, respectively. The function $b(x, \xi)=$

\footnotetext{
${ }^{6}$ If $r=0, m=\infty$, then conditions (2.1) coincide with the classical transmission property (cf. $[4,14,18,19,37])$.
} 
$|\xi|^{-r} a(x, \xi)$ is homogeneous of order 0 with respect to the variable $\xi$. Let us verify that $\widetilde{b} \in C^{\mu, m-1}\left(\Omega, \mathbb{R}^{+} \times S^{n-2} \times \Gamma_{0}\right)$ where

$$
\widetilde{b}\left(x, t, \omega^{\prime}, \zeta\right):=b\left(x, t \omega^{\prime}, i \frac{1-\zeta}{1+\zeta} t\right)=\widetilde{b}_{0}\left(x, \omega^{\prime}, \tan \frac{\theta}{2}\right),
$$

with $\omega^{\prime}=\left|\xi^{\prime}\right|^{-1} \xi^{\prime} \in S^{n-2}, \quad t=\left|\xi^{\prime}\right|, \quad \xi=\left(\xi^{\prime}, \xi_{n}\right) \in \mathbb{R}^{n}, \quad \zeta=e^{i \theta}$,

$$
i \frac{1-\zeta}{1+\zeta}=i \frac{1-e^{i \theta}}{1+e^{i \theta}}=\tan \frac{\theta}{2} \quad \text { for } \quad-\pi<\theta<\pi .
$$

For showing (2.5) it suffices to prove that all derivatives $\widetilde{b}^{(\alpha, 0, \beta, k)}\left(x, \omega^{\prime}, e^{i \theta}\right)$ defined via

$$
g^{(\alpha, l, \beta, k)}\left(x, t, \omega^{\prime}, \theta\right):=\partial_{x}^{\alpha} \partial_{t}^{l} \partial_{\omega^{\prime}}^{\beta} \partial_{\theta}^{k} g\left(x, t, \omega^{\prime}, \theta\right),
$$

are continuous at $\theta= \pm \pi$.

The continuity condition (2.1) and homogeneity lead to

$$
\begin{aligned}
\widetilde{b}^{(\alpha, 0, \beta, k)}\left(x, \omega^{\prime}, e^{i \theta}\right) & =\partial_{\theta}^{k} \widetilde{b}_{0}^{(\alpha, 0, \beta, 0)}\left(x, \pm \cot \frac{\theta}{2} \omega^{\prime}, \pm 1\right) \\
& =\sum_{j, l, m=1}^{k}( \pm)^{j} a_{j l m}(\theta) \widetilde{b}_{0}^{(\alpha, j, \beta, 0)}\left(x, \pm \cot \frac{\theta}{2} \omega^{\prime}, \pm 1\right) \\
& =\sum_{j, l, m=1}^{k} a_{j l m}(\theta) \widetilde{b}_{0}^{(\alpha, j, \beta, 0)}\left(x, \pm \cot \frac{\theta}{2} \omega^{\prime}, 1\right) \\
& \text { for } \quad 0 \leq \pm \theta \leq \pi \text { and } k \in \mathbb{N}
\end{aligned}
$$

where $a_{j l m}(\theta):=c_{j l m} \sin ^{-2 l}(\theta / 2) \cot ^{m}(\theta / 2)$, and, therefore $a_{j l m}(-\pi)=$ $a_{j l m}(\pi)$. From these relations together with $(2.1)$ we obtain the continuity properties

$$
\lim _{\theta \rightarrow-\pi} \widetilde{b}^{(\alpha, 0, \beta, k)}\left(x, \omega^{\prime}, e^{i \theta}\right)=\lim _{\theta \rightarrow \pi} \widetilde{b}^{(\alpha, 0, \beta, k)}\left(x, \omega^{\prime}, e^{i \theta}\right) .
$$

Hence, $\widetilde{b} \in C^{\mu, \nu}\left(\Omega, S^{n-2} \times \Gamma_{0}\right)=\cap_{k=0}^{m-1} C^{\mu, k}\left(\Omega, S^{n-2}, C^{\nu-k}\left(\Gamma_{0}\right)\right)$, where $C^{\sigma}\left(\Gamma_{0}\right)$ denotes the Hölder-Zygmund space and where $m-1<\nu<m$ (see [46]). A matrix-function from the algebra $C^{\sigma}\left(\Gamma_{0}\right)(\sigma>0)$ admits a factorization (see, e.g., [5]):

$$
\widetilde{b}\left(x, \omega^{\prime}, \zeta\right)=\widetilde{b}_{-}\left(x, \omega^{\prime}, \zeta\right) \widetilde{b}_{+}\left(x, \omega^{\prime}, \zeta\right),
$$

where $\widetilde{b}_{+}^{ \pm 1}, \widetilde{b}_{-}^{ \pm 1} \in \bigcap_{k=0}^{m-1} C^{\mu, k}\left(\Omega, S^{n-2}, C^{\nu-k}\left(\Gamma_{0}\right)\right)$ and $\widetilde{b}_{+}^{ \pm 1}\left(x, \omega^{\prime}, z\right), \widetilde{b}_{-}^{ \pm 1}\left(x, \omega^{\prime}, z^{-1}\right)$ have uniformly bounded analytic extensions for $|z|<1$. The partial indices are zero due to the positive definiteness of $\widetilde{b}\left(x, \omega^{\prime}, \zeta\right)$ (see $(2.2)$ and $(2.5))$ as is well known (see $[5,30])$. The $C^{\mu, k_{-}}$smooth dependence on the parameters $\left(x, \omega^{\prime}\right) \in\left(\bar{\Omega}, S^{n-2}\right)$ follows also from the fact that 
the partial indices are zero in the factorization (2.6) as is proved in [45].

Representation (2.3) follows from the positive homogeneity of $a_{ \pm}(x, \xi)$ with respect to $\xi$ and from (2.6) if we transform the variables

$$
\zeta=-\frac{\xi_{n}-i\left|\xi^{\prime}\right|}{\xi_{n}+i\left|\xi^{\prime}\right|}, \quad \omega^{\prime}=\left|\xi^{\prime}\right|^{-1} \xi^{\prime}, \quad t=\left|\xi^{\prime}\right|
$$

and factorize

$$
|\xi|^{r}=\left(\xi_{n}-i\left|\xi^{\prime}\right|\right)^{\frac{r}{2}}\left(\xi_{n}+i\left|\xi^{\prime}\right|\right)^{\frac{r}{2}}
$$

The estimates (2.4) follow from similar estimates for $\widetilde{b}_{ \pm}\left(x, \omega^{\prime}, \zeta\right)$ if the transformation (2.7) is taken into account. The homogeneity $b_{ \pm}(x, \lambda \xi)=b_{ \pm}(x, \xi)$ for $\lambda>0$ follows since the functions $\widetilde{b}_{ \pm}\left(x, \omega^{\prime}, \zeta\right)$ are independent of $t=\left|\xi^{\prime}\right|$.

Lacksquare

Remark 2.2. Theorem 2.1 remains valid if (2.2) is replaced by any other condition which ensures vanishing of the corresponding partial indices of the factorization (2.6). Some of such conditions can be found in [13] and in [30].

Lemma 2.3. Let $a(x, \xi)$ be as in Theorem 2.1 with $2 \leq m \leq \infty$ and let $b_{+}(x, \xi)$ be the factor in (2.3). Then the inverse has the expansion

$$
b_{+}^{-1}(x, \xi)=\sum_{k=0}^{l-1}\left(\frac{\left|\xi^{\prime}\right|}{\xi_{n}+i\left|\xi^{\prime}\right|}\right)^{k} b_{k}\left(x, \xi^{\prime}\right)+\left(\frac{\left|\xi^{\prime}\right|}{\xi_{n}+i\left|\xi^{\prime}\right|}\right)^{l} b_{l}^{0}(x, \xi)
$$

where $1 \leq l \leq m, \xi=\left(\xi^{\prime}, \xi_{n}\right) \in \mathbb{R}^{n}$ and where $b_{k} \in H^{0} C^{\mu, m-k-1}\left(\Omega, \mathbb{R}^{n-1}\right)$ for $k=0, \ldots, l-1$. The remainder $b_{l}^{0}(x, \xi)$ satisfies the estimates

$$
\begin{gathered}
\sup \left\{\left|\xi^{\beta} \partial_{x}^{\alpha} \partial_{\xi}^{\beta} b_{l}^{0}(x, \xi)\right|: x \in \Omega, \quad \xi \in \mathbb{R}^{n}, \quad|\alpha| \leq \mu\right. \\
|\beta| \leq m-l-1\}<\infty
\end{gathered}
$$

Moreover, $b_{l}^{0}\left(x, \xi^{\prime}, \xi_{n}+i \lambda\right)$ has an uniformly bounded analytic extension for $\lambda>0$.

Proof. The function $\widetilde{b}_{+}^{-1}\left(x, t \omega^{\prime}, \zeta\right)$ with the transformed variables belongs to the space $C^{\mu, m-1}\left(\Omega, \mathbb{R}^{+} \times S^{n-2} \times \Gamma_{0}\right)$ and has an analytic extension into the disc $|\zeta|<1$ (see (2.6)). Then $\widetilde{b}_{+}^{-1}\left(x, t \omega^{\prime}, 1+\theta(\zeta-1)\right.$ ) is correctly defined for $0 \leq \theta \leq 1$. If we apply Taylor's formula about 
$\theta=0$ and then take $\theta=1$, we get

$$
\begin{array}{r}
\widetilde{b}_{+}^{-1}\left(x, t \omega^{\prime}, \zeta\right)=\sum_{k=0}^{l-1} \frac{\widetilde{b}_{k}^{1}\left(x, t \omega^{\prime}, 1\right)}{k !}(\zeta-1)^{k}+(\zeta-1)^{r} \widetilde{b}_{l}^{0}\left(x, t \omega^{\prime}, \zeta\right) \\
\text { with } \widetilde{b}_{k}^{1}\left(x, t \omega^{\prime}, \zeta\right)=\partial_{\zeta}^{k} \widetilde{b}_{+}^{-1}\left(x, t \omega^{\prime}, \zeta\right)
\end{array}
$$

and the remainder $\widetilde{b}_{l}^{0}\left(x, t \omega^{\prime}, \zeta\right)=\frac{1}{l !} \int_{0}^{1}(1-\tau)^{l} \widetilde{b}_{l}^{1}\left(x, t \omega^{\prime}, 1+\tau(\zeta-1)\right) d \tau$.

If we insert $\zeta$ given in (2.7), the latter representation yields (2.8) and (2.9).

2.2. MODIFICATION OF SYMBOLS. If $a \in H^{r} C^{\mu, m}\left(\mathbb{R}^{n}, \mathbb{R}^{n}\right)$, $r<0$, then the operator $a(x, \partial): \mathbb{H}_{p}^{s}\left(\mathbb{R}^{n}\right) \rightarrow \mathbb{H}_{p}^{s-r}\left(\mathbb{R}^{n}\right)$ is unbounded even if $\mu=m=\infty$ (e.g., $|\partial|^{r}:=W_{|\xi|^{r}}^{0}$ is unbounded even in $L_{p}\left(\mathbb{R}^{n}\right)$; see [46, Section VI]). Therefore, we suggest the following modification of symbols:

$$
\stackrel{\circ}{a}(x, \xi)=a\left(x,\left(1+\left|\xi^{\prime}\right|^{2}\right)^{1 / 2}\left|\xi^{\prime}\right|^{-1} \xi^{\prime}, \xi_{n}\right) .
$$

The modification (2.10) differs slightly from the one introduced by G.Eskin in [14, p.91] and suits better our purposes ${ }^{7}$.

Then, due to Corollary 1.6, for $a \in H^{r} C^{\mu, m}\left(\mathbb{R}^{n}, \mathbb{R}^{n}\right)$, where $\mu \geq l+n$, $m>n / 2, \quad l=\max \{|s|,|s-r|\}, s, r \in \mathbb{R}$, the operator

$$
r_{\Omega} \stackrel{\circ}{a}(x, \partial): \mathbb{H}_{p}^{s}\left(\mathbb{R}^{n}\right) \rightarrow \mathbb{H}_{p}^{s-r}\left(\mathbb{R}^{n}\right)
$$

is bounded for any compact $\Omega \Subset \mathbb{R}^{n}$ and any $r<0$.

Corollary 2.4. If $a(x, \xi)$ satisfies the conditions of Theorem 2.1, then the modified factors $\stackrel{\circ}{a}_{ \pm}(x, \xi)(\operatorname{see}(2.3))$ belong to the class $\mathbb{S}_{r}^{\mu, m-1}\left(\Omega, \mathbb{R}^{n-1} \times \mathbb{R}\right)$ defined in $(1.26)$.

Note that the modification (2.10) does not change the principal symbol of a pseudo-differential operator. In fact, we have the following lemma.

Lemma 2.5. Let $\Omega \Subset \mathbb{R}^{n}$ be any compact domain, $a \in H^{r} C^{\mu, m}\left(\Omega, \mathbb{R}^{n}\right)$ and

${ }^{7}$ The modification $\stackrel{\circ}{a}(x, \xi)=a\left(x,\left(1+\left|\xi^{\prime}\right|\right)\left|\xi^{\prime}\right|^{-1} \xi^{\prime}, \xi_{n}\right)$ introduced in [14, p.91] has discontinuous derivatives $\partial_{\xi^{\prime}}^{\alpha^{\prime}} \stackrel{\circ}{a}(x, \xi)$ for $\left|\alpha^{\prime}\right|>1$ as a function of $\xi^{\prime}$. 
$\mu \geq l+n, \quad m>n / 2+1, \quad l=\max \{|s|,|s-r+1|\}, \quad s \in \mathbb{R}$, $-n+1<r<\infty$. Let the symbol satisfy

$$
\left|\partial_{x}^{\alpha} \partial_{\xi}^{\beta} a(x, \xi)\right| \leq M_{\alpha, \beta}|\xi|^{r-\beta_{n}-\delta}\left|\xi^{\prime}\right|^{\delta-\left|\beta^{\prime}\right|} \text { for } x \in \Omega \text { and } \xi \in \mathbb{R}^{n}
$$

and for any choice of multi-indices $|\alpha| \leq \mu$ and $|\beta| \leq m-1$. Here $\delta=0$ if $\beta^{\prime}=0$ and $\delta=1$ if $\beta^{\prime} \neq 0$.

If $1<p<\infty$ then the operator

$$
A_{\Omega}=r_{\Omega} a(x, \partial)-r_{\Omega} \stackrel{\circ}{a}(x, \partial): \widetilde{\mathbb{H}}_{p}^{s}(\Omega) \rightarrow \mathbb{H}_{p}^{s-r+1}(\Omega)
$$

is bounded, i.e. $A_{\Omega}$ has the order $r-1$.

Proof. Since, with the mean value theorem,

$a(x, \xi)-\stackrel{\circ}{a}(x, \xi)=a\left(x, \xi^{\prime}, \xi_{n}\right)-a\left(x, \xi^{\prime}+\omega^{\prime}, \xi_{n}\right)=-\sum_{k=1}^{n-1} \frac{\partial a\left(x, \xi^{\prime}+\theta \omega^{\prime}, \xi_{n}\right)}{\partial \xi_{k}} \omega_{k}$,

with a suitable $0<\theta<1, \quad \omega^{\prime}=\left[\left(1+\left|\xi^{\prime}\right|^{2}\right)^{1 / 2}-\left|\xi^{\prime}\right|\right]\left|\xi^{\prime}\right|^{-1} \xi^{\prime}$

and

$\frac{1}{2}\left(\left|\xi^{\prime}\right|+\left|\xi_{n}\right|\right) \leq\left|\xi^{\prime}+\theta \omega^{\prime}\right|+\left|\xi_{n}\right| \leq 2\left(\left|\xi^{\prime}\right|+\left|\xi_{n}\right|\right) \quad$ for $\quad\left|\omega^{\prime}\right| \leq 1$ provided $\left|\xi^{\prime}\right|+\left|\xi_{n}\right| \geq 2$

we get with (2.12)

$\left|\partial_{x}^{\alpha} \partial_{\xi}^{\beta}[a(x, \xi)-\stackrel{\circ}{a}(x, \xi)]\right| \leq M_{\alpha, \beta}^{\prime}|\xi|^{r-\beta_{n}-1}\left|\xi^{\prime}\right|^{1-\left|\beta^{\prime}\right|}$ for all $x \in \Omega$ and $\xi \in \mathbb{R}^{n}$.

Therefore, the operator

$$
r_{\Omega} a_{1}(x, \partial): \widetilde{\mathbb{H}}_{p}^{s}(\Omega) \rightarrow \mathbb{H}_{p}^{s-r+1}(\Omega)
$$

is bounded with

$$
a_{1}(x, \xi)=\left[1-\chi_{0}(\xi)\right][a(x, \xi)-\stackrel{\circ}{a}(x, \xi)]|\xi|^{-r+1}
$$

where $\chi_{0} \in C_{0}^{\infty}\left(\mathbb{R}^{n}\right)$ is a cut-off function with $\chi_{0}(\xi)=1$ if $|\xi|<1$. Since the function

$$
a_{2}(x, \xi):=\chi_{0}(\xi)[a(x, \xi)-\stackrel{\circ}{a}(x, \xi)]
$$

is compactly supported in $\xi$, we get

$$
r_{\Omega} a_{2}(x, \partial) u \in C^{\infty}(\Omega) \subset \mathbb{B}_{p}^{s-r+1}(\Omega) \quad \text { for any } \quad u \in \widetilde{\mathbb{H}}_{p}^{s}(\Omega) .
$$

Further, we use the representation

$$
A_{\Omega}=r_{\Omega} a_{1}(x, \partial) \chi_{0}|\partial|^{r-1}+r_{\Omega} a_{1}(x, \partial)\left(1-\chi_{0}\right)|\partial|^{r-1}+r_{\Omega} a_{2}(x, \partial),
$$


where $|\partial|^{r}=W_{|\xi|^{r}}^{0}$ and $|\xi|^{r-1} \in H^{r-1} C^{\infty}\left(\mathbb{R}^{n}\right)$ for $r-1 \geq 0$. For $-n<r-1<0$ these operators are the Riesz potentials and can be written as

$$
|\partial|^{r-1} \varphi(x)=c_{r-1} \int_{\mathbb{R}^{n}} \frac{\varphi(y) d y}{|y-x|^{n+r-1}} \quad \text { with } \quad c_{r-1}=\frac{2^{r-1} \Gamma\left(\frac{n+r-1}{2}\right)}{\pi^{\frac{n}{2}} \Gamma\left(\frac{n}{2}\right)(2 .}
$$

(see [46, Chapter V]). Due to Corollary 1.6 it suffices to consider only the second term in (2.15) and assume $-n<r-1<0$. Then the operator $|\partial|^{r-1}: \mathbb{H}_{p}^{s}\left(\mathbb{R}^{n}\right) \rightarrow \mathbb{H}_{p}^{s-r+1}\left(\mathbb{R}^{n}\right)$ is bounded for $r-1 \geq 0$ and $\chi_{0}|\partial|^{r-1}: \mathbb{H}_{p}^{s}\left(\mathbb{R}^{n}\right) \rightarrow \mathbb{H}_{p}^{s-r+1}\left(\mathbb{R}^{n}\right)$ is bounded for $-n<r-1<0 \quad(s \in \mathbb{R}$ and $1<p<\infty)$.

Without loss of generality we may suppose $r_{\Omega} \chi_{0}=1$. Then due to (2.16), $\psi=\left(1-\chi_{0}\right)|\partial|^{r-1} \varphi \in C^{\infty}\left(\mathbb{R}^{n}\right)$ and

$$
\left|\partial_{x}^{\alpha} \psi(x)\right| \leq M_{\alpha, \varphi}\left(1+|x|^{2}\right)^{-\frac{n+r-1+|\alpha|}{2}} \text { for } \varphi \in \widetilde{\mathbb{H}}_{p}^{s}(\Omega) .
$$

This is evident for $s \geq 0$ since $\widetilde{\mathbb{H}}_{p}^{s}(\Omega) \subset L_{p}(\Omega)$, whereas for $s<0$ we can apply the representation $\varphi=\sum_{|\beta|<|s|+1} c_{\beta} \partial_{x}^{\beta} \varphi_{0} \quad$ with $\quad \varphi_{0} \in$ $L_{p}(\Omega)$ (recall that $|\partial|^{r-1} \partial_{x}^{\beta}=\partial_{x}^{\beta}|\partial|^{r-1}$ ). The above estimates imply $\psi \in \bigcap_{q>\frac{n}{n+r-1}} \mathbb{H}_{q}^{s}\left(\mathbb{R}^{n}\right)$ and, therefore, $r_{\Omega} a_{1}(x, \partial)\left(1-\chi_{0}\right)|\partial|^{r-1} \varphi \in$ $\bigcap_{q>\frac{n}{n+r-1}} \mathbb{H}_{q}^{\nu}(\Omega) \subset \bigcap_{1<q<\infty} \mathbb{H}_{q}^{\nu}(\Omega)$ if $|\nu| \leq l . \quad$ Lacksquare

Remark 2.6. If $l=\max \{|s|,|s-r|\}$ and all the conditions of Lemma 2.5 are fulfilled, then the operator

$$
r_{\Omega} a(x, \partial): \widetilde{\mathbb{H}}_{p}^{s}(\Omega) \rightarrow \mathbb{H}_{p}^{s-r}(\Omega)
$$

is bounded.

2.3. SOLVABILITY RESULTS. Let us consider an $N \times N$ system of pseudo-differential equations on a compact domain $\Omega \Subset \mathbb{R}^{n}$ with smooth boundary $\partial \Omega$ :

$$
r_{\Omega} a(x, \partial) u=v
$$

with a symbol $a \in H_{T}^{r} C^{k, \mu}\left(\Omega, \mathbb{R}^{n}\right)$, where $\mu>\frac{n}{2}+2,-n<r<\infty$, $-\infty<s<k, 1<p<\infty$ and seek a solution $u \in \widetilde{\mathbb{H}}_{p}^{s}(\Omega)$ for any given $v \in \mathbb{H}_{p}^{s-r}(\Omega)$.

The next theorem has been proved by R.Duduchava, D.Natroshvili and E. Shargorodsky in $[11,12]$ for the particular case of pseudodifferential equations arising in elasticity (see Theorems 3.3-3.4 below) and in [13, Theorem 3.26] for more general symbols but less general spaces where $p=2$. 
Theorem 2.7. Let the symbol $a(x, \xi)$ in $(2.18)$ be elliptic, i.e.

$$
\inf \left\{|\operatorname{det} a(x, \xi)|: x \in \Omega, \quad \xi \in S^{n-1}\right\}>0,
$$

and positive definite on the boundary $\partial \Omega$, satisfying condition (2.2) for all $x \in \partial \Omega$.

Then equation (2.18) is Fredholm if and only if the conditions

$$
\frac{1}{p}+\frac{r}{2}-1<s<\frac{1}{p}+\frac{r}{2}
$$

are fulfilled.

If the numerical range of the matrix symbol $a(x, \xi)$, i.e.

$$
\mathcal{R}_{x}(a):=\left\{(a(x, \omega) \theta, \theta): \omega \in \mathbb{R}^{n}, \quad \theta \in \mathbb{C}^{N},|\omega|=|\theta|=1\right\},
$$

does not contain any half-line $\left\{z \in \mathbb{C}: \arg z=\alpha_{x} \in[0,2 \pi)\right\}$ for all interior points $x \in \Omega$, then the index of equation (2.18) is zero.

If, in addition, the homogeneous equation $r_{\Omega} a(x, \partial) u=0$ has only the trivial solution $u=0$ in one of the spaces $\widetilde{\mathbb{H}}_{p}^{s}(\Omega)$ where $s$ and $p$ satisfy conditions (2.20), then (2.18) has a unique solution in all these spaces.

Proof. For the boundedness of the operator in (2.18) see Lemma 2.5 and Remark 2.6.

If the local principle is applied (see, e.g. [9, 13]), we find out that $r_{\Omega} a(x, \partial)$ in $(2.18)$ is a Fredholm operator if and only if its local representatives

$$
\begin{array}{rll}
\stackrel{\circ}{a}\left(x_{0}, \partial\right) & : & \mathbb{H}_{p}^{s}\left(\mathbb{R}^{n}\right) \rightarrow \mathbb{H}_{p}^{s-r}\left(\mathbb{R}^{n}\right) \quad \text { if } x_{0} \in \Omega, \\
r_{+} \stackrel{\circ}{a}\left(x_{0}, \partial\right) & : & \widetilde{\mathbb{H}}_{p}^{s}\left(\mathbb{R}_{+}^{n}\right) \rightarrow \mathbb{H}_{p}^{s-r}\left(\mathbb{R}_{+}^{n}\right) \quad \text { if } x_{0} \in \partial \Omega, \\
\stackrel{\circ}{a}\left(x_{0}, \xi\right) & :=a\left(x_{0},\left(1+\left|\xi^{\prime}\right|^{2}\right)^{\frac{1}{2}}\left|\xi^{\prime}\right|^{-1} \xi^{\prime}, \xi_{n}\right)
\end{array}
$$

are invertible for every $x_{0} \in \bar{\Omega}=\Omega \cup \partial \Omega$ (see [13]). If $x_{0} \in \Omega$, then the invertibility of $\stackrel{\circ}{a}\left(x_{0}, \partial\right)$ in $(2.21)$ follows from the ellipticity condition (2.19). The inverse is then given by $\stackrel{\circ}{a}^{-1}\left(x_{0}, \partial\right)$.

If $x_{0} \in \partial \Omega$, then the ellipticity condition (2.19) is only necessary but not sufficient for $r_{+} \stackrel{\circ}{a}\left(x_{0}, \partial\right)$ in $(2.22)$ to be invertible. The lifted operator

$$
\begin{aligned}
& r_{+} \lambda_{-}^{s-r}(\partial) \ell r_{+} \stackrel{\circ}{a}\left(x_{0} \partial\right) \lambda_{+}^{-s}(\partial) \\
& \quad=r_{+}\left(\lambda_{-}^{s-r} \stackrel{\circ}{a} \lambda_{+}^{-s}\right)\left(x_{0}, \partial\right): L_{p}\left(\mathbb{R}_{+}^{2}\right) \rightarrow L_{p}\left(\mathbb{R}_{+}^{2}\right)
\end{aligned}
$$

should be invertible as well (cf.(1.4), (1.24), (1.25) and [13]). Since

$$
\lambda_{-}^{\nu+1}(\xi) \stackrel{\circ}{a}\left(x_{0}, \xi\right) \lambda_{+}^{-\nu}(\xi)=\left(\frac{\xi_{n}-i\left(1+\left|\xi^{\prime}\right|^{2}\right)^{\frac{1}{2}}}{\xi_{n}+i\left(1+\left|\xi^{\prime}\right|^{2}\right)^{\frac{1}{2}}}\right)^{s-\frac{r}{2}} \stackrel{\circ}{a}_{-}\left(x_{0}, \xi\right) \stackrel{\circ}{a_{+}}\left(x_{0}, \xi\right)
$$


(cf. Theorem 2.1) where $a_{ \pm}, a_{ \pm}^{-1} \in H^{0} C^{k-1}\left(\mathbb{R}^{2}\right)$ have all the necessary analyticity properties, the operator in (2.23) is invertible if and only if there hold the conditions

$$
\frac{1}{p}-1<s-\frac{r}{2}<\frac{1}{p}
$$

(cf. [9, Sect.2]). Note that conditions (2.25) and (2.20) coincide.

The operator $r_{\Omega} a(x, \partial)$ has one and the same regularizer in all the spaces $\mathbb{H}_{p}^{s}(\Omega) \rightarrow \widetilde{\mathbb{H}}_{p}^{s-r}(\Omega)$ if $(2.25)$ holds. This implies the independence of $\operatorname{Ker} r_{\Omega} a(x, \partial)$ of $r$ and $p$. Hence, if the kernel is trivial for some pair of parameters $(s, p)$, it will be trivial for any $s$ and $p$ meeting conditions (2.25).

The index formula Ind $r_{\Omega} a(x, \partial)=0$ follows, since the index as well as the kernel are independent of $s$ and $p$ and for $s=-1 / 2, p=2$ this is known from [13, Theorem 3.26]. If, in addition, we assume the property $\operatorname{Ker} r_{\Omega} a(x, \partial)=\{0\}$, this yields the unique solvability of Equation(2.18).

Lacksquare

Remark 2.8. Theorem 2.7 holds if we consider the same equation in the Besov spaces: Let $a \in H_{T}^{r} C^{k, \mu}\left(\Omega, \mathbb{R}^{n}\right)$ and $v \in \mathbb{B}_{p, q}^{s-r}(\Omega)$ be given and seek a solution $u \in \widetilde{\mathbb{B}}_{p, q}^{s}(\Omega) \quad(\operatorname{see}(2.18))$. Then the condition (2.20) for the Fredholm property is independent of $q$.

The proof of sufficiency follows from Theorem 2.7 and from the interpolation property $(1.11)-(1.12)$ since the operator $r_{\Omega} a(x, \partial)$ : $\widetilde{\mathbb{B}}_{p, q}^{s}(\Omega) \rightarrow \mathbb{B}_{p, q}^{s-r}(\Omega)$ and its inverse $\left(r_{\Omega} a\right)^{-1}(x, \partial): \mathbb{B}_{p, q}^{s-r}(\Omega) \rightarrow \widetilde{\mathbb{B}}_{p, q}^{s}(\Omega)$ are bounded for the same values of the parameters $s$ and $p$ as in Theorem 2.7 .

The necessity can be seen as follows: for $s$ outside of the interval given by inequality (2.21) either the equation (for some small $s$ ) or the adjoint one (for some big $s$ ) has an infinite-dimensional kernel. If $s$ coincides with one of the bounds in (2.20) then the stability of the Fredholm property yields that $r_{\Omega}(x, \partial)$ can not be normally solvable. 


\section{CRACK PROBLEMS: SOLVABILITY AND REGULARITY}

3.1. FORMULATION OF PROBLEMS. Now we shall apply the refined Wiener-Hopf technique to the problem of finding the displacement field $u=\left(u_{1}, u_{2}, u_{3}\right)$ in a homogeneous anisotropic elastic medium occupying a domain $\Omega \subset \mathbb{R}^{3}$ and containing a crack $S \subset \Omega$ whose geometry is given. Let us assume that the crack lies in the interior, $\partial \Omega \cap \bar{S}=\varnothing$, where $\bar{S}=S \cup \partial S$. Assume that the closure $\bar{S}$ can be represented as a $k$-smooth manifold $(k \geq 3)$ with a $k$-smooth boundary $\partial S$. For the sake of brevity we suppose that $\Omega=\mathbb{R}^{3}$. Moreover, $S$ can be extended to a closed compact $k$-smooth surface $\widetilde{S} \subset \mathbb{R}^{3}$ with $S \subset \widetilde{S}$ and $\widetilde{S}=\partial \widetilde{\Omega}^{+}$where $\widetilde{\Omega}^{+}$is a domain interior to $\widetilde{S}$. The equilibrium equations in the elastic material read (see [15, 27, 29])

$$
\sum_{j} \frac{\partial \tau_{i j}}{\partial x_{i j}}+X_{i}=0, \quad i=1,2,3
$$

where $X=\left(X_{1}, X_{2}, X_{3}\right)$ are given body forces and where $\tau=\left\|\tau_{i j}\right\|_{3 \times 3}$ is the stress tensor. In the sequel we assume that $X=0$ since otherwise we can superpose a corresponding particular elastic field.

For small deformations we introduce the strain tensor $e=\left\|e_{i j}\right\|_{3 \times 3}$ and assume Hooke's law in the form (see [15, 27, 29])

$$
\begin{aligned}
\tau_{i j} & =\sum_{m, n=1}^{3} c_{i j m n} e_{m n} \\
\text { where } \quad e_{m n} & =\frac{1}{2}\left(\frac{\partial u_{m}}{\partial x_{n}}+\frac{\partial u_{n}}{\partial x_{m}}\right) \quad \text { for } \quad i, j, m, n=1,2,3 .
\end{aligned}
$$

Here the elastic moduli $c_{i j m n}$ are real-valued and satisfy the symmetry relations

$$
c_{i j m n}=c_{m n i j}=c_{j i m n} .
$$

The energy conservation law leads to the following strong ellipticity condition: there exists a constant $c_{0}>0$ such that

$$
\sum_{i, j, m, n} c_{i j m n} \zeta_{i j} \bar{\zeta}_{m n} \geq c_{0} \sum_{i, j}\left|\zeta_{i j}\right|^{2} \quad \text { for all } \quad \zeta_{i j}=\zeta_{j i} \in \mathbb{C}
$$


From (3.3) it follows that the $6 \times 6$ matrix

$$
\mathcal{C}=\left\|c_{i j}\right\|_{6 \times 6}:=\left(\begin{array}{cccccc}
c_{1111} & c_{1122} & c_{1133} & c_{1123} & c_{1131} & c_{1112} \\
\bullet & c_{222} & c_{2233} & c_{2223} & c_{2231} & c_{2212} \\
\bullet & \bullet & c_{3333} & c_{3323} & c_{3331} & c_{3312} \\
\bullet & \bullet & \bullet & c_{2323} & c_{2331} & c_{2312} \\
\bullet & \bullet & \bullet & \bullet & c_{3131} & c_{3112} \\
\bullet & \bullet & \bullet & \bullet & \bullet & c_{1212}
\end{array}\right) \text {, }
$$

defined by the 21 elastic moduli (see (3.1), (3.2)) is symmetric

$$
\mathcal{C}=\left\|c_{i j}\right\|_{6 \times 6}=\left\|c_{j i}\right\|_{6 \times 6}=\mathcal{C}^{\top}
$$

and positive definite: there exists a positive $c_{0}$ such that

$$
\zeta^{\top} \mathcal{C} \bar{\zeta}=\sum_{i, j} c_{i j} \zeta_{i} \overline{\zeta_{j}} \geq \frac{c_{0}}{2} \sum_{j}\left|\zeta_{j}\right|^{2}=\frac{c_{0}}{2}|\zeta|^{2} \quad \text { for all } \zeta \in \mathbb{C}^{6}
$$

After inserting the representation of the strain tensor into Hooke's law (3.1) and the latter into the equilibrium equations, we get for the displacement vector $u$ the second order partial differential equation (see [15] and [29, Section 11])

$$
\mathcal{L} u=0 \quad \text { with } \quad \mathcal{L}=\mathbf{D}^{\top} \mathcal{C} \mathbf{D} .
$$

Here $\mathbf{D}$ represents the generalized $6 \times 3$ matrix operator whose transposed $3 \times 6$ matrix operator $\mathbf{D}^{\top}$ is given by

$$
\mathbf{D}^{\top}=\left(\begin{array}{cccccc}
\partial_{1} & 0 & 0 & 0 & \partial_{3} & \partial_{2} \\
0 & \partial_{2} & 0 & \partial_{3} & 0 & \partial_{1} \\
0 & 0 & \partial_{3} & \partial_{2} & \partial_{1} & 0
\end{array}\right), \quad \text { where } \quad \partial_{j}=\frac{\partial}{\partial x_{j}}
$$

For the elliptic partial differential equation (3.6) we shall consider two fundamental boundary value problems: the Dirichlet and the Neumann problems.

The Dirichlet Problem. For given $u^{ \pm} \in \mathbb{H}_{\not /}^{\nVdash / \not}(\mathbb{S})$ with $\left(u^{+}-u^{-}\right) \in$ $\widetilde{\mathbb{H}}_{2}^{1 / 2}(S)$ find a solution of equation (3.6) in $\mathbb{R}^{3} \backslash \bar{S}$ satisfying on $S$ the boundary conditions

$$
\gamma_{S}^{ \pm} u=u^{ \pm}
$$

Here $\gamma_{S}^{ \pm}$are the trace operators which restrict functions from the Sobolev space $\mathbb{H}_{\text {loc }}^{1}\left(\mathbb{R}_{S}^{3}\right)$ with $\mathbb{R}_{S}^{3}:=\mathbb{R}^{3} \backslash S$ to the two different faces $S^{ \pm}$of the surface $S$ where $S^{+}$represents the face associated with the compact interior domain $\widetilde{\Omega}^{+}$. By $\mathbb{H}_{l o c}^{1}\left(\mathbb{R}_{S}^{3}\right)$ we denote the Sobolev space of vector-functions $\varphi(x)=\left(\varphi_{1}(x), \varphi_{2}(x), \varphi_{3}(x)\right)$ on $\mathbb{R}_{S}^{3}$ with

$$
(\varphi, \varphi)_{\Omega_{S}}^{(1)}=\left(\int_{\Omega_{S}}\left(|\varphi(x)|^{2}+|\nabla \varphi(x)|^{2}\right) d x\right)^{1 / 2}<\infty
$$


for any compact region $\Omega_{S}=\Omega \backslash S \Subset \mathbb{R}^{3}$. At infinity we require the solutions $u(x)$ vanish:

$$
u(x)=o(1) \quad \text { for } \quad|x| \rightarrow \infty .
$$

$\mathbb{H}_{2}^{1 / 2}(S)$ denotes the trace space of $\mathbb{H}_{l o c}^{1}\left(\mathbb{R}_{S}^{3}\right)$-functions on $S$; note that the traces $\gamma_{S}^{+} \varphi$ and $\gamma_{S}^{-} \varphi$ for $\left.\varphi \in \mathbb{H}_{l o c}^{1}\left(\mathbb{R}_{S}^{3}\right)\right)$ may be different. As introduced previously, $\widetilde{\mathbb{H}}_{2}^{1 / 2}(S)$ consists of those functions $\psi \in \mathbb{H}_{2}^{1 / 2}(\widetilde{S})$ for which $\operatorname{supp} \psi \subset \bar{S}$.

The Neumann Problem. For given $t^{ \pm} \in \mathbb{H}_{\not \nvdash}^{-\nVdash / \not}(\mathbb{S})$ with $\left(t^{+}-\right.$ $\left.t^{-}\right) \in \widetilde{\mathbb{H}}_{2}^{-1 / 2}(S)$ find a solution of equation (3.6) in $\mathbb{R}^{3} \backslash \bar{S}$ satisfying the boundary conditions

$$
\gamma_{S}^{ \pm} \mathcal{T}\left(\partial_{x}, n(x)\right) u=t^{ \pm} \text {on } S .
$$

Here $\mathcal{T}\left(\partial_{x}, n(x)\right)$ denotes the traction operator (see [29, Sect.11]) defined by

$$
\begin{gathered}
\mathcal{T}\left(\partial_{x}, n(x)\right) u=\mathcal{N}^{\top} \mathcal{C} \mathbf{D} u \\
\text { where } \quad \mathcal{N}^{\top}=\left(\begin{array}{cccccc}
n_{1} & 0 & 0 & 0 & n_{3} & n_{2} \\
0 & n_{2} & 0 & n_{3} & 0 & n_{1} \\
0 & 0 & n_{3} & n_{2} & n_{1} & 0
\end{array}\right)
\end{gathered}
$$

with $n_{j}=n_{j}(x)$ and $n(x)=\left(n_{1}(x), n_{2}(x), n_{3}(x)\right)$ representing the exterior normal vector to $\widetilde{S}$.

Since the pointwise traces $\gamma_{S}^{ \pm} \mathcal{T}\left(\partial_{x}, n(x)\right) u$ in (3.10) may not exist for $u \in \mathbb{H}_{l o c}^{1}\left(\mathbb{R}_{S}^{3}\right)$, we need to introduce this expression via the variational formulation of the problem.

For any regular vectors $u, v \in C^{2}\left(\mathbb{R}_{\widetilde{S}}^{3}\right) \cap C^{1}\left(\overline{\widetilde{\Omega}^{-}}\right) \bigcap C^{1}\left(\overline{\widetilde{\Omega}^{+}}\right)$with $\widetilde{\Omega}^{-}:=$ $\mathbb{R}^{3} \backslash \overline{\Omega^{+}}$, decaying at infinity as $|x|^{-1}$, there holds the Green formula

$$
\begin{aligned}
\int_{\widetilde{S}} \gamma_{\widetilde{S}}^{ \pm} \mathcal{T}\left(\partial_{x}, n(x)\right) u(x) \gamma_{\widetilde{S}}^{ \pm} v(x) & = \pm \int_{\widetilde{\Omega}^{ \pm}} \mathcal{L} u(x) v(x) d x \pm \int_{\widetilde{\Omega}^{ \pm}} E(u, v) d x \\
\text { with } \quad E(u, v) & :=\sum_{k, j, p, q} c_{k j p q} \partial_{j} u_{k} \partial_{q} v_{p}
\end{aligned}
$$

(see [29]). If $u \in \mathbb{H}_{l o c}^{1}\left(\mathbb{R}_{\widetilde{S}}^{3}\right), \quad \mathcal{L} u \in L_{2, l o c}\left(\mathbb{R}_{\widetilde{S}}^{3}\right)$ then the right hand side in (3.12) is correctly defined for any $v \in \mathbb{H}_{\text {comp }}^{1}\left(\mathbb{R}_{\widetilde{S}}^{3}\right)$. The bilinear form $\left.\left(\gamma_{\widetilde{S}}^{ \pm} \mathcal{T}(\partial, n)\right) u, \gamma_{\widetilde{S}}^{ \pm} v\right)_{\widetilde{S}}$ defines then by duality the distribution $\gamma_{\widetilde{S}}^{ \pm} \mathcal{T}(\partial, n) u \in \mathbb{H}_{2}^{-1 / 2}(\widetilde{S})$ (see [49]). Hence, (3.10) is understood in the distributional sense. 
To formulate the boundary integral equations we need the double and the single layer potentials

$$
\begin{aligned}
& \mathbf{U}_{S} \psi(z)=\int_{S}\left[\mathcal{T}\left(\partial_{y}, n(y)\right) \mathcal{G}(z-y)\right]^{\top} \psi(y) d_{y} S \\
& \mathbf{V}_{S} \psi(z)=-\int_{S} \mathcal{G}(z-y) \psi(y) d_{y} S \text { for } z \in \mathbb{R}_{S}^{3},
\end{aligned}
$$

respectively, where

$$
\mathcal{G}(x)=\mathcal{F}^{-1} \mathcal{L}^{-1}(x) \text { for } \quad x \in \mathbb{R}^{3}
$$

represents the fundamental solution of equation (3.6) given by the inverse Fourier transform of the inverse symbol $\mathcal{L}^{-1}(\xi)$ of the elliptic differential operator $\mathcal{L}$ in $(3.6)$.

If $\mathcal{D}(\xi)$ denotes the symbol of the operator $\mathbf{D}$, given by

$$
\mathcal{D}^{\top}(\xi)=-i\left(\begin{array}{cccccc}
\xi_{1} & 0 & 0 & 0 & \xi_{3} & \xi_{2} \\
0 & \xi_{2} & 0 & \xi_{3} & 0 & \xi_{1} \\
0 & 0 & \xi_{3} & \xi_{2} & \xi_{1} & 0
\end{array}\right) \quad \text { for } \quad \xi=\left(\xi_{1}, \xi_{2}, \xi_{3}\right) \in \mathbb{R}^{3}
$$

then the symbol of $\mathcal{L}$ is given by

$$
\mathcal{L}(\xi)=\mathcal{D}^{\top}(\xi) \mathcal{C D}(\xi)
$$

Obviously, $\mathcal{D}(\xi)$ is homogeneous, i.e. $\mathcal{D}(\lambda \xi)=\lambda \mathcal{D}(\xi)$ and one easily shows that $\operatorname{rank} \mathcal{D}(\xi)=3$ for $|\xi| \neq 0$. Hence, there exists $c_{1}>0$ such that

$$
|\mathcal{D}(\xi) \zeta| \geq c_{1}|\xi \| \eta|
$$

and Condition (3.5) ensures the negative definiteness of the $3 \times 3$ matrix symbol $\mathcal{L}(\xi)$ in $(3.16)$ :

$$
-(\mathcal{L}(\xi) \zeta, \zeta)=-(\mathcal{C D}(\xi) \zeta, \mathcal{D}(\xi) \zeta) \geq \frac{c_{0}}{2}|\mathcal{D}(\xi) \zeta|^{2} \geq d_{0}|\xi|^{2}|\zeta|^{2}
$$

with some constant $d_{0}>0$ and for all $\xi \in \mathbb{R}^{3}, \quad \zeta \in \mathbb{C}^{3}$.

The same notations $\mathbf{U}_{S}$ and $\mathbf{V}_{S}$ will be used for the direct values of the integral operators (3.13) when $z \in S$; in this case the integral defining $\mathbf{U}_{S}$ is understood in the sense of a Cauchy v.p.. $\mathbf{U}_{S}$ and $\mathbf{V}_{S}$ represent pseudo-differential operators of orders 0 and -1 , respectively.

To reduce the boundary value problems to the solution of some boundary integral equations, we need the following two pseudo-differential 
operators

$$
\begin{gathered}
\mathbf{U}_{S}^{0} \psi(x)=\int_{S} \mathcal{T}\left(\partial_{x}, n(x)\right) \mathcal{G}(x-y) \psi(y) d_{y} S \\
\mathbf{D}_{S} \psi(x)=\mathcal{T}\left(\partial_{x}, n(x)\right) \int_{S}\left[\mathcal{T}\left(\partial_{y}, n(y)\right) \mathcal{G}(x-y)\right]^{\top} \psi(y) d_{y} S,(3.18)
\end{gathered}
$$

which have the orders 0 and +1 , respectively. Again, the integrals are understood in the sense of a Cauchy v.p..

The next two theorems are well-known (see $[7,11])$ and describe the explicit boundary integral equations equivalent to the Dirichlet and the Neumann crack problems (3.6), (3.7), (3.9) and (3.6), (3.9),(3.10), respectively.

Theorem 3.1. Let $u^{ \pm} \in \mathbb{H}_{\not \neq}^{\nVdash / \not k}(\mathbb{S})$ with $u_{0}:=\left(u^{+}-u^{-}\right) \in \widetilde{\mathbb{H}}_{2}^{1 / 2}(S)$ ( see (3.7)). Then $u \in \mathbb{H}_{\text {loc }}^{1}\left(\mathbb{R}_{S}^{3}\right)$ is the solution of the Dirichlet crack problem (3.6), (3.7), (3.9) if and only if

$$
u(z)=-V_{S} \varphi(z)+U_{S} u_{0}(z) \quad \text { for } \quad z \in \mathbb{R}_{S}^{3},
$$

where $\varphi \in \widetilde{\mathbb{H}}_{2}^{-1 / 2}(S)$ solves the pseudo-differential equation on $S$

$$
\mathbf{V}_{S} \varphi=g
$$

with the right-hand side given by $g=-\frac{1}{2}\left(u^{+}+u^{-}\right)+\mathbf{U}_{S}^{0} u_{0}$.

Theorem 3.2. Let $t^{ \pm} \in \mathbb{H}_{\not \nvdash}^{-\nVdash / \nvdash}(\mathbb{S})$ with $t_{0}:=\left(t^{+}-t^{-}\right) \in \widetilde{\mathbb{H}}_{2}^{-1 / 2}(S)$ (see (3.10)). Then $u \in \mathbb{H}_{\text {loc }}^{2}\left(\mathbb{R}_{S}^{3}\right)$ is the variational solution of the Neumann crack problem (3.6), (3.9), (3.10) if and only if

$$
u(z)=-\mathbf{V}_{S} t_{0}(z)+\mathbf{U}_{S} \psi(z) \quad \text { for } \quad z \in \mathbb{R}_{S}^{3},
$$

where $\psi \in \widetilde{\mathbb{H}}_{2}^{1 / 2}(S)$ solves the pseudo-differential (hypersingular) equation on $S$

$$
\mathbf{D}_{S} \psi=f
$$

with the right hand side given by $f=\frac{1}{2}\left(t^{+}+t^{-}\right)+\mathbf{U}_{S}^{0} t_{0}$.

\subsection{THE DIRICHLET PROBLEM.}

Theorem 3.3 (see $[11,12])$. The single layer potential operator in $(3.13)$

$$
\mathbf{V}_{S}: \widetilde{\mathbb{H}}_{p}^{\nu}(S) \rightarrow \mathbb{H}_{1}^{\nu+\nVdash}(\mathbb{S})
$$

is bounded for any $1<p<\infty$ and $-k+1 \leq \nu \leq k+1$. 
If $k \geq 4$, then (3.23) is a Fredholm operator if and only if the conditions

$$
\frac{1}{p}-\frac{3}{2}<\nu<\frac{1}{p}-\frac{1}{2}
$$

hold, in which case (3.20) has a unique solution $\varphi \in \widetilde{\mathbb{H}}_{p}^{\nu}(S)$ for any given $g \in \mathbb{H}_{1}^{\nu+\nVdash}(\mathbb{S})$.

Similarly, in the Besov spaces with $1 \leq q \leq \infty$ the operator

$$
\mathbf{V}_{S}: \widetilde{\mathbb{B}}_{p, q}^{\nu}(S) \rightarrow \mathbb{B}_{1,11}^{\nu+\nVdash}(\mathbb{S})
$$

is bounded. Under conditions (3.24), Equation (3.20) has a unique solution $\varphi \in \widetilde{\mathbb{B}}_{p, q}^{\nu}(S)$ for any given $g \in \mathbb{B}_{1,11}^{\nu+\nVdash}(\mathbb{S})$.

Let $u^{ \pm} \in \bigcap_{1<p<\infty} \mathbb{H}_{1}^{\mathbb{K} / \not \mid}(\mathbb{S})$ with $\left(u^{+}-u^{-}\right) \in \bigcap_{1<p<\infty} \widetilde{\mathbb{H}}_{p}^{1 / 2}(S)$. Then the displacement vector $u(x)$ in (3.19), which solves the Dirichlet crack problem, is a real analytic function in $\mathbb{R}_{S}^{3}$ vanishing at infinity. The traces $\gamma_{S}^{ \pm} u=u^{ \pm}$on both faces of the crack surface $S$ belong to every Hölder space $C^{\alpha}(S)$ for $0<\alpha<\frac{1}{2}$ and coincide on $\partial S: u^{-}(x)=u^{+}(x)$ for $x \in \partial S$.

Proof. Let

$$
S=\bigcup_{j=1}^{N} Y_{j}, \quad \varkappa_{j}: X_{j} \rightarrow Y_{j}, \quad X_{j} \subset \mathbb{R}_{+}^{2}:=\mathbb{R} \times \mathbb{R}^{+}, \quad j=1, \ldots, N
$$

be some $k$-smooth atlas of the surface $S \subset \mathbb{R}^{3}$ and let

$$
\begin{gathered}
\tilde{\varkappa}_{j}: \tilde{X}_{j} \rightarrow \tilde{Y}_{j}, \quad \tilde{X}_{j}, \tilde{Y}_{j} \subset \mathbb{R}^{3}, \quad \tilde{Y}_{j} \cap S=Y_{j}, \\
\tilde{X}_{j}=(-\varepsilon, \varepsilon) \times X_{j},\left.\quad \tilde{\varkappa}_{j}\right|_{X_{j}}=\varkappa_{j}, \quad j=1, \ldots, N,
\end{gathered}
$$

be extensions of the diffeomorphisms in (3.26). By $d \varkappa_{j}(t)=\varkappa_{j}^{\prime}(t)$ and $d \widetilde{\varkappa}_{j}(\widetilde{t})=\widetilde{\varkappa}_{j}^{\prime}(\widetilde{t})$ for $t \in \mathbb{R}_{+}^{2}$ and $\widetilde{t} \in \mathbb{R}_{+}^{3}$, respectively, we denote the corresponding Jacobian matrices of orders $3 \times 2$ and $3 \times 3$. $\varkappa^{\prime}(t)$ coincides with $\tilde{\varkappa}^{\prime}(0, t)$ for $t \in X_{j} \subset \mathbb{R}_{+}^{2}$ if we delete the third column. Let further

$$
\Gamma_{\varkappa_{j}}(t)=\left(\operatorname{det}\left\|\left(\operatorname{grad} \varkappa_{j k}, \operatorname{grad} \varkappa_{j l}\right)\right\|_{3 \times 3}\right)^{1 / 2}
$$

denote the Gram determinant of the vector-functions grad $\varkappa_{j k} \quad(j, k=$ $1,2,3)$.

Without restricting generality we can choose the coordinates appropriately and suppose that $\tilde{\varkappa}_{j}^{\prime}(\widetilde{t})$ is an orthogonal matrix, i.e. $\left[\widetilde{\varkappa}_{j}^{\prime}(\widetilde{t})\right]^{\top}=$ $\left[\widetilde{\varkappa}_{j}^{\prime}(\widetilde{t})\right]^{-1}$. 
If the operator $V_{S}$ in (3.23) is lifted locally from the manifold $S$ to the half-space $\mathbb{R}_{+}^{2}$, we get for $t \in \mathbb{R}_{+}^{2}$ the following transformed operator

$$
\begin{aligned}
\mathbf{V}_{S, \varkappa_{j}} u(t)=\varkappa_{j *} & \mathbf{V}_{S} \varkappa_{j *}^{-1} u(t) \\
& =-\chi_{j}(t) \int_{\mathbb{R}_{+}^{2}} \mathcal{G}\left(\varkappa_{j}(t)-\varkappa_{j}(\tau)\right) \chi_{j}(\tau) \Gamma_{\varkappa_{j}}(\tau) u(\tau) \underset{(3.28)}{d \tau}
\end{aligned}
$$

where $\varkappa_{j *} \psi(t):=\chi_{j}(t) \psi\left(\varkappa_{j}(t)\right)$ and $\varkappa_{j *}^{-1} \varphi(t):=\chi_{j}^{*}(t) \varphi\left(\varkappa_{j}^{-1}(t)\right)$ with suitable smooth and compactly supported cut-off functions $\chi_{j}$ and $\chi_{j}^{*}$.

As is shown in $[1,11]$, the principal part of the pseudo-differential operator in (3.28) is given by

$$
\mathbf{V}_{S, \varkappa_{j}}^{P} u(t):=-\int_{\mathbb{R}_{+}^{2}} \mathcal{G}\left(\varkappa_{j}^{\prime}(t)(t-\tau)\right) \Gamma_{\varkappa_{j}}(t) \chi_{j}(\tau) v(\tau) d \tau
$$

for $\nu \in \mathbb{R}$ and ${ }^{8} k \geq[|\nu|]^{-}+3$. The remainder

$\mathbf{K}_{j} v(t):=-\chi_{j}(t) \int_{\mathbb{R}_{+}^{2}}\left[\mathcal{G}\left(\varkappa_{j}(t)-\varkappa_{j}(\tau)\right) \Gamma_{\varkappa_{j}}(\tau)-\mathcal{G}\left(\varkappa_{j}^{\prime}(t)(t-\tau)\right) \Gamma_{\varkappa_{j}}(t)\right] \chi_{j}(\tau) v(\tau) d \tau$

has the order -2 , i.e. the operator

$$
\mathbf{K}_{j}: \widetilde{\mathbb{H}}_{p}^{\nu}\left(\mathbb{R}_{+}^{2}\right) \rightarrow \mathbb{H}_{p}^{\nu+2}\left(\mathbb{R}_{+}^{2}\right)
$$

is bounded. Because of (3.30) and the compactness of supp $\chi_{j}$ (recall that $\chi_{j} \in C_{0}^{\infty}\left(\overline{\mathbb{R}_{+}^{2}}\right)$ ), the operator

$$
\mathbf{K}_{j}: \widetilde{\mathbb{H}}_{p}^{\nu}\left(\mathbb{R}_{+}^{2}\right) \rightarrow \mathbb{H}_{p}^{\nu+1}\left(\mathbb{R}_{+}^{2}\right)
$$

is compact (see [49]). Therefore, the symbol of $\mathbf{V}_{S, \varkappa_{j}}^{P}$, which is the principal symbol of the operator $\mathbf{V}_{S, \varkappa_{j}}$, reads as (cf. (3.14), (3.26),

\footnotetext{
${ }^{8}[s]^{-}$denotes the integer with $[s]^{-}<s \leq[s]^{-}+1$.
} 


$$
\begin{aligned}
V_{S}(x, \xi) & =-\Gamma_{\varkappa_{j}}(x) \int_{\mathbb{R}^{2}} e^{i \xi t} \mathcal{G}\left(\varkappa_{j}^{\prime}(x) t\right) d t \\
& =-\frac{\Gamma_{\varkappa_{j}}(x)}{(2 \pi)^{3}} \int_{\mathbb{R}^{2}} e^{i \xi t} \int_{\mathbb{R}^{3}} e^{-i\left[\widetilde{\varkappa}_{j}^{\prime}(x)(0, t)\right] \widetilde{y}} \mathcal{L}^{-1}(\widetilde{y}) d \widetilde{y} d t \\
& =\frac{-\Gamma_{\varkappa_{j}}(x)}{(2 \pi)^{3} \operatorname{det} \widetilde{\varkappa}_{j}^{\prime}(x)} \int_{\mathbb{R}^{2}} e^{i \xi t} \int_{\mathbb{R}^{2}} e^{-i t y} \int_{-\infty}^{\infty} \mathcal{L}^{-1}\left(\widetilde{\varkappa}_{j}^{\prime}(x) \widetilde{y}\right) d y_{0} d y d t \\
& =\frac{-\Gamma_{\varkappa_{j}}(x)}{2 \pi \operatorname{det} \widetilde{\varkappa}_{j}^{\prime}(x)} \int_{-\infty}^{\infty} \mathcal{L}^{-1}\left(\widetilde{\varkappa}_{j}^{\prime}(x)\left(\xi_{0}, \xi\right)\right) d \xi_{0} \quad \text { for } \xi \in \mathbb{R}^{2}, \quad \widetilde{y}=\left(y_{0}, y\right) \in \mathbb{R}^{3}
\end{aligned}
$$

Since $\mathcal{L}(\widetilde{\xi})=\mathcal{L}\left(\xi_{0}, \xi\right)$ is negative definite (see (3.17)), the same holds for the inverse

$$
-\left(\mathcal{L}^{-1}(\widetilde{\xi}) \eta, \eta\right)=-\left(\eta^{\prime}, \mathcal{L}(\widetilde{\xi}) \eta^{\prime}\right) \geq M_{0}\left|\eta^{\prime}\right|^{2}|\widetilde{\xi}|^{2}=M_{0}\left|\mathcal{L}^{-1}\left(|\widetilde{\xi}|^{-1} \widetilde{\xi}\right)\right||\eta|^{2}|\widetilde{\xi}|^{-2} \geq M_{1}|\eta|^{2}|\widetilde{\xi}|^{-2},
$$

too, where $\eta^{\prime}=\mathcal{L}^{-1}(\widetilde{\xi}) \eta$ and $\eta \in \mathbb{C}^{3}$. Then, with (3.31), we find

$$
\begin{aligned}
\left(V_{S}(x, \xi) \eta, \eta\right) & \geq \frac{M_{1}|\eta|^{2}}{2 \pi} \int_{-\infty}^{\infty}\left|\left(\tilde{\varkappa}_{j}^{\prime}(x)\right)^{-1}\left(\xi_{0}, \xi\right)\right|^{-2} d \xi_{0} \\
& \geq M_{2}|\eta|^{2} \int_{-\infty}^{\infty} \frac{d \xi_{0}}{\xi_{0}^{2}+|\xi|^{2}}=M|\eta|^{2}|\xi|^{-1}
\end{aligned}
$$

for all $\eta \in \mathbb{C}^{3}$ and $\xi \in \mathbb{R}^{2}$.

From (3.31) and the corresponding homogeneity properties of $\mathcal{L}(\widetilde{\xi})$ we get the homogeneity of the symbol and its derivatives,

$\partial_{x}^{\alpha} \partial_{\xi_{1}}^{m} V_{S}(x, \lambda \xi)=|\lambda|^{-1} \lambda^{-m} \partial_{x}^{\alpha} \partial_{\xi_{1}}^{m} V_{S}(x, \xi)$ for $|\alpha| \leq k-1, m \in \mathbb{N}_{0}, \xi \in \mathbb{R}^{2}, \lambda \in \mathbb{R}$.

If the local principle is applied (see e.g. $[9,13]$ ), we find that $\mathbf{V}_{S}$ in (3.23) is a Fredholm operator if and only if its local representatives

$$
\begin{gathered}
{\stackrel{\circ}{a_{S}}}_{S}\left(x_{0}, \partial\right): \mathbb{H}_{p}^{\nu}\left(\mathbb{R}^{2}\right) \rightarrow \mathbb{H}_{p}^{\nu+1}\left(\mathbb{R}^{2}\right) \quad \text { for } x_{0} \in S, \\
r_{+}{\stackrel{\circ}{a_{S}}}_{S}\left(x_{0}, \partial\right): \widetilde{\mathbb{H}}_{p}^{\nu}\left(\mathbb{R}_{+}^{2}\right) \rightarrow \mathbb{H}_{p}^{\nu+1}\left(\mathbb{R}_{+}^{2}\right) \quad \text { for } x_{0} \in \partial S
\end{gathered}
$$


with the modified symbol

$$
\begin{aligned}
\stackrel{\circ}{a}_{S}\left(x_{0}, \xi\right): & =\Gamma_{\varkappa_{j}}\left(x_{0}\right) \stackrel{\circ}{V}_{S}\left(x_{0}, \xi\right) \\
& =\Gamma_{\varkappa_{j}}\left(x_{0}\right) V_{S}\left(x_{0},\left(1+\xi_{1}^{2}\right)^{\frac{1}{2}} \operatorname{sgn} \xi_{1}, \xi_{2}\right),
\end{aligned}
$$

(cf. (2.10),(3.31)) are invertible for all $x_{0} \in \bar{S}=S \cup \partial S$ (see [13]).

Since Ker $\mathbf{V}_{S}=\{0\}$ for $\nu=-1 / 2$ and $p=2$ (see [3, 7, 25]), the solvability result follows with the help of (3.32) and (3.33) as in Theorem 2.7 .

The proposed regularity of the displacement field follows from the proved part of the theorem and from the representation (3.19) due to the embedding property

$$
\mathbb{H}_{1}^{\sim}(\mathbb{S}) \subset \mathbb{B}_{1,1}^{\sim}(\mathbb{S}) \subset \mathbb{C}^{\nu}(\mathbb{S}) \quad \text { for } \quad \nu+\frac{\not \models}{1}<\sim
$$

(see [49]) if $p<\infty$ is sufficiently large.

Lacksquare

3.3. THE NEUMANN PROBLEM. For the Neumann crack problem we need the properties of $\mathbf{D}_{S}$ defined in (3.18)(see also [2]).

Theorem 3.4 (see $[11,12]$ ). The operator

$$
\mathbf{D}_{S}: \widetilde{\mathbb{H}}_{p}^{\nu+1}(S) \rightarrow \mathbb{H}_{1}^{\nu}(\mathbb{S})
$$

is bounded for any $1<p<\infty$ and $-k+1 \leq \nu \leq k-1$.

If $k \geq 4$, then $\mathbf{D}_{S}$ in (3.38) is a Fredholm operator if and only if the conditions (3.24) hold, in which case (3.22) has a unique solution $\psi \in$ $\widetilde{\mathbb{H}}_{p}^{\nu+1}(S)$ for any given $f \in \mathbb{H}_{1}^{\nu}(\mathbb{S})$.

If $1 \leq q \leq \infty$, also the operator

$$
\mathbf{D}_{S}: \widetilde{\mathbb{B}}_{p, q}^{\nu+1}(S) \rightarrow \mathbb{B}_{1,11}^{\nu}(\mathbb{S})
$$

is bounded. If the conditions (3.24) hold, then (3.22) has a unique solution

$\varphi \in \widetilde{\mathbb{B}}_{p, q}^{\nu+1}(S)$ for any given $g \in \mathbb{B}_{1,11}^{\nu}(\mathbb{S})$.

Let $t^{ \pm} \in \bigcap_{1<p<\infty} \mathbb{H}_{1}^{-\nVdash / \nvdash}(\mathbb{S})^{9}$. Then the displacement vector $u(x)$ in (3.21) which solves the Neumann crack problem is a real analytic function in $\mathbb{R}_{S}^{3}=\mathbb{R}^{3} \backslash S$ vanishing at infinity. The traces $\gamma_{S}^{ \pm} u=u^{ \pm}$on both faces of the crack surface $S$ belong to every Hölder space $C^{\alpha}(S)$ with $0<\alpha<1 / 2$ and coincide on $\partial S: u^{-}(x)=u^{+}(x)$ for $x \in \partial S$.

\footnotetext{
${ }^{9}$ The inclusion $\left(t^{+}-t^{-}\right) \in \bigcap_{1<p<\infty} \widetilde{\mathbb{H}}_{p}^{-1 / 2}(S)$ follows since $\mathbb{H}_{1}^{-\nVdash / \not}(\mathbb{S})$ and $\widetilde{\mathbb{H}}_{p}^{-1 / 2}(S)$ can be identified for $2<p<\infty$.
} 
Proof. Let us consider equation (3.22). After the localization and local transformation of variables (similar to (3.26)-(3.35)) we get the operator representations

$$
\begin{aligned}
& \mathbf{D}_{x_{0}} v:=\chi_{j} \varkappa_{j *} D_{S} \varkappa_{j *}^{-1} \chi_{j} v=\mathcal{T}_{\varkappa_{j}} \mathbf{V}_{S}\left(x_{0}, \partial\right) \mathcal{T}_{\varkappa_{j}}^{\top} v+K_{0} v, \\
& \mathbf{D}_{x_{0}}: \mathbb{H}_{p}^{\nu+1}\left(\mathbb{R}^{2}\right) \rightarrow \mathbb{H}_{p}^{\nu}\left(\mathbb{R}^{2}\right), \\
& \left.\mathbf{K}_{x_{0}}: \mathbb{H}_{p}^{\nu+1}\left(\mathbb{R}^{2}\right) \rightarrow \mathbb{H}_{p}^{\nu+1}\left(\mathbb{R}^{2}\right) \quad \text { for } \quad x_{0} \in 3_{S}, 0\right) \\
& \mathbf{D}_{x_{0}}^{+} v:=\chi_{j} \varkappa_{j *} \mathbf{D}_{S} \varkappa_{j *}^{-1} \chi_{j} v=\mathcal{T}_{\varkappa_{j}} \mathbf{V}_{S}\left(x_{0}, \partial\right) \mathcal{T}_{\varkappa_{j}}^{\top} v+K_{0}^{+} v \text {, } \\
& \mathbf{D}_{x_{0}}^{+}: \widetilde{\mathbb{H}}_{p}^{\nu+1}\left(\mathbb{R}_{+}^{2}\right) \rightarrow \mathbb{H}_{p}^{\nu}\left(\mathbb{R}_{+}^{2}\right), \\
& \mathbf{K}_{x_{0}}^{+}: \widetilde{\mathbb{H}}_{p}^{\nu+1}\left(\mathbb{R}_{+}^{2}\right) \rightarrow \mathbb{H}_{p}^{\nu+1}\left(\mathbb{R}_{+}^{2}\right) \quad \text { for } \quad x_{0} \in\left(3.2 S^{\prime},\right.
\end{aligned}
$$

where $V_{S}\left(x_{0}, \xi\right)$ is defined in (3.31) and is positive definite (see (3.32)). Due to

$$
\left.\varkappa_{j *}(t) \operatorname{grad}_{y} \varkappa_{j *}^{-1}(t)\right|_{t=x_{0}}=\left.\frac{\partial \varkappa_{j}^{-1}(y)}{\partial y_{m}}\right|_{y=\varkappa_{j}\left(x_{0}\right)} \operatorname{grad}_{t}=\varkappa_{j}^{\prime}\left(x_{0}\right) \operatorname{grad}_{t}
$$

we get

$$
\mathcal{T}_{\varkappa_{j}}=\mathcal{N}^{\top} \mathcal{C D}\left(\varkappa_{j}^{\prime}\left(x_{0}\right) \operatorname{grad}_{t}\right) \text { with } \operatorname{grad}_{t}=\left(\partial_{t_{1}}, \partial_{t_{2}}\right)^{\top},
$$

where $\mathcal{D}(\cdot)$ is defined as the pseudo-differential operator via the symbol (3.15).

Thus the principal symbol of the operator $\mathbf{D}_{S}$ reads as

$$
\begin{gathered}
\mathbf{D}_{S}\left(x_{0}, \xi\right)=-\mathcal{T}\left(x_{0}, \xi\right) V_{S}\left(x_{0}, \xi\right) \mathcal{T}^{\top}\left(x_{0}, \xi\right), \\
\mathcal{T}\left(x_{0}, \xi\right)=\mathcal{N}^{\top} \mathcal{C} \mathcal{D}\left(\varkappa_{j}^{\prime}\left(x_{0}\right) \xi\right) \quad \text { for } \quad x_{0} \in \bar{S} \quad \text { and } \quad \xi \in \mathbb{R}_{(3 .}^{2} .
\end{gathered}
$$

Further localization with the help of Lemma 2.5 gives: $\mathbf{D}_{S}$ in (3.38) is invertible if and only if the pseudo-differential operators

$$
\begin{aligned}
& \stackrel{\circ}{b}_{S}\left(x_{0}, \partial\right): \mathbb{H}_{p}^{\nu+1}\left(\mathbb{R}^{2}\right) \rightarrow \mathbb{H}_{p}^{\nu}\left(\mathbb{R}^{2}\right), \quad \text { for } x_{0} \in S, \\
& r_{\Omega} \stackrel{\circ}{b}\left(x_{0}, \partial\right): \widetilde{\mathbb{H}}_{p}^{\nu+1}\left(\mathbb{R}_{+}^{2}\right) \rightarrow \mathbb{H}_{p}^{\nu}\left(\mathbb{R}_{+}^{2}\right) \text { for } x_{0} \in \partial S,
\end{aligned}
$$

defined by the symbol

$$
\stackrel{\circ}{b}_{S}\left(x_{0}, \xi\right):=\stackrel{\circ}{\mathcal{T}}\left(x_{0}, \xi\right) \stackrel{\circ}{a}_{S}\left(x_{0}, \xi\right) \stackrel{\circ}{\mathcal{T}}^{\top}\left(x_{0}, \xi\right),
$$

are invertible. Here $\stackrel{\circ}{a}_{S}(x, \xi)$ is defined by (3.31) and (3.36).

The symbol $D_{S}(x, \xi)$ inherits the positive definiteness from $V_{S}(x, \xi)$ in $(3.32)$, i.e.

$$
\left(D_{S}(x, \xi) \eta, \eta\right) \geq M|\xi||\eta|^{2} \quad \text { for } \quad \xi \in \mathbb{R}^{2}, \quad \eta \in \mathbb{C}^{3} \text { with } M>0 .
$$


$D_{S}(x, \xi)$ is homogeneous of order one:

$\partial_{\xi_{1}}^{m} \partial_{x}^{\alpha} D_{S}(x, \lambda \xi)=|\lambda| \lambda^{-m} \partial_{\xi_{1}}^{m} \partial_{x}^{\alpha} D_{S}\left(x_{0}, \xi\right)$ for $|\alpha| \leq k-1, m \in \mathbb{N}_{0}, \xi \in \mathbb{R}^{2}, \lambda \in \mathbb{R}$.

Since $\operatorname{Ker} \mathbf{D}_{S}=\{0\}$ for $\nu=-1 / 2$ and $p=2$ (see $\left.[3,7,25]\right)$, the solvability result follows with the help of (3.43), (3.44) as in Theorem 2.7 .

The further proof is similar to that in Theorem 3.3 and we omit the details.

Lacksquare 


\section{CRACK PROBLEMS: ASYMPTOTICS OF SOLUTIONS}

4.1. FORMULATION OF THEOREMS. Let $S \subset \mathbb{R}^{3}$ be $k$-smooth with $k \geq 4$ and $Y_{\partial S}$ be a neighbourhood of the boundary $\partial S \subset Y_{\partial S} \subset \bar{S}$ such that any $y \in Y_{\partial S}$ has only one nearest point $y_{\partial S}$ on $\partial S$. In $Y_{\partial S}$ we introduce the following local coordinates: $\rho=\rho_{\partial S}(y)=\rho\left(y, y_{\partial S}\right)$ is the Euclidean distance to $\partial S$ and $s=s(y)=s\left(y_{\partial S}\right)$ is the arc length on $\partial S$. We can suppose that the coordinate diffeomorphisms in (3.26) are given locally by $\left(x_{1}, x_{2}\right)=(s(y), \rho(y))$, where $\left(x_{1}, x_{2}\right) \in X_{j} \subset \mathbb{R}^{2}$.

Theorem 4.1. Let $u^{ \pm} \in \mathbb{H}_{1}^{\nVdash+\nVdash / 1+\sigma}(\mathbb{S})$ be given and $\left(u^{+}-u^{-}\right) \in$ $\widetilde{\mathbb{H}}_{p}^{1+1 / p+\sigma}(S),-1 / 2<\sigma<1 / 2,1<p<\infty$.

Then the solution of integral equation (3.20) has the form

$$
\varphi(s, \rho)=\rho^{-1 / 2} \chi(\rho) \varphi_{0}(s)+\varphi_{1}(s, \rho), \quad \text { for } \quad(s, \rho) \in Y_{\partial S},
$$

where $\varphi_{0} \in \mathbb{H}_{p}^{1 / 2+\sigma}(\partial S), \varphi_{1} \in \widetilde{\mathbb{H}}_{p}^{1 / p+\sigma}(S), \chi \in C_{0}^{\infty}\left(\overline{\mathbb{R}^{+}}\right)$and $\chi(\rho)=1$ if $0 \leq \rho \leq \varepsilon$ with a suitable $\varepsilon>0$.

Furthermore, we have the following a-priori estimates,

$$
\begin{aligned}
& M^{-1}\left(\left\|\varphi_{0}\left|\mathbb{H}_{p}^{1 / 2+\sigma}(\partial S)\|+\| \varphi_{1}\right| \widetilde{\mathbb{H}}_{p}^{1 / p+\sigma}(S)\right\|\right) \leq\left\|g \mid \mathbb{H}_{1}^{\nVdash+\nVdash / 1+\sigma}(\mathbb{S})\right\| \\
& \leq M\left(\left\|\left(u^{+}+u^{-}\right)\left|\mathbb{H}_{1}^{\nVdash+\nVdash / 1+\sigma}(\mathbb{S})\|+\|\left(\widetilde{\simeq}^{+}-\widetilde{\simeq}^{-}\right)\right| \widetilde{\mathbb{H}}_{1}^{\nVdash+\nVdash /+\sigma}(\mathbb{S})\right\|\right)
\end{aligned}
$$

where $g$ is given in (3.20) and $M>0$ is some constant.

If, in particular, $u^{ \pm} \in \mathbb{H}_{\infty}^{\nVdash+\sigma}(\mathbb{S})$ and $\left(u^{+}-u^{-}\right) \in \widetilde{\mathbb{H}}_{\infty}^{1+\sigma}(S)$ with $-1 / 2<\sigma<1 / 2$, then $\varphi_{0} \in C^{1 / 2+\sigma^{\prime}}(\partial S)$ with $-1 / 2<\sigma^{\prime}<\sigma$ and $\varphi_{1} \in C^{\sigma^{\prime}}(S)$ with $0<\sigma^{\prime}<\sigma$.

The theorem remains valid if the Bessel potential spaces $\mathbb{H}_{p}^{s}$ are replaced by the corresponding Besov spaces $\mathbb{B}_{p, q}^{s}$ with $1 \leq q \leq \infty$.

Theorem 4.2. Let $t^{ \pm} \in \mathbb{H}_{1}^{\nVdash / 1+\sigma}(\mathbb{S})$ be given and $\left(t^{+}-t^{-}\right) \in \widetilde{\mathbb{H}}_{p}^{1 / p+\sigma}(S)$, $-1 / 2<\sigma<1 / 2,1<p<\infty$.

Then the solution of integral equation (3.22) has the form

$$
\psi(s, \rho)=\rho^{1 / 2} \chi(\rho) \psi_{0}(s)+\psi_{1}(s, \rho), \quad \text { for } \quad(s, \rho) \in Y_{\partial S},
$$

where $\psi_{0} \in \mathbb{H}_{p}^{3 / 2+\sigma}(\partial S), \psi_{1} \in \widetilde{\mathbb{H}}_{p}^{1+1 / p+\sigma}(S), \chi \in C_{0}^{\infty}\left(\overline{\mathbb{R}^{+}}\right)$and $\chi(\rho)=1$ if $0 \leq \rho \leq \varepsilon$ with a suitable $\varepsilon>0$. 
Furthermore, we have the following a-priori estimates

$$
\begin{aligned}
& M^{-1}\left(\left\|\psi_{0}\left|\mathbb{H}_{p}^{3 / 2+\sigma}(\partial S)\|+\| \psi_{1}\right| \widetilde{\mathbb{H}}_{p}^{1+1 / p+\sigma}(S)\right\|\right) \leq\left\|f \mid \mathbb{H}_{1}^{\nVdash / 1+\sigma}(\mathbb{S})\right\| \leq \\
& \quad \leq M\left(\left\|\left(t^{+}+t^{-}\right)\left|\mathbb{H}_{1}^{\nVdash / 1+\sigma}(\mathbb{S})\|+\|\left(\approx^{+}-\approx^{-}\right)\right| \widetilde{\mathbb{H}}_{1}^{\nVdash / 1+\sigma}(\mathbb{S})\right\|\right)
\end{aligned}
$$

where $f$ is given in (3.22) and $M>0$ is some constant.

If, in particular, $t^{ \pm} \in \mathbb{H}_{\infty}^{\sigma}(\mathbb{S})$ and $\left(t^{+}-t^{-}\right) \in \widetilde{\mathbb{H}}_{\infty}^{\sigma}(S)$ with $-1 / 2<$ $\sigma<1 / 2$, then $\psi_{0} \in C^{3 / 2+\sigma^{\prime}}(\partial S)$ and $\psi_{1} \in C^{1+\sigma^{\prime}}(S)$ for any $-1 / 2<$ $\sigma^{\prime}<\sigma$.

The theorem remains valid if the Bessel potential spaces $\mathbb{H}_{p}^{s}$ are replaced by the corresponding Besov spaces $\mathbb{B}_{p, q}^{s}$ with $1 \leq q \leq \infty$.

The full asymptotics can be obtained for the following modified pseudo-differential equation on the half-space:

$$
\begin{aligned}
& \stackrel{\circ}{a}(\partial) u=v \text { on } \mathbb{R}_{+}^{n}, \\
v \in \mathbb{H}_{p}^{s-r}\left(\mathbb{R}_{+}^{n}\right), & m>\frac{n}{2}+1, \quad r, s \in \mathbb{R}, \quad 1<p<\infty,
\end{aligned}
$$

where the symbol $a \in H_{T}^{r} C^{\infty}\left(\mathbb{R}^{n}\right)$ is positive definite (cf. (2.2)) and modified according to (2.10).

Let

$$
\mathcal{H}_{p}^{\infty}\left(\mathbb{R}_{+}^{n}\right):=\bigcap_{m \geq 0} \mathbb{H}_{p}^{m}\left(\mathbb{R}_{+}^{n}\right) \text { and } \mathcal{H}_{\infty}^{\infty}\left(\mathbb{R}_{+}^{n}\right):=\bigcap_{1<p<\infty} \mathcal{H}_{p}^{\infty}\left(\mathbb{R}_{+}^{n}\right) .
$$

Then (see (1.34), (1.35))

$$
\mathcal{H}_{p}^{\infty}\left(\mathbb{R}_{+}^{n}\right)=\bigcap_{s \geq 0} \mathbb{B}_{p, q}^{s}\left(\mathbb{R}_{+}^{n}\right) \subset\left\{\varphi \in C^{\infty}\left(\overline{\mathbb{R}_{+}^{n}}\right): \varphi(\infty)=0\right\}
$$

and $\varphi \in \mathcal{H}_{p}^{\infty}\left(\mathbb{R}_{+}^{n}\right)$ implies $a(\partial) \varphi \in \mathcal{H}_{p}^{\infty}\left(\mathbb{R}_{+}^{n}\right)$ for every multiplier $a \in$ $M_{p}^{(\nu)}\left(\mathbb{R}^{n}\right)$ with any $\nu \in \mathbb{R}$ if the symbol $a\left(\xi^{\prime}, \xi_{n}+i \lambda\right)$ has an analytic extension in $\mathbb{S}^{\prime}\left(\mathbb{R}^{n-1} \times \mathbb{C}^{-}\right)$. This implication follows from (1.25) and from the footnote remark on page 14 .

We need also two classes of symbol-functions (see (1.26))

$$
\begin{gathered}
\mathbb{S}_{r}^{m}\left(\mathbb{R}^{n-1} \times \mathbb{R}\right):=\left\{a \in \mathbb{S}_{r}^{\infty, \infty}\left(\mathbb{R}^{n}, \mathbb{R}^{n-1} \times \mathbb{R}\right): a(x, \xi) \equiv a(\xi)\right\}, \\
\mathbb{S}_{r}^{m}\left(\mathbb{R}^{n-1}\right):=\left\{a \in \mathbb{S}_{r}^{\infty, \infty}\left(\mathbb{R}^{n}, \mathbb{R}^{n-1} \times \mathbb{R}\right): a\left(x, \xi^{\prime}, \xi_{n}\right) \equiv a\left(\xi^{\prime}\right)\right\}(.
\end{gathered}
$$

The class $\mathbb{S}_{r}^{\infty}\left(\mathbb{R}^{n-1}\right)$ coincides with Hörmander's class $\mathbb{S}_{1,0}^{r}\left(\mathbb{R}^{n-1}\right)$.

Theorem 4.3. Equation (4.5) has a unique solution $u \in \widetilde{\mathbb{H}}_{p}^{s}\left(\mathbb{R}_{+}^{n}\right)$ for any given $v \in \mathbb{H}_{p}^{s-r}\left(\mathbb{R}_{+}^{n}\right)$ if and only if the conditions in (2.20) hold. 
If this is the case and $v \in \mathcal{H}_{\infty}^{\infty}\left(\mathbb{R}_{+}^{n}\right)$, then the solution of Equation (4.5) has the form

$$
\begin{aligned}
& u(x)=\theta_{+}\left(x_{n}\right) e^{-x_{n}} \sum_{k=0}^{m} x_{n}^{k+r / 2} u_{k}\left(x^{\prime}\right)+x_{n}^{m+r / 2} u_{m+1}^{0}(x), \\
& \text { with } u_{k} \in \mathcal{H}_{\infty}^{\infty}\left(\mathbb{R}^{n-1}\right) \quad \text { and }\left|\partial_{x}^{\alpha} u_{m+1}^{0}(x)\right| \leq C_{\alpha} x_{n}^{\nu-\alpha_{n}}
\end{aligned}
$$

for any $\alpha \in \mathbb{N}_{0}^{n}, \quad m \in \mathbb{N}_{0}, \quad 0<\nu<1$ and some constant $C_{\alpha}>0$.

For the scalar symbol case, an expansion similar to (4.9) was obtained in $[14$, Section 7$]$ even for the general case when the symbol $a(\xi)$ has not the continuity property (2.1).

4.2. PROOF OF THEOREM 4.1. We shall apply the WienerHopf method developed in the first two sections. The method was suggested by G.Eskin in [14] for the scalar case and applied in [7] to the crack problems in an isotropic medium.

Since $g \in \mathbb{B}_{1}^{\nVdash+\nVdash / 1+\sigma}(\mathbb{S}) \subset \mathbb{H}_{1}^{\nVdash / 1+\sigma}(\mathbb{S})$, due to Theorem 3.3, Equation (3.20) has a unique solution $\varphi \in \widetilde{\mathbb{H}}_{p}^{1 / p-1+\sigma}(S)$. Let $\varkappa_{j}, \chi_{j}$ be the same as in subsection 3.2 and let $\chi_{j}^{0} \in C_{0}^{\infty}\left(\overline{\mathbb{R}_{+}^{2}}\right)$ be such that $\chi_{j}^{0} \chi_{j}=\chi_{j}^{0}$; this is possible since $\chi_{j}(x)=1$ in some neighbourhood of $X_{j}^{0} \subset X_{j} \subset \overline{\mathbb{R}_{+}^{2}}$ and $\chi_{j}^{0}(x)=1$ if $x \in X_{j}^{0}$.

After a local lifting of equation (3.20) from the neighbourhood of the boundary of the manifold $S$ to $\mathbb{R}_{+}^{2}$ we get

$$
\begin{gathered}
\chi_{j}^{0} r_{+} \mathbf{V}_{j}(x, \partial) \widetilde{\varphi}_{j}+\chi_{j}^{0} K_{j} \widetilde{\varphi}_{j}+\mathbf{T}^{1} \varphi=\chi_{j}^{0} \widetilde{g}_{j}=\widetilde{g}_{j}, \\
V_{j}(x, \xi)=\chi_{j}(x) V_{S}\left(\varkappa_{j}(x), \xi\right) \\
\widetilde{\varphi}_{j}(x)=\chi_{j}(x) \varkappa_{j *} \varphi(x)=\chi_{j}(x) \varphi\left(\varkappa_{j}(x)\right), \quad \widetilde{\varphi}_{j} \in \widetilde{\mathbb{H}}_{p}^{1 / p-1+\sigma}\left(\mathbb{R}_{+}^{2}\right), \\
\widetilde{g}_{j}(x)=\chi_{j}(x) \varkappa_{j *} g(x)=\chi_{j}(x) g\left(\varkappa_{j}(x)\right), \quad \widetilde{g}_{j} \in \mathbb{H}_{p}^{1+1 / p+\sigma}\left(\mathbb{R}_{+}^{2}\right), \\
T^{1} \varphi=\varkappa_{j *} \widetilde{\chi}_{j}^{0} V_{S}\left(1-\widetilde{\chi}_{j}\right) \varphi \in C_{0}^{k}\left(\overline{\mathbb{R}}_{+}^{2}\right), \quad \widetilde{\chi}_{j}^{0}=\varkappa_{j *}^{-1} \chi_{j}^{0}, \quad \widetilde{\chi}_{j}=\varkappa_{j *}^{-1} \chi_{j}
\end{gathered}
$$

since $\operatorname{supp} \widetilde{\chi}_{j}^{0} \cap \operatorname{supp}\left(1-\widetilde{\chi}_{j}\right)=\varnothing$. The operator $\mathbf{K}_{j}$ is defined in (3.30) and has the order -2 .

Consider the modified symbol $\stackrel{\circ}{V}_{j}(x, \xi)=V_{j}\left(x,\left(1+\xi_{1}^{2}\right)^{1 / 2} \operatorname{sgn} \xi_{1}, \xi_{2}\right)$ (cf. (2.10)). Then equation (4.10) can be rewritten in the form

$$
\begin{gathered}
\chi_{j}^{0} r_{+} \stackrel{\circ}{\mathbf{V}}_{j}(x, \partial) \widetilde{\varphi}_{j}=g_{j}^{1}, \\
g_{j}^{1}=\widetilde{g}_{j}-\chi_{j}^{0} B_{0}^{(-2)} \widetilde{\varphi}_{j}-T^{1} \varphi, \quad g_{j}^{1} \in \mathbb{H}_{p}^{1+1 / p+\sigma}\left(\mathbb{R}_{+}^{2}\right),
\end{gathered}
$$

since $\mathbf{B}_{0}^{(-2)}=\mathbf{K}_{j}+r_{+}\left[\mathbf{V}_{j}(x, \partial)-\stackrel{\circ}{\mathbf{V}}_{j}(x, \partial)\right]$ has the order -2 (see Lemma 2.5). 
Due to Theorem 2.1 and Lemma 2.3 the symbol $\stackrel{\circ}{V}_{j}(x, \xi)$ admits the factorization

$$
\stackrel{\circ}{V}_{j}(x, \xi)=\chi_{j}(x) \stackrel{\circ}{V}_{S}\left(\varkappa_{j}(x), \xi\right)=\stackrel{\circ}{a}_{-}^{-1}(x, \xi) \stackrel{\circ}{a} \stackrel{-1}{+}^{-1}(x, \xi),
$$

where $a_{-}^{ \pm 1}, a_{+}^{ \pm 1} \in H^{ \pm 1 / 2} C^{k-1, \infty}\left(\mathbb{R}_{+}^{2}, \mathbb{R}^{2}\right)$.

Due to (1.24) and (1.25) the operators

$$
\begin{aligned}
& \stackrel{\circ \pm 1}{a_{+}^{ \pm}}(x, \partial): \widetilde{\mathbb{H}}_{p}^{1 / p-1 / 2+\sigma}\left(\mathbb{R}_{+}^{2}\right) \rightarrow \widetilde{\mathbb{H}}_{p}^{1 / p-1 / 2 \mp 1 / 2+\sigma}\left(\mathbb{R}_{+}^{2}\right), \\
r_{+} & \stackrel{\circ \pm 1}{a_{-}^{ \pm 1}}(x, \partial) \ell: \mathbb{H}_{p}^{1 / p+\sigma}\left(\mathbb{R}_{+}^{2}\right) \rightarrow \mathbb{H}^{1 / p \mp 1 / 2+\sigma}\left(\mathbb{R}_{+}^{2}\right)
\end{aligned}
$$

are bounded. Since $1 / p-1<1 / p-1 / 2+\sigma<1 / p$, the spaces $\widetilde{\mathbb{H}}_{p}^{1 / p-1 / 2+\sigma}\left(\mathbb{R}_{+}^{2}\right)$ and $\mathbb{H}_{p}^{1 / p-1 / 2+\sigma}\left(\mathbb{R}_{+}^{2}\right)$ can be identified (see [49]). Thus, the operator

$$
\mathbf{B}=r_{+} \stackrel{\circ}{a}_{+}(x, \partial) r_{+} \stackrel{\circ}{a}_{-}(x, \partial) \ell: \mathbb{H}_{p}^{1 / p+\sigma}\left(\mathbb{R}_{+}^{2}\right) \rightarrow \widetilde{\mathbb{H}}_{p}^{1 / p-1+\sigma}\left(\mathbb{R}_{+}^{2}\right)
$$

is bounded. If $\mathbf{B}$ is applied to (4.11), we get with (1.24), (1.25), (1.28), (4.12) and (4.13) the local representation of the solution near the boundary,

$$
\begin{aligned}
\widetilde{\varphi}_{j}= & r_{+} \stackrel{\circ}{a}_{+}(x, \partial) r_{+} \stackrel{\circ}{a}_{-}(x, \partial) g_{j}^{1}+\varphi_{j}^{1}, \\
\varphi_{j}^{1}= & r_{+} \stackrel{\circ}{a}_{+}^{-1}(x, \partial)\left[r_{+} \stackrel{\circ}{a}_{-}^{-1}(x, \partial) r_{+} \stackrel{\circ}{a}_{-}(x, \partial)-I\right] \stackrel{\circ}{a}_{+}(x, \partial) \widetilde{\varphi}_{j} \\
& \left.+\left[I-r_{+} \stackrel{\circ}{a}_{+}^{-1}(x, \partial) r_{+} \stackrel{\circ}{a}_{+}(x, \partial)\right)\right] \widetilde{\varphi}_{j}
\end{aligned}
$$

with $\varphi_{j}^{1} \in \widetilde{\mathbb{H}}_{p}^{1 / p+\sigma}\left(\mathbb{R}_{+}^{2}\right)$, since the operators in square brackets are of order 0 .

Let us recall from [14] that

$$
\mathcal{F} \ell_{0} r_{+} \mathcal{F}^{-1} v(\xi)=\Pi_{2}^{+} v(\xi):=\frac{1}{2} v(\xi)+\frac{1}{2 \pi i} \int_{-\infty}^{\infty} \frac{v\left(\xi_{1}, \tau\right)}{\tau-\xi_{2}} d \tau
$$

where $\ell_{0}$ is the extension by 0 since $\ell_{0} r_{+} w(x)=\frac{1}{2}\left(1+\operatorname{sgn} x_{2}\right) w\left(x_{1}, x_{2}\right)$ provided $w \in L_{p}\left(\mathbb{R}^{2}\right)$; the integral is understood in the sense of a 
Cauchy v.p.. Therefore

$$
\begin{aligned}
& \left(\left[\widetilde{\lambda}_{ \pm}(\partial) \ell_{0} r_{+}-\ell_{0} r_{+} \widetilde{\lambda}_{ \pm}(\partial)\right] v, w\right)=\int_{\mathbb{R}^{2}} \mathcal{F}^{-1}\left(\widetilde{\lambda}_{ \pm} \Pi_{2}^{+}-\Pi_{2}^{+} \widetilde{\lambda}_{ \pm}\right) \mathcal{F} v(x) w(x) d x \\
& \quad=\frac{i}{(2 \pi)^{2}} \int_{-\infty}^{\infty} \int_{-\infty}^{\infty} \mathcal{F}_{\xi_{2} \rightarrow y_{2}} v\left(x_{1}, y_{2}\right) d y_{2} \int_{-\infty}^{\infty} \overline{\mathcal{F}_{x_{2} \rightarrow \xi_{2}} w\left(x_{1}, \xi_{2}\right)} d \xi_{2} d x_{1} \\
& \quad=i \int_{-\infty}^{\infty} v\left(x_{1}, 0\right) \overline{w\left(x_{1}, 0\right)} d x_{1}=i\left(\gamma_{0} v, \gamma_{0} w\right)
\end{aligned}
$$

where $\quad \gamma_{0}:=\gamma_{\mathbb{R}}, \quad \tilde{\lambda}_{ \pm}(\xi)=\xi_{2} \pm i\left(\left|\xi_{1}\right|+1\right), \quad \xi=\left(\xi_{1}, \xi_{2}\right) \in \mathbb{R}^{2}$

for any pair of test functions $v, w \in \mathbb{S}\left(\mathbb{R}^{2}\right)$.

The trace operator

$$
\gamma_{0}=\gamma_{\mathbb{R}}: \mathbb{H}_{p}^{s}\left(\mathbb{R}_{+}^{2}\right) \rightarrow \mathbb{B}_{p, p}^{s-1 / p}(\mathbb{R}) \quad \text { for } \quad s>\frac{1}{p}
$$

is bounded (see [49]); thus, (4.15) can be rewritten in the form

$$
\lambda_{ \pm}(\partial) \ell_{0} r_{+} v=\ell_{0} r_{+} \lambda_{ \pm}(\partial) v+i \gamma_{0} v \otimes \delta_{\mathbb{R}}
$$

for $v \in \mathbb{H}_{p}^{s}\left(\mathbb{R}_{+}^{2}\right)$ with $s>1 / p$. If (cf. (1.4))

$$
b_{ \pm}(x, \xi):=\lambda_{ \pm}^{-1 / 2}(\xi) \stackrel{\circ}{a_{ \pm}^{-1}}(x, \xi),
$$

then $b_{ \pm} \in \mathbb{S}_{0}^{k-1, \infty}\left(\mathbb{R}_{+}^{2}, \mathbb{R} \times \mathbb{R}\right)$ and due to Lemma 2.3 we get the representation

$$
b_{+}(x, \xi)=b_{0}(x)+b_{1}(x) \operatorname{sgn} \xi_{1}+\lambda_{0}\left(\xi_{1}\right) \lambda_{+}^{-1}(\xi) b_{2}(x, \xi)
$$

where $b_{0}, b_{1} \in C^{k-1}\left(\mathbb{R}_{+}^{2}\right)$ and $b_{2} \in \mathbb{S}_{0}^{k-1, \infty}\left(\mathbb{R}_{+}^{2}, \mathbb{R} \times \mathbb{R}\right)$.

Applying (4.11)-(4.12) and (4.17), we proceed in (4.14) by using (1.33) and obtain

$$
\begin{aligned}
\widetilde{\varphi}_{j} & =i \chi_{j}^{0}\left[\lambda_{+}^{1 / 2}(\partial) b_{+}(x, \partial) \ell_{0} r_{+} \lambda_{-}^{1 / 2}(\partial) b_{-}(x, \partial) g_{j}^{1}+\varphi_{j}^{1}+\varphi_{j}^{2}\right] \\
& =i \chi_{j}^{0}\left[e^{x_{2}} b_{+}(x, \partial) \widetilde{\lambda}_{+}^{-1 / 2}(\partial) \widetilde{\lambda}_{+}(\partial) \ell_{0} r_{+} \varphi_{j}^{0}+\varphi_{j}^{1}+\varphi_{j}^{2}+\varphi_{j}^{3}\right] \\
& =i \chi_{j}^{0}\left[e^{x_{2}} P_{+}^{-1 / 2} \gamma_{0} b_{0}\left(x, \partial_{1}\right) \varphi_{j}^{0}+\varphi_{j}^{1}+\varphi_{j}^{2}+\varphi_{j}^{3}+\varphi_{j}^{4}+\varphi_{j}^{5}\right] .
\end{aligned}
$$


Here

$$
\begin{aligned}
\varphi_{j}^{0} & =e^{-x_{2}}\left(\lambda_{-}^{1 / 2} \stackrel{\circ}{b}_{-}\right)(x, \partial) g_{j}^{1} \\
\varphi_{j}^{2} & =i \chi_{j} e^{x_{2}} \widetilde{\lambda}_{+}^{1 / 2}(\partial)\left[\widetilde{\lambda}_{+}^{-1 / 2}(\partial) \lambda_{+}^{1 / 2}(\partial)-I\right] b_{+}\left(x, \partial_{1}\right) \ell_{0} r_{+} \varphi_{j}^{0}, \\
\varphi_{j}^{3} & =\chi_{j} e^{x_{2}}\left[\widetilde{\lambda}_{+}^{1 / 2}(\partial), b_{+}(x, \partial)\right] \ell_{0} r_{+} \varphi_{j}^{0} \\
\varphi_{j}^{4} & \left.=i \chi_{j} e^{x_{2}} b_{1}(x, \partial) P_{+}^{-3 / 2}\left(I+\partial_{1}^{2}\right)^{1 / 2} \gamma_{0} \varphi_{j}^{0}\right) \\
\varphi_{j}^{5} & =\chi_{j} e^{x_{2}} b_{+}(x, \partial) \widetilde{\lambda}_{+}^{-1 / 2}(\partial)\left(\ell_{0} r_{+} \widetilde{\lambda}_{+}(\partial) \varphi_{j}^{0}\right)
\end{aligned}
$$

and $\varphi_{j}^{0} \in \mathbb{H}_{p}^{1 / p+1 / 2+\sigma}\left(\mathbb{R}_{+}^{2}\right), \varphi_{j}^{1}, \varphi_{j}^{2}, \varphi_{j}^{3}, \varphi_{j}^{4}, \varphi_{j}^{5} \in \widetilde{\mathbb{H}}_{p}^{1 / p+\sigma}\left(\mathbb{R}_{+}^{2}\right)$. The latter follows since $\ell_{0} r_{+}\left(\lambda_{-}^{1 / 2} b_{-}\right)(x, \partial) g_{j}^{1} \in \mathbb{H}_{p}^{1 / p+\sigma+1 / 2}\left(\mathbb{R}_{+}^{2}\right) \subset \mathbb{H}_{p}^{1 / p+\sigma-1 / 2}\left(\mathbb{R}_{+}^{2}\right)=$ $\widetilde{\mathbb{H}}_{p}^{1 / p+\sigma-1 / 2}\left(\mathbb{R}_{+}^{2}\right)$ and since the pseudo-differential operators in square brackets in $\varphi_{j}^{2}$ and in $\varphi_{j}^{3}$ have the orders -1 and $-1 / 2$, respectively (see Lemma 1.8). For the inclusion $\varphi_{j}^{2} \in \widetilde{\mathbb{H}}_{p}^{1 / p+\sigma}\left(\mathbb{R}_{+}^{2}\right)$ we apply also (1.24).

For $\varphi_{j}^{4}$ we have with $(4.16)\left(I+\partial_{1}^{2}\right)^{1 / 2} \gamma_{0} \varphi_{j}^{0} \in \mathbb{B}_{p, p}^{\sigma-1 / 2}(\mathbb{R})$, and $\varphi_{j}^{4} \in$ $\widetilde{\mathbb{H}}_{p}^{1 / p+\sigma}\left(\mathbb{R}_{+}^{2}\right)$ because of Lemma 1.10. Since $\ell_{0} r_{+} \stackrel{\circ}{\lambda}_{+}(x, \partial) \varphi_{j}^{0} \in \mathbb{H}_{p}^{1 / p+\sigma-1 / 2}\left(\mathbb{R}_{+}^{2}\right)=$ $\widetilde{\mathbb{H}}_{p}^{1 / p+\sigma-1 / 2}\left(\mathbb{R}_{+}^{2}\right)$, we get $\varphi_{j}^{5} \in \widetilde{\mathbb{H}}_{p}^{1 / p+\sigma}\left(\mathbb{R}_{+}^{2}\right)$ because of $(1.24)$.

Now we apply Lemma 1.12 to the first term in (4.20) with $r=1 / 2$ and obtain

$$
\begin{gathered}
i \chi_{j}^{0}(x) e^{x_{2}} P_{+}^{-1 / 2} \gamma_{0} \varphi_{j}^{0}(x)=\theta_{+} \chi_{j}^{0}(x) i c_{1 / 2}^{\prime} x_{2}^{-1 / 2} \gamma_{0} \varphi_{j}^{0}+\varphi_{j}^{6}, \\
\text { where } \quad \gamma_{0} \varphi_{j}^{0} \in \mathbb{H}_{p}^{1 / 2+\sigma}(\mathbb{R}) \quad \text { and } \quad \varphi_{j}^{6} \in \widetilde{\mathbb{H}}_{p}^{1 / p+\sigma}\left(\mathbb{R}_{+}^{2}\right) .
\end{gathered}
$$

If $\sum_{j=1}^{N} \chi_{j}^{0}(x) \equiv \chi\left(x_{2}\right)$, then (4.20)-(4.22) yield (4.1).

The right-hand side in (4.2) states that the operator $\mathbf{V}_{S}$ in (3.23) is invertible and the operator $\mathbf{U}_{S}^{0}$ in (3.18) is bounded; i.e.

$$
\begin{aligned}
& \left\|\varphi\left|\widetilde{\mathbb{H}}_{p}^{1 / p-1+\sigma}(S)\left\|\leq M_{1}\right\| g\right| \mathbb{H}_{1}^{\nVdash / 1+\sigma}(\mathbb{S})\right\| \leq \mathbb{M}_{\nVdash}\left\|ð \mid \mathbb{H}_{1}^{\nVdash+\nVdash / I+\sigma}(\mathbb{S})\right\| \\
& \leq M_{2}\left(\left\|\left(u^{+}+u^{-}\right)\left|\mathbb{H}_{1}^{\nVdash+\nVdash / 1+\sigma}(\mathbb{S})\|+\|\left(\widetilde{\simeq}^{+}-\widetilde{\simeq}^{-}\right)\right| \widetilde{\mathbb{H}}_{1}^{\nVdash+\nVdash / 1+\sigma}(\mathbb{S})\right\|\right) .
\end{aligned}
$$

Since all functions $g_{j}^{1}, \varphi_{j}^{0}, \ldots, \varphi_{j}^{6}$ in $(4.11)-(4.22)$ can be estimated by norms of $\varphi$ and $g$, we get the left-hand side of (4.2).

The inclusions $\varphi_{0} \in C^{1 / 2+\sigma^{\prime}}(\partial S), \quad \varphi_{1} \in C^{\sigma^{\prime}}(S)$ are due to the embeddings $\mathbb{B}_{p, p}^{1 / 2+\sigma}(\partial S) \hookrightarrow C^{1 / 2+\sigma^{\prime}-1 / p}(\partial S), \widetilde{\mathbb{H}}_{p}^{1 / p+\sigma}(S) \hookrightarrow C_{p}^{\sigma^{\prime}-1 / p}(S)$ for any $\sigma^{\prime}<\sigma$ if $p$ is sufficiently large.

The claim about the Besov spaces follows in the same way as in the above proof with the Bessel potential spaces, similar to the proof of Theorem 3.3. 
4.3. PROOF OF THEOREM 4.2. Here we follow the same steps as in the previous proof.

Since $f \in \mathbb{H}_{1}^{\nVdash / 1+\sigma}(\mathbb{S}) \subset \mathbb{H}_{1}^{\nVdash / 1-\nVdash+\sigma}(\mathbb{S})$, due to Theorem 3.4 equation (3.22) has an unique solution $\psi \in \widetilde{\mathbb{H}}_{p^{\prime}}^{1 / p+\sigma}(S)$. After local lifting we get, similar to (4.10), that

$$
\chi_{j}^{0} r_{+} \stackrel{\circ}{\mathbf{D}}_{j}(x, \partial) \widetilde{\psi}_{j}=f_{j}^{1} \quad \text { where } \quad f_{j}^{1} \in \mathbb{H}_{p}^{1 / p+\sigma}\left(\mathbb{R}_{+}^{2}\right) .
$$

The next step is the factorization which is possible due to (3.43), (3.44) and follows from Theorem 2.1 and Lemma 2.3:

$$
\begin{aligned}
\stackrel{\circ}{D}_{j}(x, \xi) & =\chi_{j}^{0}(x) \stackrel{\circ}{D}-\left(\varkappa_{j}(x), \xi\right) \stackrel{\circ}{D}_{+}\left(\varkappa_{j}(x), \xi\right), \\
e_{ \pm}(x, \xi) & :=\lambda_{ \pm}^{-\frac{1}{2}}(\xi) \stackrel{\circ}{D_{ \pm}^{-1}}\left(\varkappa_{j}(x), \xi\right), \text { where } e_{+} \text {can be written as } \\
e_{+}(x, \xi) & =e_{0}\left(x, \xi_{1}\right)+\left(\frac{\left(1+\xi_{1}^{2}\right)^{1 / 2}}{\xi_{2}+i\left(1+\xi_{1}^{2}\right)^{1 / 2}}\right) e_{1}(x, \xi),
\end{aligned}
$$

with $e_{0}\left(x, \xi_{1}\right)=e_{0}^{0}(x)+e_{0}^{1} \operatorname{sgn} \xi_{1}, \quad e_{ \pm}, e_{1} \in \mathbb{S}_{0}^{k-1, \infty}\left(\mathbb{R}_{+}^{2}, \mathbb{R} \times \mathbb{R}\right), \quad e_{0}^{0}, e_{0}^{1} \in C^{k-1}\left(\mathbb{R}_{+}^{2}\right)$ (cf. (4.12) and (4.18)). Similar to (4.21) we get

$$
\widetilde{\psi}_{j}=i \chi_{j}^{0}\left[e^{x_{2}} P_{+}^{-3 / 2} \gamma_{0} e_{0}\left(x, \partial_{1}\right) \psi_{j}^{0}+\psi_{j}^{1}+\psi_{j}^{2}+\psi_{j}^{3}+\psi_{j}^{4}+\psi_{j}^{5}\right]
$$

where

$$
\begin{aligned}
\psi_{j}^{0} & =e^{-x_{2}}\left(\lambda_{-}^{-1 / 2} e_{-}\right)(x, \partial) f_{j}^{1}, \\
\psi_{j}^{2} & =i \chi_{j} e^{x_{2}} \widetilde{\lambda}_{+}^{-1 / 2}(\partial)\left[\widetilde{\lambda}_{+}^{1 / 2}(\partial) \lambda_{+}^{-1 / 2}(\partial)-I\right] e_{+}\left(x, \partial_{1}\right) \ell_{0} r_{+} \psi_{j}^{0}, \\
\psi_{j}^{3} & =\chi_{j} e^{x_{2}}\left[e^{-x_{2}} \widetilde{\lambda}_{+}^{-1 / 2}(\partial), e_{+}(x, \partial)\right] \ell_{0} r_{+} \psi_{j}^{0}, \\
\psi_{j}^{4} & \left.=i \chi_{j} e^{x_{2}} e_{1}(x, \partial) P_{+}^{-5 / 2}\left(I+\partial_{1}^{2}\right)^{-1 / 2} \gamma_{0} \psi_{j}^{0}\right), \\
\psi_{j}^{5} & =\chi_{j} e^{x_{2}} e_{+}(x, \partial) \widetilde{\lambda}_{+}^{-3 / 2}(x, \partial)\left(\ell_{0} r_{+} \lambda_{+}(x, \partial) \psi_{j}^{0}\right)
\end{aligned}
$$

and $\psi_{j}^{0} \in \mathbb{H}_{p}^{1 / p+1 / 2+\sigma}\left(\mathbb{R}_{+}^{2}\right), \gamma_{0} \psi_{j}^{0} \in \mathbb{B}_{p, p}^{1 / 2+\sigma}\left(\mathbb{R}_{+}^{2}\right), \psi_{j}^{1}, \psi_{j}^{2}, \psi_{j}^{3}, \psi_{j}^{4}, \psi_{j}^{5} \in$ $\widetilde{\mathbb{H}}_{p}^{1 / p+1+\sigma}\left(\mathbb{R}_{+}^{2}\right)$.

To get the representation (4.3) we apply, similar to (4.22), Lemma 1.13 to the first term in (4.24) and find $i \chi_{j}^{0} e^{x_{2}} P_{+}^{-3 / 2} \gamma_{0} e_{0}\left(x, \partial_{1}\right) \psi_{j}^{0}=\theta_{+}\left(x_{2}\right) \chi_{j}^{0}(x) i c_{3 / 2}^{\prime} x_{2}^{1 / 2} \gamma_{0} \psi_{j}^{0}+\psi_{j}^{6}, \quad \psi_{j}^{6} \in \widetilde{\mathbb{H}}_{p}^{1+1 / p+\sigma}\left(\mathbb{R}_{+}^{2}\right)$.

The proof is completed in the same manner as in subsection 4.2. Lacksquare 
4.4. PROOF OF THEOREM 4.3. Equation (4.5) has an unique solution

$u \in \widetilde{\mathbb{H}}_{p}^{s}\left(\mathbb{R}^{n}\right)$ if and only if the lifted equation

$$
\lambda_{-}^{s-r}(\partial) \stackrel{\circ}{a}(\partial) \lambda_{+}^{-s}(\partial) u_{1}=\left(\lambda_{-}^{s-r} \stackrel{\circ}{a} \lambda_{+}^{-s}\right)(\partial) u_{1}=v_{1}
$$

with given $v_{1} \in L_{p}\left(\mathbb{R}_{+}^{n}\right)$ has an unique solution $u_{1} \in L_{p}\left(\mathbb{R}_{+}^{n}\right)$ (see (1.3),(1.4) and (1.17)). Due to Theorem 2.1 we have

$$
\lambda_{-}^{s-r}(\xi) \stackrel{\circ}{a}(\xi) \lambda_{+}^{-s}(\xi)=\left(\frac{\xi_{n}-i\left(1+\left|\xi^{\prime}\right|^{2}\right)^{1 / 2}}{\xi_{n}+i\left(1+\left|\xi^{\prime}\right|^{2}\right)^{1 / 2}}\right)^{s-\frac{r}{2}} b_{-}^{-1}(\xi) b_{+}^{-1}(\xi)
$$

where the factors $b_{+}^{ \pm 1}\left(\xi^{\prime}, \xi_{n}+i \lambda\right)$ and $b_{-}^{ \pm 1}\left(\xi^{\prime}, \xi_{n}-i \lambda\right)$ for $\lambda=0$ belong to $\mathbb{S}_{0}^{\infty}\left(\mathbb{R}^{n-1} \times \mathbb{R}\right)$ (see (4.8)) and have analytic extensions for $\lambda>0$. Then, due to the principal result in [9], the lifted operator in (4.25) is invertible in $L_{p}\left(\mathbb{R}_{+}^{n}\right)$ if and only if the inequalities

$$
\frac{1}{p}-1<s-\frac{r}{2}<\frac{1}{p}
$$

are fulfilled. These are identical with (2.20). If these inequalities hold, then the solution of (4.5) reads as

$u=\lambda_{+}^{-r / 2}(\partial) b_{+}(\partial) \ell_{0} r_{+} \lambda^{-r / 2}(\partial) b_{-}(\partial) v=\widetilde{b}_{+}(\partial) \widetilde{\lambda}_{+}^{-r / 2}(\partial) \ell_{0} r_{+} \lambda_{-}^{-r / 2}(\partial) b_{-}(\partial) v$,

where $\widetilde{b}_{+}(\xi):=\widetilde{\lambda}_{+}^{r / 2}(\xi) \lambda_{+}^{-r / 2}(\xi) b_{+}(\xi)$. The next formula is proved similar to (4.17):

$$
\ell_{0} r_{+}=\widetilde{\lambda}_{+}^{-m-1}(\partial) \ell_{0} r_{+} \widetilde{\lambda}_{+}^{m+1}(\partial)+i \sum_{k=0}^{m} P_{+}^{k-m-1} \gamma_{0} \widetilde{\lambda}_{+}^{m-k}(\partial),
$$

where $\gamma_{0}$ is the trace operator (see (3.6))

$$
\begin{aligned}
\gamma_{0}:=\gamma_{\mathbb{R}^{n-1}} & : \mathbb{H}_{p}^{s}\left(\mathbb{R}_{+}^{n}\right) \longrightarrow \mathbb{B}_{p, p}^{s-1 / p}\left(\mathbb{R}^{n-1}\right), \\
& : \mathcal{H}_{p}^{\infty}\left(\mathbb{R}_{+}^{n}\right) \longrightarrow \mathcal{H}_{p}^{\infty}\left(\mathbb{R}^{n-1}\right) \text { for } 1<p<\infty, s>\frac{1}{\left.44_{p}^{2} 9\right)}
\end{aligned}
$$

From (4.27) and (4.28) we derive the representation

$$
u=i \sum_{k=0}^{m} P_{\mathbb{R}^{n-1}}^{\tilde{\lambda}_{+}^{k-m-1-\frac{r}{2}} \widetilde{b}_{+}} \gamma_{0} \widetilde{\lambda}_{+}^{m-k}(\partial) \lambda_{-}^{-r / 2}(\partial) b_{-}(\partial) v+u_{1}^{1},
$$

where $u_{1}^{1} \in \bigcap_{1<p<\infty} \widetilde{\mathbb{H}}_{p}^{m+1 / p+\nu+r / 2}\left(\mathbb{R}_{+}^{n}\right)$ for all $0<\nu<1$. In fact, $u_{1}^{1}=\widetilde{\lambda}_{+}^{-r / 2-m-1}(\partial) \widetilde{b}_{+}(\partial) v_{1} \quad$ with $v_{45}=\ell_{0} r_{+} \widetilde{\lambda}_{+}^{m+1}(\partial) \lambda_{-}^{-r / 2}(\partial) b_{-}(\partial) v ;$ 
and the claimed inclusion in (4.30) follows from (1.24) since $v_{1} \in$ $\mathcal{H}_{\infty}^{\infty}\left(\mathbb{R}_{+}^{n}\right)$ due to $(4.7)$ while $\mathcal{H}_{\infty}^{\infty}\left(\mathbb{R}_{+}^{n}\right) \subset \mathbb{H}_{p}^{\nu-1+1 / p}\left(\mathbb{R}_{+}^{n}\right)=\widetilde{\mathbb{H}}_{p}^{\nu-1+1 / p}\left(\mathbb{R}_{+}^{n}\right)$.

Due to Lemma 2.3 we have

$$
b_{+}(\xi)=\sum_{j=0}^{k} \lambda_{0}^{j}\left(\xi^{\prime}\right) \lambda_{+}^{-j}(\xi) b_{j}\left(\xi^{\prime}\right)+\lambda_{0}^{k+1}\left(\xi^{\prime}\right) \lambda_{+}^{-k-1}(\xi) b_{k+1}^{0}(\xi)
$$

with $\quad b_{0}, \ldots, b_{k} \in \mathbb{S}_{0}^{\infty}\left(\mathbb{R}^{n-1}\right) \quad$ and $\quad b_{k+1}^{0} \in \mathbb{S}_{0}^{\infty}\left(\mathbb{R}^{n-1} \times \mathbb{R}\right),(4.31)$

where $\lambda_{0}(\xi)$ is given in (1.4) and the symbol classes are defined in (4.8). If we apply the representations

$$
\begin{gathered}
\lambda_{0}\left(\xi^{\prime}\right) \lambda_{+}^{-1}(\xi)=\sum_{j=0}^{k} \lambda_{0}^{j}\left(\xi^{\prime}\right) \widetilde{\lambda}_{+}^{-j}(\xi) d_{1, j}\left(\xi^{\prime}\right)+\lambda_{0}^{k+1}\left(\xi^{\prime}\right) \widetilde{\lambda}_{+}^{-k-1}(\xi) d_{1, k+1}^{0}(\xi) \\
\widetilde{\lambda}_{+}^{r / 2}(\xi) \lambda_{+}^{-r / 2}(\xi)=\sum_{j=0}^{k} \lambda_{0}^{j}\left(\xi^{\prime}\right) \widetilde{\lambda}_{+}^{-j}(\xi) d_{2, j}\left(\xi^{\prime}\right)+\lambda_{0}^{k+1}\left(\xi^{\prime}\right) \widetilde{\lambda}_{+}^{-k-1}(\xi) d_{2, k+1}^{0}(\xi)
\end{gathered}
$$

with $\quad d_{j, l} \in \bigcap_{1<p<\infty} M_{p}\left(\mathbb{R}^{n-1}\right) \quad$ and $\quad d_{j, k+1}^{0} \in \bigcap_{1<p<\infty} M_{p}\left(\mathbb{R}^{n}\right), \quad j=1,2, \quad l=1, \ldots, k$

where the coefficients in these representations satisfy the conditions of Theorem 1.2, then with (4.31) we get the representation

$$
\widetilde{b}_{+}(\xi)=\sum_{j=0}^{k} \lambda_{0}^{j}\left(\xi^{\prime}\right) \widetilde{\lambda}_{+}^{-j}(\xi) \widetilde{b}_{j}\left(\xi^{\prime}\right)+\lambda_{0}^{k+1}\left(\xi^{\prime}\right) \widetilde{\lambda}_{+}^{-k-1}(\xi) \widetilde{b}_{k+1}^{0}(\xi)
$$

where $\quad \widetilde{b}_{0}, \ldots, \widetilde{b}_{k} \in \bigcap_{1<p<\infty} M_{p}\left(\mathbb{R}^{n-1}\right) \quad$ and $\quad \widetilde{b}_{k+1}^{0} \in \bigcap_{1<p<\infty} M_{p}\left((\mathbb{R} ! n)^{2}\right)$

If we insert (4.32) into (4.30), apply Lemma 1.10, (4.16) and (1.37), we get

$$
\begin{aligned}
& u=i \sum_{k=0}^{m} \sum_{j=0}^{k} P_{+}^{k-m-r / 2-j-1} u_{0}^{k, j}+u_{1}^{1}+u_{2}^{1}, \\
& u_{0}^{k}=\gamma_{0} \widetilde{\lambda}_{+}^{m-k}(\partial) \lambda_{-}^{-r / 2}(\partial) b_{-}(\partial) v, \\
& u_{0}^{k, j}=\lambda_{0}^{j}\left(\partial^{\prime}\right) \widetilde{b}_{j}\left(\partial^{\prime}\right) u_{0}^{k}, \\
& u_{2}^{1}=\sum_{k=0}^{m} \widetilde{b}_{k+1}(\partial) \widetilde{\lambda}_{+}^{-m-r / 2-2}(\partial) \lambda_{0}^{k+1}\left(\partial^{\prime}\right) \widetilde{b}_{k+1}^{0}(\partial) v,
\end{aligned}
$$

where $u_{0}^{k} \in \mathcal{H}_{\infty}^{\infty}\left(\mathbb{R}^{n-1}\right)(\operatorname{see}(4.7),(4.28)) u_{0}^{k, j} \in \mathcal{H}_{\infty}^{\infty}\left(\mathbb{R}^{n-1}\right)(\operatorname{see}(1.35),(4.34))$ and $u_{2}^{1} \in \bigcap_{1<p<\infty} \widetilde{\mathbb{H}}_{p}^{m+\nu+r / 2+1}\left(\mathbb{R}_{+}^{n}\right)$ for all $0<\nu<1$. 
Thus, from (4.30) and (4.33)-(4.36) it follows

$u=i \sum_{k=0}^{m} \sum_{j=0}^{k} P_{+}^{k-m-r / 2-j-1} u_{0}^{k, j}+u_{3}^{1} \quad$ with $\quad u_{3}^{1} \in \bigcap_{1<p<\infty} \widetilde{\mathbb{H}}_{p}^{m+\nu+r / 2}\left(\mathbb{R}_{+}^{n}\right)$.

Since

$\widetilde{\mathbb{H}}_{p}^{s}\left(\mathbb{R}_{+}^{n}\right) \subset\left\{\varphi \in C^{s-n / p}\left(\mathbb{R}_{+}^{n}\right): \partial^{\alpha} \varphi\left(\xi^{\prime}, 0\right) \equiv 0\right.$ for $\left.|\alpha|<[s]-n / p\right\}$

we have

$u_{3}^{1}(x)=x_{n}^{m+r / 2} u_{3}^{2}(x)$, where $\quad\left|\partial^{\alpha} u_{3}^{2}(x)\right| \leq c_{\alpha}^{\prime} x_{n}^{\nu-\alpha_{n}} \quad$ for all $\quad \alpha \in \mathbb{N}_{0}^{n}$.

With Lemma 1.12 we get

$P_{+}^{k-m-r / 2-j-1} u_{0}^{k, j}(x)=\theta_{+}\left(x_{n}\right) e^{-x_{n}} \sum_{l=0}^{k-j} x_{n}^{m+r / 2+j-k+l} u_{0}^{k, j, l}\left(x^{\prime}\right)+x_{n}^{m+r / 2} u_{4, k, j}^{1}(x)$,

with $u_{0}^{k, j, l} \in \mathcal{H}_{\infty}^{\infty}\left(\mathbb{R}^{n-1}\right)$ and $\left|\partial_{x}^{\alpha} u_{4, k, j}^{1}(x)\right| \leq C_{\alpha}^{\prime \prime} x_{n}^{1-\alpha_{n}}$ for all $\alpha \in \mathbb{N}_{0}^{n}$.

From (4.37) now follows

$$
\begin{aligned}
u(x) & =i \theta_{+}\left(x_{n}\right) e^{-x_{n}} x_{n}^{m+r / 2} \sum_{k=0}^{m} \sum_{j=0}^{k} \sum_{l=0}^{k-j} x_{n}^{j-k+l} u_{0}^{k, j, l}\left(x^{\prime}\right)+x_{n}^{m+r / 2} u_{m+1}^{0}(x) \\
& =\theta_{+}\left(x_{n}\right) e^{-x_{n}} x_{n}^{r / 2} \sum_{k=0}^{m} x_{n}^{k} u_{k}\left(x^{\prime}\right)+x_{n}^{m+r / 2} u_{m+1}^{0}(x)
\end{aligned}
$$

where

$$
\begin{aligned}
& u_{k}=\sum_{l=k}^{m} \sum_{j=0}^{l-k} u_{0}^{l, j, l-j-k} \in \mathcal{H}_{\infty}^{\infty}\left(\mathbb{R}^{n-1}\right), \quad u_{m+1}^{0}=u_{3}^{2}+\sum_{k=0}^{m} \sum_{j=0}^{k} u_{4, k, j}^{1}, \\
& \left|\partial^{\alpha} u_{m+1}^{0}(x)\right| \leq C_{\alpha} x_{n}^{\nu-\alpha_{n}} \quad \text { for all } \quad \alpha \in \mathbb{N}_{0}^{n} \quad \text { and all } \quad 0<\nu<1 . \\
& \text { This completes the proof. } \\
& \quad
\end{aligned}
$$




\section{REFERENCES}

[1] M.Agranovich: Spectral properties of diffraction problems, pp. $279-281$. N.Voitovich, B.Kazenelenbaum, A.Sivov, Generalized Method of Eigenoscillation in the Diffraction Theory. Nauka, Moscow 1977, in Russian.

[2] E.Becache, J.Nedelec, N.Nishimura: Regularization in 3D for anisotropic elastodynamic crack and obstacle problems, Journal of Elasticity 31, 25-46 (1993).

[3] T.Buchukuri, T.Gegelia: On uniqueness of solutions of basic boundary value problems of the theory of elasticity for unbounded domains. Differencialnie Uravnenia 25, 455-479 (1989).

[4] L.Boutet de Monvel: Boundary problems for pseudo-differential operators. Acta Mathematica 126, 11-51 (1971).

[5] K.Clancey, I.Gohberg: Factorization of Matrix-Functions and Singular Integral Operators, vol. 3 of Operator Theory: Advances and Applications. Birkhäuser-Verlag, Basel 1981.

[6] M.Costabel, M.Dauge: General edge asymptotics of solutions of second order elliptic boundary value problems, I-II. Proc. Royal Soc. Edinburgh 123 A, 109-155, 157-184 (1993).

[7] M.Costabel, E.Stephan: An improved boundary element Galerkin method for three-dimensional crack problems. Integral Equations and Operator Theory 10, 467 - 504 (1987).

[8] M.Dauge: Elliptic Boundary Value Problems in Corner Domains, vol. 1341 of Lecture Notes in Mathematics. Springer-Verlag, Heidelberg 1988.

[9] R.Duduchava: On multidimensional singular integral operators, I-II. Journal of Operator Theory 11, 41-76, 199-214 (1984).

[10] R.Duduchava: Singular Integral Equations with Fixed Singularities, Teubner, Leipzig 1979.

[11] R.Duduchava, D.Natroshvili, E.Schargorodsky: On the continuity of generalized solutions of boundary value problems of the mathematical theory of cracks. Bulletin of the Georgian Academy of Sciences 135, 497-500 (1989).

[12] R.Duduchava, D.Natroshvili, E.Shargorodsky: Boundary value problems of the mathematical theory of cracks. In: Proceedings of I. Vekua Institute of Applied Mathematics, Some Problems of Elasticity Theory, vol. 39, pp. 68-84. I.Vekua Institute of Applied Mathematics, Tbilisi University. Tbilisi University Press, Tbilisi 1990.

[13] R.Duduchava, F.-O.Speck: Pseudo-differential operators on compact manifolds with Lipschitz boundary. Mathematische Nachrichten 160, 149-191 (1993).

[14] G.Eskin: Boundary Value Problems for Elliptic Pseudo-Differential Equations, vol. 52 of Translations of Mathematical Monographs. AMS, Providence, Rhode Island 1981.

[15] G.Fichera: Existence theorems in elasticity, pp. 347-389 in: Handbuch der Physik, vol. VIa/2, Festkörpermechanik II, Springer-Verlag, Berlin 1972.

[16] P.Grisvard: Elliptic Problems in Non-smooth Domains. Pitman, Boston 1985.

[17] G.Grubb: Functional Calculus of Pseudo-Differential Boundary Problems, Birkhäuser-Verlag, Boston 1986.

[18] G.Grubb: Pseudo-differential boundary problems in $L_{p}$ spaces, Communications in Partial Differential Equation, 15, 289-340 (1990). 
[19] G.Grubb, L.Hörmander:The transmission property, Mathematica Scandinavica, 67, 379-389 (1990).

[20] L.Hörmander: The Analysis of Linear Partial Differential Operators, v.I-IV. Springer-Verlag, Heidelberg 1983.

[21] G.Hsiao, E.Stephan, W.Wendland: An integral equation formulation for a boundary value problem of elasticity in the domain exterior to an arc, pp. 153-165 in: Singularities and their Constructive Treatement (P.Grisvard et al eds.), vol.1121 of Lecture Notes in Mathematics, Springer-Verlag, Heidelberg 1985.

[22] G.Hsiao, E.Stephan, W.Wendland: On the Dirichlet problem in elasticity for a domain exterior to an arc. Journal of Computational and Applied Mathematics 34, 1-19 (1991).

[23] G.Hsiao, W.Wendland: On a boundary integral method for some exterior problems in elasticity. Proc. Tbilisi University UDK 539.3, Mat.Mech.Astron. 257, 31-60 (1985).

[24] V.Kondrat'ev: Boundary problems for elliptic equations in domains with conical or angular points. Transactions Moscow Mathematical Society 16, 227-313 (1967).

[25] V.Kondrat'ev, O.Oleinik: Boundary value problems for systems of the theory of elasticity in unbounded domains. Korn's inequalities. Uspechi Matematicheskich Nauk 43, 55-98 (1988).

[26] V.Kozlov, V.Maz'ya: On stress singularities near the boundary of a polygonal crack. THD-Preprint 1289, TH Darmstadt 1990.

[27] V.Kupradze, T.Gegelia, M.Basheleishvili, T.Burchuladze: Three-Dimensional Problems of the Mathematical Theory of Elasticity and Thermoelasticity. North Holland, Amsterdam 1979.

[28] D.Kurtz: Littlewood-Paley multiplier theorems on weighted $L_{p}$-spaces. Commun. in Partial Differential Equations 16, 1263-1286 (1991).

[29] R.Leis: Initial Boundary Value Problems in Mathematical Physics. Teubner, Stuttgart 1986.

[30] G.Litvinchuk, I.Spitkovsky: Factorization of Measurable Matrix-Functions. Birkhäuser Verlag, Basel 1987.

[31] V.Maz'ya, B.Plamenevskij: On elliptic boundary value problems in a domain with piecewise smooth boundary, pp.171-181 in: Trudy Simposiuma po Mekhanike Sploshnoi Sredi i Rodstvennim Problemam Analiza I, Metsniereba, Tbilisi, 1973.

[32] V.Maz'ya and B.Plamenevskij: On a problem of Babuška (Stable asymptotics of elliptic equations of second order in domains with angular points). Mathem. Nachrichten 155, 199-220 (1992).

[33] V.Maz'ya, J.Rossmann: On the behaviour of solutions to the Dirichlet problem for second order elliptic equations near edges and polyhedral vertices with critical angles. Zeitschr. Analysis Anwendungen, to appear.

[34] S.Nazarov, B.Plamenevskij: Elliptic Problems in Domains with PiecewiseSmooth Boundaries. Nauka, Moscow 1991. In Russian.

[35] B.Noble: Methods Based on the Wiener-Hopf Technique for the Solution of Partial Differential Equations. Pergamon Press, London 1958.

[36] T.von.Petersdorff: Boundary integral equation for mixed Dirichlet, Neumann and transmission problems. Math. Meth. Appl. Sci. 11, 185 - 213 (1989). 
[37] S.Rempel, B.Schulze: Index Theory of Elliptic Boundary Value Problems. Akademie-Verlag, Berlin 1982.

[38] S.Rempel, B.Schulze: Pseudo-Differential and Mellin Operators in Spaces with Conormal Singularity. Akademie Verlag, Berlin 1987.

[39] H.Schmitz, K.Volk, W.L.Wendland: On three-dimensional singularities of elastic fields near vertices. Numerical Methods for Partial Differential Equations 9, 323-337 (1993).

[40] R.Schneider: Bessel potential operators for canonical Lipschitz domains. Mathematische Nachrichten 150, 277-299 (1991).

[41] E.Shamir: A remark on the Michlin-Hörmander multiplier theorem. Mathematical Analysis and Applications 16, 104-107 (1966).

[42] E.Shamir: Elliptic systems of singular integral equations. Transactions of the American Mathematical Society 127, 107-124 (1967).

[43] E.Shargorodsky: Boundary value problems for pseudo-differential operators on manifolds with boundary. To appear in: Trudi Tbilisskogo Matematicheskogo Instituta im. A.M.Razmadze Akademii Nauk Gruzii. In Russian. 
[44] E.Shargorodsky: Some remarks on the boundedness of pseudo-differential operators. To appear.

[45] M.Shubin: Factorization of parameter-dependent matrix-functions in normed rings and certain related questions in the theory of Noetherian operators. Mathematics USSR-Sbornik 2, 543-560 (1967).

[46] E.Stein: Singular Integrals and Differentiability Properties of Functions. Princeton Univ. Press 1970.

[47] E.Stephan: A boundary integral equation for mixed boundary value problems, screen and transmission problems in $\mathbb{R}^{\nVdash}$. THD-Preprint 848, TH Darmstadt 1984.

[48] E.Stephan, boundary

$$
\text { W.Wendland: A hypersingular }
$$
screen and crack problems. Archive Rational Mechanics and Analysis 112, 363-390 (1990).

[49] H.Triebel: Spaces,

Theory,

Function Amsterdam 1978.

[50] H.Triebel: Theory of Function Spaces. Birkhäuser-Verlag, Boston 1983.

[51] W.Wendland, J.Zhu: The boundary element method for three-dimensional Stokes flows exterior to an open surface. Mathematical and Computer Modelling 15, 19-42 (1991). 